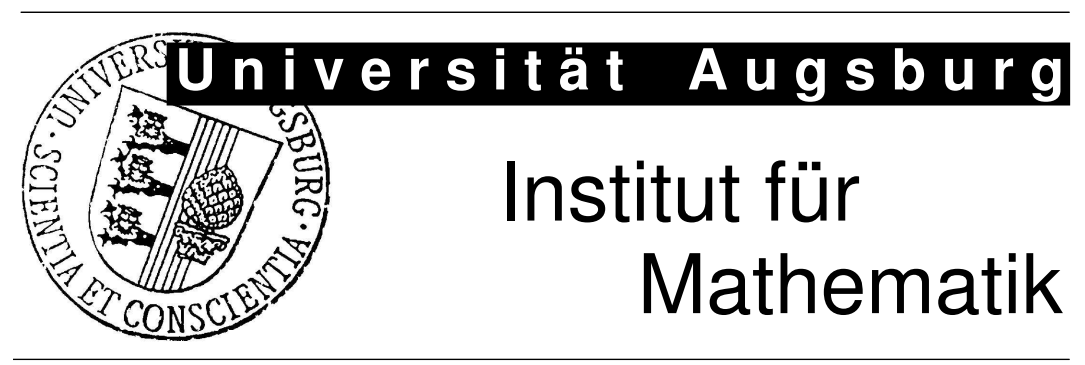

Harbir Antil, Ronald H.W. Hoppe, Christopher Linsenmann, Achim Wixforth Multiscale and Multiphysics Aspects in Modeling and Simulation of Surface Acoustic Wave Driven Microfluidic Biochips 


\section{Impressum:}

\section{Herausgeber:}

Institut für Mathematik

Universität Augsburg

86135 Augsburg

http://www.math.uni-augsburg.de/pages/de/forschung/preprints.shtml

\section{ViSdP:}

Ronald H.W. Hoppe

Institut für Mathematik

Universität Augsburg

86135 Augsburg

Preprint: Sämtliche Rechte verbleiben den Autoren (C) 2010 


\title{
Multiscale and Multiphysics Aspects in Modeling and Simulation of Surface Acoustic Wave Driven Microfluidic Biochips
}

\author{
Harbir Antil ${ }^{1}$, Ronald H.W. Hoppe ${ }^{2,3}$, Christopher Linsenmann ${ }^{3}$, and Achim Wixforth ${ }^{4}$ \\ ${ }^{1}$ Department of Computational and Applied Mathematics, Rice University, Houston, TX, USA. \\ 2 Department of Mathematics, University of Houston, Houston, TX, USA. \\ ${ }^{3}$ Institute of Mathematics, University of Augsburg, Augsburg, Germany. \\ ${ }^{4}$ Institute of Physics, University of Augsburg, Augsburg, Germany.
}

\begin{abstract}
Microfluidic biochips are devices that are designed for high throughput screening and hybridization in genomics, protein profiling in proteomics, and cell analysis in cytometry. They are used in clinical diagnostics, pharmaceutics and forensics. The biochips consist of a lithographically produced network of channels and reservoirs on top of a glass or plastic plate. The idea is to transport the injected DNA or protein probes in the amount of nanoliters along the network to a reservoir where the chemical analysis is performed. Conventional biochips use external pumps to generate the fluid flow within the network. A more precise control of the fluid flow can be achieved by piezoelectrically agitated surface acoustic waves (SAW) generated by interdigital transducers on top of the chip, traveling across the surface and entering the fluid filled channels. The fluid and SAW interaction can be described by a mathematical model which consists of a coupling of the piezoelectric equations and the compressible Navier-Stokes equations featuring processes that occur on vastly different time scales. In this contribution, we follow a homogenization approach in order to cope with the multiscale behavior of the coupled system that enables a separate treatment of the fast and slowly varying processes. The resulting model equations are the basis for the numerical simulation which is taken care of by implicit time stepping and finite element discretizations in space. Finally, the need for a better efficiency and cost effectiveness of the SAW driven biochips in the sense of a significant speed-up and more favorable reliability of the hybridization process requires an improved design which will also be addressed in this contribution. In particular, the challenge to deal with the resulting large scale optimal control and optimization problems can be met by the application of projection based model reduction techniques.
\end{abstract}

\section{INTRODUCTION}

Microfluidics is the science dealing with the behavior, precise control, and manipulation of fluids in the sub-millimeter scale. We are all familiar with the revolution brought to us by the advancement in microelectronics in our day to day life by providing, smaller, cheaper, and highly efficient devices. One should expect microfluidic sciences to follow the same path.

In life sciences, a popular concept is "labs-on-achip" which is defined as chip-based miniature laboratories that can be controlled electronically. Microfluidic biochips represent an important example (cf. Fig. (1)). The miniaturized chip laboratories are able to perform complex tasks within a few micrometers for which usually a full-size laboratory is required. Often only a very tiny amount of sample is available, e.g., in forensics and in gene ex- pression profiling analysis. Microfluidic biochips are used in pharmaceutical, medical, and forensic applications for high throughput screening, genotyping, and sequencing in genomics, protein profiling in proteomics, and cytometry in cell analysis $[88,90,101]$.

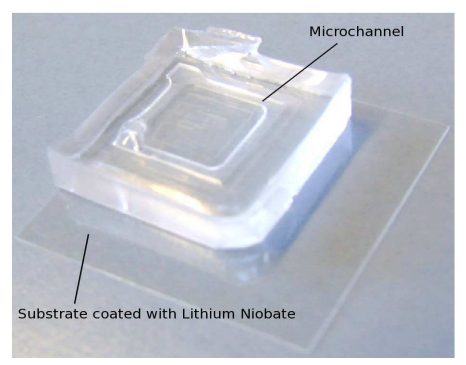

Fig. (1): Microfluidic biochip placed on a substrate 
They provide a much better sensitivity and a greater flexibility than traditional approaches. More importantly, they give rise to a significant speed-up of the hybridization processes and allow the in-situ investigation of these processes at an extremely high time resolution. This can be achieved by integrating the fluidics on top of the chip by means of a lithographically produced network of channels and reservoirs (cf. Fig. (2)).

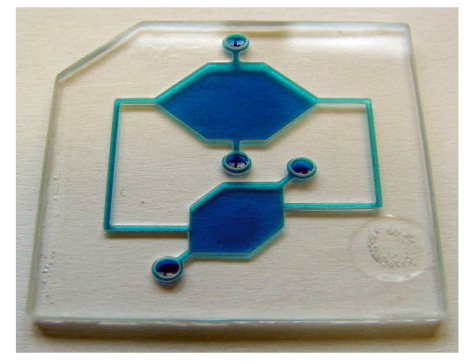

Fig. (2): Microfluidic biochip

The idea is to inject a DNA or protein containing probe and to transport it in the fluid to a reservoir where a chemical analysis is performed. The fluid flow can be taken care of by external pumps which, however, do not guarantee a very precise control of the fluid flow and are subject to wear. A new generation of biochips is based on a surface acoustic waves (SAW)-driven fluid flow [45, 56, 104, 105, 108]. Surface acoustic waves are generated by interdigital transducers (IDT), well-known from Micro-ElectroMechanical Systems (MEMS). An IDT, which is attached to a chip holder (cf. Fig (3)) is placed on top of the substrate. The chip holder holds an RFinput connection for receiving the high frequency signal produced by the signal generator. This high frequency signal (around $100 \mathrm{MHz}$ ) causes the IDT to excite and launch SAWs.

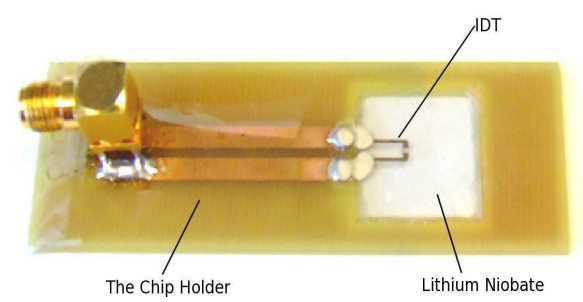

Fig. (3): Interdigital Transducer (IDT) on a substrate

The SAWs propagate through the base of the device with amplitudes in the range of nanometers and enter the fluid-filled microchannels creating sharp jets (cf. Fig. (4)). This happens within nanoseconds.

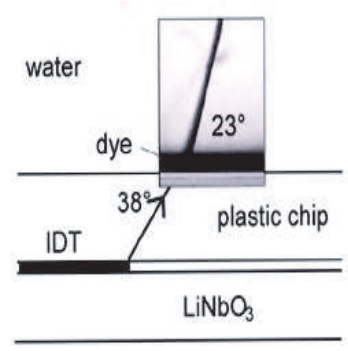

Fig. (4): Sharp jet created by surface acoustic waves

In the microchannels, the SAW get significantly damped so that an almost stationary fluid pattern emerges which is called acoustic streaming. This relaxation process occurs on a time scale of milliseconds. We are thus faced with a multiscale, multiphysics problem whose mathematical modeling and numerical simulation represents a significant challenge. The multiscale character of the problem can be appropriately taken care of by a homogenization approach. Following [3, 4, 71], after homogenization we obtain a linearized compressible NavierStokes equation and a compressible Stokes system.

Other challenging problems are various optimization issues such as the optimal design of the microchannels in order to achieve a maximum pumping rate or the design of pressure-driven capillary barriers between the channels and the reservoirs to guarantee a precise filling of the reservoirs with the probes (cf. Fig. (5)).
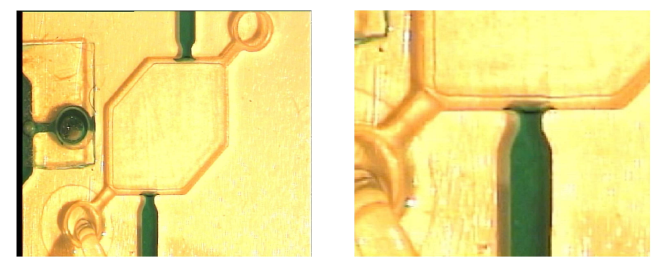

Fig. (5): Pressure-driven capillary barriers between microchannels and reservoirs

This amounts to the solution of a shape optimization problem where the mathematical model for the acoustic streaming consists of the compressible Stokes system. For the efficient solution of the optimal design problems, we have developed an adaptive multilevel interior-point method of barrier type featuring a predictor-corrector continuation method with an adaptive choice of the barrier parameter along the barrier path. The prediction step relies on a nested-iteration type tangent continuation, and the correction step is a Newton-multigrid method for the KKT system. Despite the fact that this approach 
leads to a considerable reduction in the computational work compared to more standard optimization strategies, the amount of computational time is still significant, and there is a need for further reductions. Such reductions can be achieved by model reduction based optimization methods using reduced order models for the underlying state equations generated, e.g., by Proper Orthogonal Decomposition (POD), Balanced Truncation Model Reduction (BTMR), Krylov subspace methods, or reduced basis methods (cf., e.g., [11, 13, 17, 19, 26, 27, 35, 42, $46,49,51,52,72,73,74,75,80,82,89,102,111])$.

In this contribution, we provide a survey on the mathematical modeling, analysis, numerical simulation, and optimal design of SAW driven microfluidic biochips based on recent results by the authors. In particular, in section 2 we will address in detail the multiphysics and multiscale aspects with regard to a proper modeling of the operational behavior of such biochips. Section 3 is devoted to the analysis of the model equations as given by a coupled system consisting of the linearized equations of piezoelectricity and the compressible Navier-Stokes equations, whereas section 4 deals with the development and implementation of efficient algorithmic tools for the numerical simulation $[3,4,47]$. The operational behavior can be substantially improved by optimal design. This amounts to shape optimization problems associated with the underlying acoustic streaming subproblem featuring bilateral constraints on the design variables. In section 5, we are concerned with the numerical solution of such problems using primal-dual interior-point methods by means of a path-following continuation method with an adaptive choice of the continuation parameter along the barrier path [5, 9, 10, 63]. Significant savings in computational time can be further achieved by model reduction based optimization which is realized by a combination of domain decomposition and balanced truncation $[2,6,7,8]$.

\section{Modeling}

\subsection{Piezoelectricity}

The direct piezoelectric effect in piezoelectric materials is a process characterized by the internal generation of electrical charge due to an applied mechanical force, whereas the reverse piezoelectric effect means the internal generation of a mechanical force resulting from an applied electrical field. The origin of the direct and reverse piezoelectric effect is re- lated to an asymmetry in the unit cell of a piezoelectric crystal and can be observed only in materials with a polar axis. This means that in face of a rotational symmetry around the polar axis differences in the two directions of this axis can be observed (see Fig. (6)).

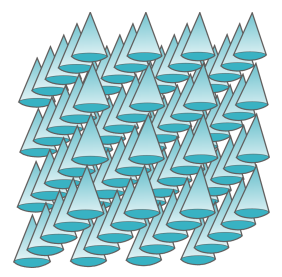

Fig. (6): Polar axis of a piezoelectric crystal

In crystallography, there are thirty-two classes of crystals. Twenty among them exhibit the piezoelectric effect. Fig. (7) (right) shows a traditional PZT (lead zirconate titanate) piezoelectric material consisting of a small, tetravalent metal ion, usually titanium or zirconium, in a lattice of larger divalent metal ions, usually lead or barium, and $\mathrm{O}_{2}$ ions. Such materials show a simple cubic symmetry above the Curie temperature and are thus isotropic before poling. After poling, they exhibit a tetragonal symmetry below the Curie temperature (see Figure (7) (right). Above this temperature, they lose the piezoelectric properties again.

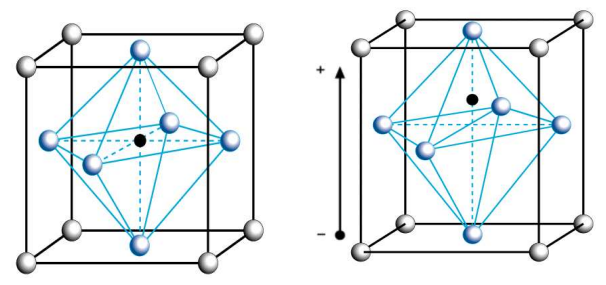

Fig. (7): Crystallographic structure of a PZT material: Temperature above (left) and below (right) the Curie point

The magnitudes of piezoelectric voltages or forces are small and often require amplification. For example, a typical disc of piezoelectric ceramic will increase or decrease in thickness by only a small fraction of a millimeter. Nevertheless, piezoelectric materials have been adapted to a wide range of applications: The direct piezoelectric effect is used in sensing applications such as force or displacement sensors. The inverse piezoelectric effect is used in actuation applications, for instance in motors and devices that precisely control positioning, and in generating sonic and ultrasonic signals. Standard 
piezoelectric materials are quartz $\left(\mathrm{SiO}_{2}\right)$, lithium niobate $\left(\mathrm{LiNbO}_{3}\right)$, or barium titanate $\left(\mathrm{BaTiO}_{3}\right)$.

In the sequel, we will consider a linear model for piezoelectricity in which the elastic, piezoelectric, and dielectric coefficients are treated as constants independent of the magnitude and the frequency of the applied mechanical stresses and the electric fields. The model is macroscopic in the sense that only mean values of the relevant physical magnitudes are incorporated. Real materials involve microscopic effects as well as mechanical and electric dissipation and nonlinear behavior. For a thorough discussion we refer to $[40,79]$ and the references therein. In piezoelectric materials, the mechanical stress $\sigma$ depends linearly on the electric field $\mathbf{E}$, in contrast to non-piezoelectric materials where the effect is quadratic.

We refer to $\Omega \subset \mathbb{R}^{d}, d=2$ or $d=3$ as a Lipschitz domain and to $[0, T] \subset \mathbb{R}_{+}$as a time interval. The mechanical displacement $\mathbf{u}=\mathbf{u}(\mathbf{x}, t)$ of a piezoelectric material of density $\rho$ in $\Omega$ that is exposed to a volume force $\mathbf{b}$ can be described by the wave equation

$$
\rho \frac{\partial^{2} \mathbf{u}}{\partial t^{2}}-\nabla \cdot \sigma=\mathbf{b} \quad \text { in } Q:=\Omega \times[0, T] .
$$

Here, $\sigma=\left(\sigma_{i j}\right)$ stands for the stress tensor which is related to the linearized strain tensor $\varepsilon(\mathbf{u})=(\nabla \mathbf{u}+$ $\left.(\nabla \mathbf{u})^{T}\right) / 2$ by the generalized Hooke's law

$$
\sigma_{i j}(\mathbf{u}, \mathbf{E})=c_{i j k l} \varepsilon_{k l}(\mathbf{u})-e_{k i j} E_{k} .
$$

Here, $\mathbf{E}$ denotes the electric field, $\mathbf{c}=\left(c_{i j k l}\right)$ is the symmetric, positive definite forth-order elasticity tensor and $\mathbf{e}=\left(e_{k i j}\right)$ refers to the symmetric thirdorder piezoelectric tensor. Note that here and in the sequel we adopt Einstein's summation convention.

In piezoelectric materials, the frequency of the occurring electric field wave is considered so small that the coupling of electromagnetic waves and elastic waves can be neglected. In other words, local perturbations in the electromagnetic field are felt almost instantaneously throughout the domain. Consequently, the electric field can be treated as quasistatic. In the model, this can be achieved by setting the magnetic permeability $\mu$ to zero which corresponds to an infinite speed of the electromagnetic wave. Maxwell's second equation then reduces to $\nabla \wedge \mathbf{E}=\mathbf{0}$. Hence, the electric field is irrotational and can be represented as the gradient of a scalar electric potential $\Phi$ according to

$$
\mathbf{E}=-\nabla \Phi
$$

The known electric field $\mathbf{E}$ determines the magnetic field $\mathbf{H}$ via Maxwell's first equation. However, in general, the magnetic field is not of interest in piezoelectric applications and is therefore not considered further. Further, piezoelectric substrates are nearly perfect insulators, i.e., the density of the free electric charges and the current density can be completely neglected. Consequently, the only relevant Maxwell equation is $\nabla \cdot \mathbf{D}=0$ with $\mathbf{D}=\mathbf{D}(\mathbf{x}, t)$ denoting the electric displacement that is related to the electric field $\mathbf{E}$ by the constitutive equation

$$
\mathbf{D}=\varepsilon \mathbf{E}+\mathbf{P} .
$$

Here, $\mathbf{P}$ is the electric polarization and $\varepsilon=\left(\varepsilon_{i j}\right)$ stands for the symmetric, positive definite permittivity tensor. In piezoelectric materials, the polarization according to external strain is linear. In analogy to the inverse effect (2), we set

$$
D_{i}(\mathbf{u}, \mathbf{E})=e_{i k l} \varepsilon_{k l}(\mathbf{u})+\varepsilon_{i j} E_{j} .
$$

Summarizing, the linear field equations of piezoelectricity are given by

$$
\begin{array}{rc}
\rho \frac{\partial^{2} u_{i}}{\partial t^{2}}-c_{i j k l} u_{k, l j}-e_{k i j} \Phi_{, k j}=b_{i} & \text { in } Q \\
e_{i k l} u_{k, l i}-\varepsilon_{i j} \Phi_{, j i}=0 & \text { in } Q
\end{array}
$$

and the constitutive equations

$$
\begin{aligned}
\sigma_{i j}(\mathbf{u}, \Phi) & =c_{i j k l} \varepsilon_{k l}(\mathbf{u})+e_{k i j} \Phi_{, k}, \\
D_{i}(\mathbf{u}, \Phi) & =e_{i k l} \varepsilon_{k l}(\mathbf{u})-\varepsilon_{i j} \Phi_{, j}
\end{aligned}
$$

The boundary $\partial \Omega$ is partitioned into two disjoint sets according to

$$
\begin{array}{llrl}
\partial \Omega & =\Gamma_{u} \cup \Gamma_{\sigma}, & \Gamma_{\sigma} & =\partial \Omega \backslash \Gamma_{u}, \\
\partial \Omega & =\Gamma_{\Phi} \cup \Gamma_{D}, & \Gamma_{D} & =\partial \Omega \backslash \Gamma_{\Phi},
\end{array}
$$

where the Dirichlet boundaries $\Gamma_{u}$ and $\Gamma_{\Phi}$ are assumed to be closed and with non-vanishing $d-1$ dimensional measure. The piezoelectric equations are supplemented by the decoupled boundary conditions

$$
\begin{aligned}
& \left.\mathbf{u}\right|_{\Gamma_{u}}=\mathbf{u}_{\Gamma},\left.\quad \sigma \cdot \mathbf{n}\right|_{\Gamma_{\sigma}}=\sigma_{n}, \\
& \left.\Phi\right|_{\Gamma_{\Phi}}=\Phi_{\Gamma},\left.\quad \mathbf{D} \cdot \mathbf{n}\right|_{\Gamma_{D}}=D_{n},
\end{aligned}
$$

and by the initial conditions

$$
\mathbf{u}(\mathbf{x}, 0)=\mathbf{u}_{0}(\mathbf{x}) \quad, \quad \frac{\partial \mathbf{u}}{\partial t}(\mathbf{x}, 0)=\mathbf{u}_{1}(\mathbf{x}) .
$$

Sometimes, it is useful to adopt a compressed notation for the piezoelectric moduli, the Voigt notation 
(see, e.g., [40, 79]). By utilizing the symmetry properties of the third- and forth-order tensors they can be reduced to higher dimensional second-order matrices. To this end, we use the identification $I=(i j)$, where

\begin{tabular}{|c||c|c|c|c|c|c|}
\hline$(i j)$ & $(11)$ & $(22)$ & $(33)$ & $(23)$ & $(13)$ & $(12)$ \\
\hline$I$ & 1 & 2 & 3 & 4 & 5 & 6 \\
\hline
\end{tabular}

i.e., $c_{I K}=c_{i j k l}, e_{i K}=e_{i k l}$ and $\varepsilon_{I}=\varepsilon_{i j}$. With this notation, the characteristic properties of a linear piezoelectric substrate are completely determined by the material matrix

$$
\begin{aligned}
& \left(\begin{array}{ll}
c_{I K} & e_{i K}^{T} \\
e_{i K} & \varepsilon_{i j}
\end{array}\right)= \\
& \left(\begin{array}{llllll}
c_{11} & c_{12} & c_{13} & c_{14} & c_{15} & c_{16} \\
c_{12} & c_{22} & c_{23} & c_{24} & c_{25} & c_{26} \\
c_{13} & c_{23} & c_{33} & c_{34} & c_{35} & c_{36} \\
c_{14} & c_{24} & c_{34} & c_{44} & c_{45} & c_{46} \\
c_{15} & c_{25} & c_{35} & c_{45} & c_{55} & c_{56} \\
c_{16} & c_{26} & c_{36} & c_{46} & c_{56} & c_{66}
\end{array}\right] \quad\left[\begin{array}{llll}
e_{11} & e_{21} & e_{31} \\
e_{12} & e_{22} & e_{32} \\
e_{13} & e_{23} & e_{33} \\
e_{14} & e_{24} & e_{34} \\
e_{15} & e_{25} & e_{35} \\
e_{16} & e_{26} & e_{36}
\end{array}\right] \\
& \left.\left[\begin{array}{llllll}
e_{11} & e_{12} & e_{13} & e_{14} & e_{15} & e_{16} \\
e_{21} & e_{22} & e_{23} & e_{24} & e_{25} & e_{26} \\
e_{31} & e_{32} & e_{33} & e_{34} & e_{35} & e_{36}
\end{array}\right] \quad\left[\begin{array}{llll}
\varepsilon_{11} & \varepsilon_{12} & \varepsilon_{13} \\
\varepsilon_{12} & \varepsilon_{22} & \varepsilon_{23} \\
\varepsilon_{13} & \varepsilon_{23} & \varepsilon_{33}
\end{array}\right]\right)
\end{aligned}
$$

where the matrices $c_{I K}$ and $\varepsilon_{i j}$ are symmetric with respect to the main diagonal. Hence, there are $21+18+6=45$ independent moduli for the most general piezoelectric substrates.

The piezoelectric material used for the SAW chip in our calculations is lithium niobate $\left(\mathrm{LiNbO}_{3}\right)$. Usually, one is interested in very large monocrystals appearing only randomly in nature. However, sophisticated production procedures for all technologically relevant materials are at hand. Depending on the cut used for the special device the monocrystals are sawed. For details concerning production procedures, natural appearances and the material constants stated here we refer to [110] and the references therein.

The material moduli are given here in a way such that the coordinate $x_{3}$-axis is identical with the polar axis $\mathbf{Z}$ along which rotatory polarization occurs (the crystallographic $\mathbf{Z}$-axis). By convention, the crystallographic axes are denoted by $\mathbf{X}, \mathbf{Y}, \mathbf{Z}$, while the coordinate axes are denoted by $x_{1}, x_{2}, x_{3}$.

Lithium niobate is an extremely versatile crystal material. It possesses a very high Curie temperature and excellent piezoelectric coupling coefficients making it attractive for ultrasonic device applications. Lithium niobate possesses a number of useful cuts that are extensively used in transducer applications, e.g. $\mathbf{Y Z ~} \mathrm{LiNbO}_{3}$ (i.e. Y-axis crystal cut, $\mathbf{Z}$-axis propagation) or $128^{\circ}$ rotated $\mathbf{Y X}$

\begin{tabular}{|c|c|c|c|c|c|c|c|}
\hline $\mathbf{c}$ & $c_{11}$ & $c_{12}$ & $c_{13}$ & $c_{14}$ & $c_{33}$ & $c_{44}$ & $c_{66}$ \\
$10^{10} \frac{\mathbf{N}}{\mathbf{m}^{2}}$ & 20.3 & 5.3 & 7.5 & 0.9 & 24.5 & 6.0 & 7.5 \\
\hline $\mathbf{e}$ & \multicolumn{2}{|c|}{$e_{15}=e_{24}$} & $e_{22}=-e_{21}$ & $e_{31}=e_{32}$ & $e_{33}$ \\
$\frac{\mathbf{C}}{\mathbf{m}^{2}}$ & \multicolumn{2}{|c|}{3.7} & 2.5 & 0.1 & 1.3 \\
\hline$\varepsilon$ & \multicolumn{3}{|c|}{$\varepsilon_{11}=\varepsilon_{22}$} & \multicolumn{2}{c|}{$\varepsilon_{33}$} \\
$10^{-12} \frac{\mathbf{F}}{\mathbf{m}}$ & \multicolumn{3}{|c|}{749.0} & 253.2 \\
\hline
\end{tabular}

Table 1: Material Moduli for $128^{\circ}$ rotated YX $\mathrm{LiNbO}_{3}$ (note that $c_{11}=c_{22}, c_{13}=c_{23}, c_{14}=-c_{24}=$ $c_{56}, c_{44}=c_{55}$ and $\left.e_{22}=-e_{16}\right)$

$\mathrm{LiNbO}_{3}$. Material moduli are given for room temperature $\left(20^{\circ} \mathrm{C}\right)$ in the following table:

The constants given here are for crystal geometries coinciding with the coordinate planes. For some technological reasons, different cuts of crystals are preferred in practice, i.e. an coordinate transformation is realized by $\underline{\bar{x}}=\underline{\mathbf{x}}(\underline{\mathrm{x}})$. The material moduli in the new coordinate system are then regained by the tensor transformations

$$
\begin{gathered}
\bar{c}_{\bar{i} \bar{j} \bar{k} \bar{l}}=c_{i j k l} \frac{\partial \bar{x}_{\bar{i}}}{\partial x_{i}} \frac{\partial x_{j}}{\partial x_{\bar{j}}} \frac{\partial \bar{x}_{\bar{k}}}{\partial x_{k}} \frac{\partial x_{l}}{\partial x_{\bar{l}}}, \\
\bar{e}_{\bar{i} \bar{j} \bar{k}}=e_{i j k} \frac{\partial \bar{x}_{\bar{i}}}{\partial x_{i}} \frac{\partial \bar{x}_{\bar{j}}}{\partial x_{j}} \frac{\partial x_{k}}{\partial x_{\bar{k}}}, \\
\bar{\varepsilon}_{\bar{i} \bar{j}}=\varepsilon_{i j} \frac{\partial \bar{x}_{\bar{i}}}{\partial x_{i}} \frac{\partial x_{j}}{\partial x_{\bar{j}}} .
\end{gathered}
$$

Usually, a simple rigid rotation is undertaken, i.e., the coordinate transformation is linear, $\underline{\bar{x}}=\underline{T} \underline{x}$, and $\frac{\partial \bar{x}_{\bar{i}}}{\partial x_{i}}=T_{\bar{i} i}$ represent the direction cosines between the two frames of reference.

In this setting, the relationship between the so-called crystallographic fundamental orthogonal system of axes $\mathbf{X}, \mathbf{Y}, \mathbf{Z}$ and the coordinate axes $x_{1}, x_{2}, x_{3}$ must be known. Note that there are piezoelectric materials where the orientations of the crystallographic unit cell axes do not align with the fundamental coordinate system, but usually constants are given for the fundamental coordinate system and we will not consider such materials anyway.

In transducer design, there is a simple standardized way [67] to provide this information: Here, the first two letters (out of $\mathbf{X}, \mathbf{Y}, \mathbf{Z}$ ) denote the initial plate orientation, the first indicating the plate thickness, the second the plate length before any rotations. The remaining three symbols $(t=$ thickness, $w=$ width, $1=$ length) are used to indicate the plate edges used for rotation, followed by a list of corresponding angles (see Fig. (8) (left) for a YZ-plate and Fig. (8) (right) for a rotated $\mathbf{Y Z} w-\phi$ plate). 


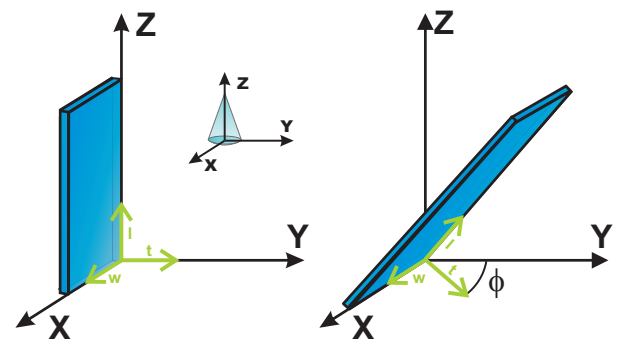

Fig. (8): Piezoelectric plate in crystallographic $\mathbf{X}, \mathbf{Y}, \mathbf{Z}$ coordinate system; $\mathbf{Y Z}$ orientation (left) and $\mathbf{Y Z} w-\phi$ orientation (right)

\subsection{Compressible Navier-Stokes Equations}

Due to the propagation of sound waves, compressible effects dominate the SAW induced fluid flow and hence, it has to be described by the compressible Navier-Stokes equations. We denote by $\Omega_{2}(t), t \in$ $\left[0, T_{2}\right]$, the time dependent domain occupied by the fluid with boundary

$$
\begin{aligned}
& \Gamma_{2}(t)=\overline{\Gamma(t)}_{2, D} \cup \overline{\Gamma(t)}_{2, N}, \\
& \Gamma_{2, D}(t) \cap \Gamma_{2, N}(t)=\emptyset,
\end{aligned}
$$

where $\Gamma_{2, D}(t)$ stands for that part of the boundary where the SAWs enter the microchannels. As a model simplification, we neglect the impact of the deflection of the walls of the microchannels on the propagation of the SAWs so that the coupling between the piezoelectric and the Navier-Stokes equations is unilateral. We denote by $v$ and $p$ the velocity and the pressure, and we refer to $\rho_{f}, \eta$, and $\xi$ as the density of the fluid and the standard and bulk viscosities. Then, the pair $(v, p)$ satisfies the following initial-boundary value problem

$$
\begin{aligned}
& \rho_{f}\left(\frac{\partial v}{\partial t}+v \cdot \nabla v\right)=\nabla \cdot \sigma, \\
& \frac{\partial \rho_{f}}{\partial t}+\nabla \cdot\left(\rho_{f} v\right)=0,
\end{aligned}
$$

in $Q_{2}:=\Omega_{2}(t) \times\left(0, T_{2}\right)$,

$$
v(\cdot+\mathbf{u}(\cdot, t), t)=\frac{\partial \mathbf{u}}{\partial t}(\cdot, t),
$$

on $\Gamma_{2, D}(t), t \in\left(0, T_{2}\right)$,

$$
\begin{aligned}
\sigma \mathbf{n} & =0 \quad \text { on } \Gamma_{2, N}(t), t \in\left(0, T_{2}\right), \\
v(\cdot, 0) & =v_{0}, \quad p(\cdot, 0)=p_{0} \quad \text { in } \Omega_{2}(0),
\end{aligned}
$$

where $\sigma=\left(\sigma_{i j}\right)_{i, j=1}^{d}$ with

$$
\sigma_{i j}:=-p \delta_{i j}+2 \eta \varepsilon_{i j}(v)+\delta_{i j}(\xi-2 \eta / 3) \nabla \cdot v
$$

in (15d). Since the deflection of the walls of the microchannels by the SAWs is approximately $10^{-9} \mathrm{~m}$ compared to lengths, widths, and heights of the microchannels in the range of $\mu \mathrm{m}$ to $\mathrm{mm}$, in the sequel we will neglect the time dependence of $\Omega_{2}$.

The SAW induced fluid flow exhibits two different time scales. When the SAWs enter the fluid filled microchannels, sharp jets and vortices are created within nanoseconds (cf. Fig. (4)). The SAWs propagate along the channels and experience a significant damping which results in a stationary flow pattern, called acoustic streaming. This relaxation process happens on a time scale of milliseconds. The multiscale character can be appropriately taken care of by a homogenization approach. Following [3, 71], we introduce a scale parameter $\varepsilon>0$ which represents the maximum deflection of the walls of the microchannels, and we consider the asymptotic expansions

$$
\begin{aligned}
\rho_{f} & =\rho_{f, 0}+\varepsilon \rho_{f}^{\prime}+\varepsilon^{2} \rho_{f}^{\prime \prime}+O\left(\varepsilon^{3}\right), \\
v & =v_{0}+\varepsilon v^{\prime}+\varepsilon^{2} v^{\prime \prime}+O\left(\varepsilon^{3}\right), \\
p & =p_{0}+\varepsilon p^{\prime}+\varepsilon^{2} p^{\prime \prime}+O\left(\varepsilon^{3}\right) .
\end{aligned}
$$

Collecting all terms of order $O(\varepsilon)$, assuming $v_{0} \equiv 0$ (fluid at rest, if no SAW actuation), and setting $\rho_{f, 1}=\varepsilon \rho_{f}^{\prime}, v_{1}:=\varepsilon v^{\prime}, p_{1}:=\varepsilon p^{\prime}$, we find that the triple $\left(\rho_{f, 1}, v_{1}, p_{1}\right)$ satisfies the linear system

$$
\begin{aligned}
\rho_{f, 0} \frac{\partial v_{1}}{\partial t}-\nabla \cdot \sigma_{1} & =\mathbf{0} & & \text { in } Q_{2}, \\
\frac{\partial \rho_{f, 1}}{\partial t}+\rho_{f, 0} \nabla \cdot v_{1} & =\mathbf{0} & & \text { in } Q_{2}, \\
v_{1} & =\mathbf{g}_{1} & & \text { on } \Gamma_{2, D}, \\
\sigma_{1} \mathbf{n} & =0 & & \text { on } \Gamma_{2, N}, \\
v_{1}(\cdot, 0)=0 \quad, \quad p_{1}(\cdot, 0) & =0 & & \text { in } \Omega_{2},
\end{aligned}
$$

where $\sigma_{1}=\left(\left(\sigma_{1}\right)_{i j}\right)_{i, j=1}^{d}, \quad\left(\sigma_{1}\right)_{i j}:=-p \quad \delta_{i j}+$ $2 \eta \varepsilon_{i j}\left(v_{1}\right)+\delta_{i j}(\xi-2 \eta / 3) \nabla \cdot v_{1}, \mathbf{g}_{1}:=\partial \mathbf{u} / \partial t$ and where $p_{1}$ and $\rho_{f, 1}$ are related by the constitutive equation

$$
p_{1}=c_{0}^{2} \rho_{f, 1} \quad \text { in } Q_{2} .
$$

Here, $c_{0}$ stands for the small signal sound speed in the fluid. The system describes the propagation and damping of the acoustic waves in the microchannels. Collecting all terms of order $O\left(\varepsilon^{2}\right)$, neglecting the time derivative with respect to the pressure, and performing the time-averaging

$$
\langle w\rangle:=T_{2}^{-1} \int_{t_{0}}^{t_{0}+T_{2}} w d t,
$$


we arrive at the compressible Stokes system

$$
\begin{aligned}
& \rho_{0} \frac{\partial v_{2}}{\partial t}-\nabla \cdot \sigma_{2}=\left\langle-\rho_{1} \frac{\partial v_{1}}{\partial t}-\rho_{0}\left(\nabla v_{1}\right) v_{1}\right\rangle, \\
& \rho_{0} \nabla \cdot v_{2}=\left\langle-\nabla \cdot\left(\rho_{1} v_{1}\right)\right\rangle, \\
& \text { in } \Omega_{2} \times(0, T] \\
& v_{2}=\mathbf{g}_{2} \quad \text { on } \Gamma_{D} \times(0, T], \\
& \sigma_{2} \mathbf{n}=0 \quad \text { on } \Gamma_{N} \times(0, T], \\
& v_{2}(\cdot, 0)=\mathbf{0}, p_{2}(\cdot, 0)=0 \quad \text { in } \Omega_{2},
\end{aligned}
$$

where $\mathbf{g}_{2}:=-\left\langle\left(\nabla v_{1}\right) \mathbf{u}\right\rangle$ in (18c) and

$$
\begin{aligned}
& \sigma_{2}=\left(\left(\sigma_{2}\right)_{i j}\right)_{i, j=1}^{2},\left(\sigma_{2}\right)_{i j}:=-p_{2} \delta_{i j} \\
& +2 \eta \varepsilon_{i j}\left(v_{2}\right)+\delta_{i j}(\xi-2 \eta / 3) \nabla \cdot v_{2} .
\end{aligned}
$$

The density $\rho_{2}$ can be obtained via the constitutive equation

$$
p_{2}=c_{0}^{2} \rho_{2} \quad \text { in } \Omega_{2} \times(0, T] .
$$

The compressible Stokes system (18a)-(18e) is used as a model for the acoustic streaming.

Remark 2.1 As a stationary version of (18a)-(18e) we may drop the time derivative $\frac{\partial v_{2}}{\partial t}$ and consider the Stokes system

$$
\begin{aligned}
-\nabla \cdot \sigma_{2} & =\left\langle-\rho_{1} \frac{\partial v_{1}}{\partial t}-\rho_{0}\left(\nabla v_{1}\right) v_{1}\right\rangle \\
\rho_{0} \nabla \cdot v_{2} & =\left\langle-\nabla \cdot\left(\rho_{1} v_{1}\right)\right\rangle
\end{aligned}
$$

in $\Omega_{2} \times(0, T]$,

$$
\begin{aligned}
v_{2} & =\mathbf{g}_{2} \quad \text { on } \Gamma_{D} \times(0, T], \\
\sigma_{2} \mathbf{n} & =0 \quad \text { on } \Gamma_{N} \times(0, T] .
\end{aligned}
$$

\begin{tabular}{|c|c|c|c|}
\hline$\rho_{f}\left(\mathrm{~kg} / \mathrm{m}^{3}\right)$ & $\eta(\mathrm{kg} /(\mathrm{ms}))$ & $\xi(\mathrm{kg} /(\mathrm{ms}))$ & $c_{0}(\mathrm{~m} / \mathrm{s})$ \\
\hline $1.0 \cdot 10^{3}$ & $1.002 \cdot 10^{-4}$ & $7.97 \cdot 10^{-4}$ & $1.484 \cdot 10^{3}$ \\
\hline
\end{tabular}

Table 2: Microfluidic material data (water at $20^{\circ}$ )

Table 2 contains relevant physical parameters for the acoustic streaming problem.

\section{Analysis}

In the sequel, we adopt standard notation of Lebesgue and Sobolev space theory (cf., e.g.,
[95]). We denote by $L^{2}(\Omega)\left(\mathbf{L}^{2}(\Omega)\right)$ the Lebesgue space of square integrable complex valued functions (vector fields) on $\Omega$ with inner product $(\cdot, \cdot)_{0, \Omega}$ and norm $\|\cdot\|_{0, \Omega}$ and by $H^{k}(\Omega)\left(\mathbf{H}^{k}(\Omega)\right)$ the Sobolev space of complex valued square integrable functions (vector fields) having square integrable weak derivatives up to order $k \in \mathbb{N}$ with inner product $(\cdot, \cdot)_{k, \Omega}$ and norm $\|\cdot\|_{k, \Omega}$. For $\Gamma^{\prime} \subseteq$ $\partial \Omega$, we refer to $H^{1 / 2}\left(\Gamma^{\prime}\right)\left(\mathbf{H}^{1 / 2}\left(\Gamma^{\prime}\right)\right)$ as the trace space associated with $H^{1}(\Omega)\left(\mathbf{H}^{1}(\Omega)\right)$. The subspace $H_{0, \Gamma^{\prime}}^{1}(\Omega)\left(\mathbf{H}_{0, \Gamma^{\prime}}^{1}(\Omega)\right)$ stands for the subspace of functions (vector fields) on $\Omega$ with vanishing trace on $\Gamma^{\prime}$ (omitting the subindex $\Gamma^{\prime}$, if $\Gamma^{\prime}=$ $\partial \Omega)$. Moreover, we denote by $H_{00}^{1 / 2}\left(\Gamma^{\prime}\right) \subset H^{1 / 2}\left(\Gamma^{\prime}\right)$ $\left(\mathbf{H}_{00}^{1 / 2}(\Omega) \subset \mathbf{H}^{1 / 2}\left(\Gamma^{\prime}\right)\right)$ the subspace of functions (vector fields) whose extension by zero to all of $\partial \Omega$ belongs to $H^{1 / 2}(\partial \Omega)\left(\mathbf{H}^{1 / 2}(\partial \Omega)\right)$ and defines a bounded linear operator. The associated dual spaces are referred to as $H^{-1 / 2}\left(\Gamma^{\prime}\right)\left(\mathbf{H}^{-1 / 2}\left(\Gamma^{\prime}\right)\right)$.

For the ease of notation, we set $\mathbf{V}:=\mathbf{H}_{0, \Gamma_{u}}^{1}(\Omega), W:=$ $H_{0, \Gamma_{\Phi}}^{1}(\Omega)$ and denote by $\mathbf{V}^{*}$ and $W^{*}$ the associated dual spaces.

\subsection{Piezoelectric Equations}

The SAWs are usually excited by an interdigital transducer located at $\Gamma_{\Phi}$ which operates at some fixed frequency $\omega>0$. The excitation is treated as a Dirichlet boundary condition for the electric potential $\Phi$. Under the assumption that there is no further volume force $\mathbf{b}$, the piezoelectric equations reduce to

$$
\begin{aligned}
\rho \frac{\partial^{2} u_{i}}{\partial t^{2}}-c_{i j k l} \frac{\partial^{2} u_{k}}{\partial x_{l} \partial x_{j}}-e_{k i j} \frac{\partial^{2} \Phi}{\partial x_{k} \partial x_{j}} & =0 \\
e_{i k l} \frac{\partial^{2} u_{k}}{\partial x_{l} \partial x_{i}}-\varepsilon_{i j} \frac{\partial^{2} \Phi}{\partial x_{j} \partial x_{i}} & =0
\end{aligned}
$$

We are interested in time-harmonic solutions

$$
\begin{aligned}
\mathbf{u}(\mathbf{x}, t) & =\operatorname{Re}(\mathbf{u}(\mathbf{x}) \exp (-\beta \omega t)) \\
\Phi(\mathbf{x}, t) & =\operatorname{Re}(\Phi(\mathbf{x}) \exp (-\beta \omega t))
\end{aligned}
$$

with complex valued functions $\mathbf{u}$ and $\Phi$. The elastic and electric Dirichlet and Neumann boundary data are given according to

$$
\begin{array}{ll}
\mathbf{u}_{\Gamma} \in \mathbf{H}^{1 / 2}\left(\Gamma_{u}\right), & \Phi_{\Gamma} \in H^{1 / 2}\left(\Gamma_{\Phi}\right), \\
\sigma_{n} \in \mathbf{H}^{-\frac{1}{2}}\left(\Gamma_{\sigma}\right), & D_{n} \in H^{-\frac{1}{2}}\left(\Gamma_{D}\right) .
\end{array}
$$

We introduce the subspaces

$$
\begin{gathered}
\mathbf{V}_{\Gamma_{u}}:=\mathbf{V}+\mathbf{E}_{\Gamma_{u}}\left(\mathbf{u}_{\Gamma}\right), \\
W_{\Gamma_{\Phi}}:=W+E_{\Gamma_{\Phi}}\left(\Phi_{\Gamma}\right),
\end{gathered}
$$


where

$$
\begin{aligned}
& \mathbf{E}_{\Gamma_{u}}: \mathbf{H}^{1 / 2}\left(\Gamma_{u}\right) \rightarrow \mathbf{H}^{1}(\Omega), \\
& E_{\Gamma_{\Phi}}: H^{1 / 2}\left(\Gamma_{\Phi}\right) \rightarrow H^{1}(\Omega)
\end{aligned}
$$

are uniquely defined extension operators, and we refer to $\mathbf{R}_{\Gamma_{\sigma}}: \mathbf{H}^{1}(\Omega) \rightarrow \mathbf{H}^{-1 / 2}\left(\Gamma_{\sigma}\right)$ and $R_{\Gamma_{D}}$ : $H^{1}(\Omega) \rightarrow H^{-1 / 2}\left(\Gamma_{D}\right)$ as trace operators.

The variational formulation of the problem of piezoelectrically actuated SAWs then reads: Find $\mathbf{u} \in \mathbf{V}_{\Gamma_{u}}$ and $\Phi \in W_{\Gamma_{\Phi}}$ such that for all $\mathbf{v} \in \mathbf{V}$ and $\psi \in W$

$$
\begin{aligned}
a(\mathbf{u}, \mathbf{v})+b(\Phi, \mathbf{v}) & \\
-\omega^{2}(\mathbf{u}, \mathbf{v})_{0, \Omega} & =<\sigma_{n}, \mathbf{v}> \\
b(\psi, \mathbf{u})-c(\Phi, \psi) & =<D_{n}, \psi>
\end{aligned}
$$

Here, $a, b$ and $c$ stand for the sesquilinear forms

$$
\begin{aligned}
a(\mathbf{v}, \mathbf{w}) & :=\int_{\Omega} c_{i j k l} \varepsilon_{k l}(\mathbf{v}) \varepsilon_{i j}(\overline{\mathbf{w}}) d \mathbf{x} \\
b(\varphi, \mathbf{v}) & :=\int_{\Omega} e_{k i j} \frac{\partial \varphi}{\partial x_{k}} \varepsilon_{i j}(\overline{\mathbf{v}}) d \mathbf{x} \\
c(\varphi, \psi) & :=\int_{\Omega} \varepsilon_{i j} \frac{\partial \varphi}{\partial x_{i}} \frac{\partial \bar{\psi}}{\partial x_{j}} d \mathbf{x},
\end{aligned}
$$

where $\mathbf{v}, \mathbf{w} \in \mathbf{H}^{1}(\Omega), \varphi, \psi \in H^{1}(\Omega)$ and $\overline{\mathbf{w}}, \bar{\psi}$ denotes complex conjugation. Moreover, $\langle\cdot, \cdot\rangle$ refers both to the dual pairing between $\mathbf{H}^{-1 / 2}\left(\Gamma_{\sigma}\right)$ and $\mathbf{H}_{00}^{1 / 2}\left(\Gamma_{\sigma}\right)$ and to the dual pairing between $H^{-1 / 2}\left(\Gamma_{D}\right)$ and $H_{00}^{1 / 2}\left(\Gamma_{D}\right)$.

The above sesquilinear forms define linear operators $\mathbf{A}: \mathbf{H}^{1}(\Omega) \rightarrow \mathbf{V}^{*}, \mathbf{B}: H^{1}(\Omega) \rightarrow \mathbf{V}^{*}$ and $\mathbf{C}: H^{1}(\Omega) \rightarrow$ $W^{*}$ so that the operator theoretic form of (26a),(26b) is given by: Find $\mathbf{u} \in \mathbf{V}$ and $\Phi \in W$ such that

$$
\begin{aligned}
& \left(\mathbf{A}-\omega^{2} \mathbf{I}\right) \mathbf{u}+\mathbf{B} \Phi=\mathbf{f} \\
& \mathbf{B}^{*} \mathbf{u}-\mathbf{C} \Phi \\
& =g .
\end{aligned}
$$

Here, I stands for the injection $\mathbf{I}: \mathbf{V} \rightarrow \mathbf{V}^{*}$, and the right-hand sides $\mathbf{f} \in \mathbf{V}^{*}, g \in W^{*}$ are given according to

$$
\begin{aligned}
\mathbf{f} & :=\mathbf{R}_{\Gamma_{\sigma}}^{*}\left(\sigma_{n}\right)-\left(\mathbf{A}-\omega^{2} \mathbf{I}\right) \mathbf{E}_{\Gamma_{u}}\left(\mathbf{u}_{\Gamma}\right)-\mathbf{B} E_{\Gamma_{\Phi}}\left(\Phi_{\Gamma}\right), \\
g & :=R_{\Gamma_{D}}^{*}\left(D_{n}\right)-\mathbf{B}^{*} \mathbf{E}_{\Gamma_{u}}\left(\mathbf{u}_{\Gamma}\right)+\mathbf{C} E_{\Gamma_{\Phi}}\left(\Phi_{\Gamma}\right) .
\end{aligned}
$$

Lemma 3.1 The operators $\mathbf{A}, \mathbf{B}$ and $\mathbf{C}$ are bounded linear operators. Moreover, the operator $\mathbf{A}$ is symmetric and $\mathbf{V}$-elliptic, and the operator $\mathbf{C}$ is symmetric and $W$-elliptic.

Proof: The continuity of $\mathbf{A}, \mathbf{B}$ and $\mathbf{C}$ is obvious. The symmetry of $\mathbf{A}$ follows from the symmetry of the elasticity tensor $\mathbf{c}$, and the $\mathbf{V}$-ellipticity of $\mathbf{A}$ is a direct consequence of the positive definiteness of c and Korn's inequality. Likewise, the symmetry of C follows from the symmetry of the piezoelectric tensor $\varepsilon$ and the $W$-ellipticity can be deduced from the positive definiteness of $\varepsilon$.

Elimination of $\Phi$ from (28a),(28b) results in the Schur complement system

$$
\mathbf{S u}-\omega^{2} \mathbf{I u}=\mathbf{F},
$$

where the Schur complement $\mathbf{S}: \mathbf{V} \rightarrow \mathbf{V}^{*}$ and the right-hand side $\mathbf{F}$ are given by

$$
\mathbf{S}:=\mathbf{A}+\mathbf{B C}^{-1} \mathbf{B}^{*}
$$

and

$$
\mathbf{F}:=\mathbf{f}+\mathbf{B C}^{-1} g .
$$

Lemma 3.2 The Schur complement $\mathbf{S}$ is a bounded, symmetric and $\mathbf{V}$-elliptic linear operator. For the the norm $\|\mathbf{S}\|$ and the ellipticity constant $\gamma_{S}$ of $\mathbf{S}$ we have the estimates

$$
\|\mathbf{S}\| \leq\|\mathbf{A}\|+\frac{\|\mathbf{B}\|^{2}}{\gamma_{C}} \quad, \quad \gamma_{S} \geq \gamma_{A}
$$

where $\gamma_{A}$ and $\gamma_{C}$ are the ellipticity constants of $\mathbf{A}$ and $\mathbf{C}$, respectively.

Proof: The symmetry of $\mathbf{S}$ is obvious. For $\mathbf{v}, \mathbf{w} \in \mathbf{V}$ we have

$$
\begin{aligned}
& <\mathbf{S v}, \mathbf{w}>=<\mathbf{A v}, \mathbf{w}>+<\mathbf{C}^{-1} \mathbf{B}^{*} \mathbf{v}, \mathbf{B}^{*} \mathbf{w}> \\
& \leq\left(\|\mathbf{A}\|+\left\|\mathbf{C}^{-1}\right\|\|\mathbf{B}\|^{2}\right)\|\mathbf{v}\|_{1, \Omega}\|\mathbf{w}\|_{1, \Omega} .
\end{aligned}
$$

Hence, taking $\left\|\mathbf{C}^{-1}\right\| \leq \gamma_{C}^{-1}$ into account, this gives the upper bound for $\|\mathbf{S}\|$ in (33). The lower bound for $\gamma_{S}$ can be readily deduced from

$$
\begin{aligned}
& <\mathbf{S v}, \mathbf{v}>=<\mathbf{A v}, \mathbf{v}> \\
& +<\mathbf{C}^{-1} \mathbf{B}^{*} \mathbf{v}, \mathbf{B}^{*} \mathbf{v}>\geq \gamma_{A}\|\mathbf{v}\|_{1, \Omega}^{2}
\end{aligned}
$$

By introducing the operator $\mathbf{S}_{R}^{-1}: \mathbf{L}^{2}(\Omega) \rightarrow \mathbf{V} \subset$ $\mathbf{L}^{2}(\Omega)$ according to

$$
\mathbf{S}_{R}^{-1} \mathbf{v}:=\mathbf{S}^{-1} \mathbf{v}, \quad \mathbf{v} \in \mathbf{L}^{2}(\Omega),
$$

and rewriting (30) as

$$
\mathbf{S u}-\omega^{2} \mathbf{u}=-\omega^{2} \mathbf{S}\left(\mathbf{S}_{R}^{-1}-\omega^{-2}\right) \mathbf{u}=\mathbf{F},
$$

it can be shown that the following Fredholm alternative holds true. 
Theorem 3.3 a) For $\omega^{2} \in \mathbb{R}$ exactly one of the following alternatives holds true:

(i) $\mathbf{u}=\mathbf{0}$ is the only solution of the eigenvalue problem $\mathbf{S u}=\omega^{2} \mathbf{I u}$. In this case, for every $\mathbf{F} \in \mathbf{V}^{*}$ the equation $\left(\mathbf{S}-\omega^{2} \mathbf{I}\right) \mathbf{u}=\mathbf{F}$ admits a unique solution $\mathbf{u} \in \mathbf{V}$ depending continuously on $\mathbf{F}$.

(ii) There is a finite number $M$ of linearly independent eigenfunctions $\mathbf{u}_{1}, \ldots \mathbf{u}_{M}$ satisfying $\mathbf{S u}_{m}=$ $\omega^{2} \mathbf{I} \mathbf{u}_{m}$. In this case, if $\overline{\mathbf{u}}$ solves $\left(\mathbf{S}-\omega^{2} \mathbf{I}\right) \mathbf{u}=\mathbf{F}$ (i.e., if the equation is solvable), the general solution can be obtained with arbitrary $\alpha_{m} \in \mathbb{R}$ by

$$
\mathbf{u}=\overline{\mathbf{u}}+\sum_{m=1}^{M} \alpha_{m} \mathbf{u}_{m} .
$$

b) The spectrum of $\mathbf{S}$ consists of a sequence of countably many real eigenvalues $0<\omega_{1}^{2}<\omega_{2}^{2}<\ldots$ tending to infinity, i.e., $\lim _{j \rightarrow \infty} \omega_{j}^{2}=\infty$.

c) If $\omega^{2} \in \mathbb{R}$ is an eigenvalue of $\mathbf{S}$, the equation $\left(\mathbf{S}-\omega^{2} \mathbf{I}\right) \mathbf{u}=\mathbf{F}$ is solvable if and only if $\mathbf{F} \in(\mathbf{S}-$ $\left.\omega^{2} \mathbf{I}\right)(\mathbf{V})$, i.e., iff $\mathbf{F} \in \operatorname{Ker}\left(\mathbf{S}-\omega^{2} \mathbf{I}\right)^{0}$ where

$$
\begin{aligned}
& \operatorname{Ker}\left(\mathbf{S}-\omega^{2} \mathbf{I}\right)^{0}:= \\
& \left\{\mathbf{v}^{*} \in \mathbf{V}^{*} \mid<\mathbf{v}^{*}, \mathbf{v}>=0, \mathbf{v} \in \operatorname{Ker}\left(\mathbf{S}-\omega^{2} \mathbf{I}\right)\right\} .
\end{aligned}
$$

Proof: The operator $\mathbf{S}_{R}^{-1}$ (cf. (34)) is symmetric in $\mathbf{L}^{2}(\Omega)$ which follows easily from the symmetry of S. It is bounded, since for $\mathbf{v} \in \mathbf{L}^{2}(\Omega)$

$$
\begin{aligned}
& \gamma_{S}\left\|\mathbf{S}_{R}^{-1} \mathbf{v}\right\|_{0, \Omega}^{2} \leq \gamma_{S}\left\|\mathbf{S}_{R}^{-1} \mathbf{v}\right\|_{1, \Omega}^{2} \\
& \leq<\mathbf{S S}_{R}^{-1} \mathbf{v}, \mathbf{S}_{R}^{-1} \mathbf{v}>=\left(\mathbf{v}, \mathbf{S}_{R}^{-1} \mathbf{v}\right)_{0, \Omega} \\
& \leq\|\mathbf{v}\|_{0, \Omega}\left\|\mathbf{S}_{R}^{-1} \mathbf{v}\right\|_{0, \Omega} .
\end{aligned}
$$

Moreover, for a generalized eigenvalue $\omega^{2} \neq 0$ and a corresponding eigenfunction $\mathbf{u} \in \mathbf{V}$ of $\mathbf{S}$, the operator $\mathbf{S}_{R}^{-1}$ satisfies the inverse eigenvalue problem

$$
\mathbf{S}_{R}^{-1} \mathbf{u}=\frac{1}{\omega^{2}} \mathbf{u}
$$

On the other hand, if $\mathbf{u} \in \mathbf{L}^{2}(\Omega)$ satisfies (36), then $\mathbf{u} \in \mathbf{V}$ and $\mathbf{u}$ is an eigenfunction of $\mathbf{S}$. Due to the compactness of the embedding $\mathbf{V} \subset \mathbf{L}^{2}(\Omega)$, the operator $\mathbf{S}_{R}^{-1}$ is compact. Consequently, $\mathbf{S}_{R}^{-1}$ is a compact self-adjoint endomorphism on $\mathbf{L}^{2}(\Omega)$. Hence, in view of (35) the assertions can be deduced from the Hilbert-Schmidt theory and the Fredholm alternative (cf., e.g., [109]).

\subsection{Acoustic Streaming}

For the weak formulation of the periodic linearized compressible Navier-Stokes equations (16a)-(16e), we introduce the function spaces

$$
\begin{aligned}
\mathbf{V}_{\mathbf{g}_{1}}:= & \left\{\mathbf{v} \in H^{1}\left(\left(0, T_{1}\right) ; \mathbf{H}^{-1}(\Omega)\right) \cap\right. \\
& \left.L^{2}\left(\left(0, T_{1}\right) ; \mathbf{H}^{1}(\Omega)\right)|\mathbf{v}|_{\Gamma_{D}}=\mathbf{g}_{1}\right\}, \\
W:= & H^{1}\left(\left(0, T_{1}\right) ; L^{2}(\Omega)\right) .
\end{aligned}
$$

The weak formulation of (16a)-(16e) amounts to the computation of $\left(\mathbf{v}_{1}, p_{1}\right) \in \mathbf{V}_{\mathbf{g}_{1}} \times W$ such that for all $\mathbf{w} \in \mathbf{H}_{0, \Gamma_{D}}^{1}(\Omega)$ and $q \in L^{2}(\Omega)$

$$
\begin{array}{r}
\left\langle\rho_{0} \frac{\partial \mathbf{v}_{1}}{\partial t}, \mathbf{w}\right\rangle+a\left(\mathbf{v}_{1}, \mathbf{w}\right)+b\left(p_{1}, \mathbf{w}\right)=\mathbf{0} \\
\left(\rho_{0}^{-1} c_{0}^{-2} \frac{\partial p_{1}}{\partial t}, q\right)_{0, \Omega_{2}}-b\left(q, \mathbf{v}_{1}\right)=0 \\
\mathbf{v}_{1}(\cdot, 0)=\mathbf{0}, \quad p_{1}(\cdot, 0)=0 .
\end{array}
$$

Here, $\langle\cdot, \cdot\rangle$ stands for the respective dual pairing, and the bilinear forms $a(\cdot, \cdot)$ and $b(\cdot, \cdot)$ are given by

$$
\begin{aligned}
a(\mathbf{v}, \mathbf{w}):= & \eta \int_{\Omega_{2}} \nabla \mathbf{v}: \nabla \mathbf{w} d x \\
& +\left(\xi+\frac{\eta}{3}\right) \int_{\Omega_{2}} \nabla \cdot \mathbf{v} \nabla \cdot \mathbf{w} d x, \\
b(p, \mathbf{w}):= & -\int_{\Omega_{2}} p \nabla \cdot \mathbf{w} d x .
\end{aligned}
$$

Theorem 3.4 For the solution of the variational problem (37a)-(37c) there holds:

If $\mathbf{g}_{1} \in \mathbf{L}^{2}\left(\left(0, T_{1}\right) ; \mathbf{H}_{00}^{1 / 2}\left(\Gamma_{D}\right)\right)$, then there exists a unique solution $\left(\mathbf{v}_{1}, p_{1}\right) \in \mathbf{V}_{\mathbf{g}_{1}} \times W$ of (37a)-(37c) satisfying the stability estimate

$$
\left\|\left(\mathbf{v}_{1}, p_{1}\right)\right\| \mathbf{v}_{\mathbf{g}_{1}} \times W \leq C_{T_{1}}\left\|\mathbf{g}_{1}\right\|_{\mathbf{L}^{2}\left(\left(0, T_{1}\right) ; \mathbf{H}_{00}^{1 / 2}\left(\Gamma_{D}\right)\right)},
$$

where $C_{T_{1}}>0$ is a constant depending on $T_{1}$.

Proof: The existence can be shown by the Galerkin method, whereas the uniqueness and the stability estimate (39) can be derived using the ellipticity of the bilinear form $a(\cdot, \cdot)$ and the fact that the bilinear form $b(\cdot, \cdot)$ satisfies an inf-sup condition.

On the other hand, setting

$$
\begin{aligned}
\mathbf{V}_{\mathbf{g}_{2}}:= & \left\{\mathbf{v} \in H^{1}\left(\left(0, T_{2}\right) ; \mathbf{H}^{-1}(\Omega)\right) \cap\right. \\
& \left.L^{2}\left(\left(0, T_{2}\right) ; \mathbf{H}^{1}(\Omega)\right)|\mathbf{v}|_{\Gamma_{D}}=\mathbf{g}_{2}\right\}, \\
W:= & H^{1}\left(\left(0, T_{2}\right) ; L^{2}(\Omega)\right)
\end{aligned}
$$

the weak formulation of the compressible Stokes system (18a)-(18e) requires the computation of 
$\left(\mathbf{v}_{2}, p_{2}\right) \in \mathbf{V}_{\mathbf{g}_{2}} \times W$ such that for all $\mathbf{w} \in \mathbf{H}_{0, \Gamma_{D}}^{1}(\Omega)$ and $q \in L^{2}(\Omega)$

$$
\begin{aligned}
\left\langle\rho_{0} \frac{\partial \mathbf{v}_{2}}{\partial t}, \mathbf{w}\right\rangle+a\left(\mathbf{v}_{2}, \mathbf{w}\right) & \\
+b\left(p_{2}, \mathbf{w}\right) & =(\mathbf{f}, \mathbf{w})_{0, \Omega}, \\
b\left(q, \mathbf{v}_{2}\right) & =(f, q)_{0, \Omega}, \\
\mathbf{v}_{2}(\cdot, 0)=\mathbf{0}, \quad p_{2}(\cdot, 0) & =0 .
\end{aligned}
$$

Here, the bilinear forms $a(\cdot, \cdot), b(\cdot, \cdot)$ are as in (38a),(38b), and the right-hand sides $\mathbf{f}, f$ are given by

$$
\begin{aligned}
& \mathbf{f}:=-\left\langle\rho_{1} \frac{\partial \mathbf{v}_{1}}{\partial t}+\rho_{0}\left(\nabla \mathbf{v}_{1}\right) \mathbf{v}_{1}\right\rangle, \\
& f:=-\left\langle\rho_{0}^{-1} \nabla \cdot\left(\rho_{1} \mathbf{v}_{1}\right)\right\rangle .
\end{aligned}
$$

Theorem 3.5 If $\mathbf{f} \in \mathbf{L}^{2}(\Omega), f \in L^{2}(\Omega)$, and $\mathbf{g}_{2} \in$ $\mathbf{H}_{00}^{1 / 2}\left(\Gamma_{D}\right)$, the weak formulation (40a)-(40c) of the compressible Stokes system admits a unique solution $\left(\mathbf{v}_{2}, p_{2}\right) \in \mathbf{V}_{\mathbf{g}_{2}} \times W$. Moreover, there exists a constant $C_{T_{2}}>0$ depending on $T_{2}$ such that

$$
\begin{aligned}
& \left\|\left(\mathbf{v}_{2}, p_{2}\right)\right\| \mathbf{v}_{\mathbf{g}_{2} \times W} \leq \\
& C_{T_{2}}\left(\|\mathbf{f}\|_{0, \Omega}+\|f\|_{0, \Omega}+\left\|\mathbf{g}_{2}\right\|_{\mathbf{H}_{00}^{1 / 2}\left(\Gamma_{D}\right)}\right) .
\end{aligned}
$$

Proof: The proof follows along the same lines as that of Theorem 3.4.

\section{Numerics}

\subsection{Surface Acoustic Waves}

We consider the case where the computational domain $\Omega \subset \mathbb{R}^{d}$ is a polygonal resp. polyhedral domain. We assume $\mathscr{T}_{h}(\Omega)$ to be a simplicial triangulation of $\Omega$ that aligns with $\Gamma_{u}$ and $\Gamma_{\phi}$ and denote by $S_{0 . \Gamma^{\prime}}^{(k)}\left(\Omega ; \mathscr{T}_{h}(\Omega)\right), k \in \mathbb{N}, \Gamma^{\prime}=\Gamma_{u}$ or $\Gamma^{\prime}=\Gamma_{\Phi}$ the finite element space of continuous functions $v_{h}: \Omega \rightarrow \mathbb{C}$ vanishing on $\Gamma^{\prime}$ and satisfying $\left.v_{h}\right|_{T} \in P_{k}(T), T \in$ $\mathscr{T}_{h}(\Omega)$, where $P_{k}(T)$ stands for the linear space of complex valued polynomials of degree $\leq k$ on $T \in$ $\mathscr{T}_{h}(\Omega)$. We approximate the space $\mathbf{V}$ of displacements and the space $W$ of electric potentials by

$$
\begin{aligned}
\mathbf{V}_{h} & :=S_{0, \Gamma_{u}}^{(k)}\left(\Omega ; \mathscr{T}_{h}(\Omega)\right)^{d}, \\
W_{h} & :=S_{0, \Gamma_{\Phi}}^{(k)}\left(\Omega ; \mathscr{T}_{h}(\Omega)\right) .
\end{aligned}
$$

We refer to $\mathbf{A}_{h}: \mathbf{V}_{h} \rightarrow \mathbf{V}_{h}^{*}, \mathbf{B}_{h}: W_{h} \rightarrow \mathbf{V}_{h}^{*}$ and $\mathbf{C}_{h}: W_{h} \rightarrow W_{h}^{*}$ as the operators associated with the sesquilinear forms $\left.a\right|_{\mathbf{V}_{h} \times \mathbf{V}_{h}},\left.b\right|_{W_{h} \times \mathbf{V}_{h}}$ and $\left.c\right|_{W_{h} \times W_{h}}$. These operators inherit their properties from their continuous counterparts. In particular, $\mathbf{A}_{h}, \mathbf{B}_{h}$ and $\mathbf{C}_{h}$ are bounded linear operators. Moreover, $\mathbf{A}_{h}$ is symmetric and $\mathbf{V}_{h}$-elliptic, whereas $\mathbf{C}_{h}$ is symmetric and $W_{h}$-elliptic having the same ellipticity constants $\gamma_{A}$ and $\gamma_{C}$. We further define $\mathbf{f}_{h} \in \mathbf{V}_{h}^{*}$ and $g_{h} \in W_{h}^{*}$ by

$$
\begin{aligned}
<\mathbf{f}_{h}, \mathbf{u}_{h}>:=<\mathbf{f}, \mathbf{u}_{h}>, \quad \mathbf{u}_{h} \in \mathbf{V}_{h}, \\
<g_{h}, \Phi_{h}>:=<g, \Phi_{h}>, \quad \Phi_{h} \in W_{h} .
\end{aligned}
$$

The finite element approximation of (28) requires the computation of $\mathbf{u}_{h} \in \mathbf{V}_{h}$ and $\Phi_{h} \in W_{h}$ such that

$$
\begin{aligned}
\left(\mathbf{A}_{h}-\omega^{2} \mathbf{I}_{h}\right) \mathbf{u}_{h}+\mathbf{B}_{h} \Phi_{h} & =\mathbf{f}_{h}, \\
\mathbf{B}_{h}^{*} \mathbf{u}_{h}-\mathbf{C}_{h} \Phi_{h} & =g_{h},
\end{aligned}
$$

where $\mathbf{I}_{h}$ is the injection $\mathbf{I}_{h}: \mathbf{V}_{h} \rightarrow \mathbf{V}_{h}^{*}$.

Static condensation of $\Phi_{h}$ results in the discrete Schur complement system

$$
\left(\mathbf{S}_{h}-\omega_{s}^{2} \mathbf{I}_{h}\right) \mathbf{u}_{h}=\mathbf{F}_{h},
$$

where $\mathbf{F}_{h}:=\mathbf{f}_{h}+\mathbf{B}_{h} \mathbf{C}_{h}^{-1} g_{h}$, and the discrete Schur complement $\mathbf{S}_{h}$ is given by

$$
\mathbf{S}_{h}:=\mathbf{A}_{h}+\mathbf{B}_{h} \mathbf{C}_{h}^{-1} \mathbf{B}_{h}^{*} .
$$

Obviously, $\mathbf{S}_{h}$ as given by (45) is the Galerkin approximation of $\mathbf{S}$, i.e.,

$$
<\mathbf{S}_{h} \mathbf{v}_{h}, \mathbf{w}_{h}>=<\mathbf{S v}_{h}, \mathbf{w}_{h}>, \mathbf{v}_{h}, \mathbf{w}_{h} \in \mathbf{V}_{h} .
$$

If $\omega \in \mathbb{R}$ is not an eigenvalue, then it is well-known that the operator $\mathbf{S}_{\omega}:=\mathbf{S}-\omega^{2} \mathbf{I}$ satisfies the inf-sup condition (cf., e.g., [25])

$$
\inf _{\mathbf{0} \neq \mathbf{v} \in \mathbf{V}} \sup _{\mathbf{0} \neq \mathbf{w} \in \mathbf{V}} \frac{\left|<\mathbf{S} \mathbf{S}_{\omega} \mathbf{v}, \mathbf{w}>\right|}{\|\mathbf{v}\|_{1, \Omega}\|\mathbf{w}\|_{1, \Omega}} \geq \beta>0,
$$

and for sufficiently small $h$ a discrete inf-sup condition holds true as well [70].

Theorem 4.1 Assume that for some $\omega \in \mathbb{R}$ the operator $\mathbf{S}_{\omega}$ satisfies the inf-sup condition (46). Then, there exist $h_{0}>0$ and $\beta_{\min }>0$ such that for all $h \leq h_{0}$ the operator $\mathbf{S}_{h, \omega}:=\mathbf{S}_{h}-\omega^{2} \mathbf{I}_{h}$ satisfies

$$
\inf _{\mathbf{0} \neq \mathbf{v}_{h} \in \mathbf{V}_{h}} \sup _{\mathbf{0} \neq \mathbf{w}_{h} \in \mathbf{V}_{h}} \frac{\left|<\mathbf{S}_{h, \omega} \mathbf{v}_{h}, \mathbf{w}_{h}>\right|}{\left\|\mathbf{v}_{h}\right\|_{1, \Omega}\left\|\mathbf{w}_{h}\right\|_{1, \Omega}} \geq \beta_{h} \geq \beta_{\text {min }} .
$$

The discrete system (43a),(43b) represents an algebraic saddle point problem of the form

$$
\left(\begin{array}{cc}
\mathbf{A} & \mathbf{B} \\
\mathbf{B}^{T} & -\mathbf{C}
\end{array}\right)\left(\begin{array}{c}
\mathbf{u} \\
\Phi
\end{array}\right)=\left(\begin{array}{l}
\mathbf{f} \\
\mathbf{g}
\end{array}\right)
$$


or $\mathbf{Z U}=\ell$, where $\mathbf{A} \in \mathbb{R}^{n \times n}$ and $\mathbf{C} \in \mathbb{R}^{m \times m}$ are symmetric, positive definite matrices satisfying

$$
\begin{gathered}
\gamma_{1} \mathbf{v}^{T} \mathbf{v} \leq \mathbf{v}^{T} \mathbf{A} \mathbf{v} \leq \Gamma_{1} \mathbf{v}^{T} \mathbf{v}, \mathbf{v} \in \mathbb{R}^{n} \\
\gamma_{2} \Phi^{T} \Phi \leq \Phi^{T} \mathbf{C} \Phi \leq \Gamma_{2} \Phi^{T} \Phi, \Phi \in \mathbb{R}^{m}
\end{gathered}
$$

with constants $0<\gamma_{i} \leq \Gamma_{i}, 1 \leq i \leq 2$. Moreover, $\mathbf{B} \in$ $\mathbb{R}^{n \times m}$ and $\mathbf{f} \in \mathbb{R}^{n}, \mathbf{g} \in \mathbb{R}^{m}$ whence $\mathbf{Z} \in \mathbb{R}^{N \times N}, \ell \in$ $\mathbb{R}^{N}$ where $N:=n+m$. We further assume that $\mathbf{Z}$ satisfies

$$
\inf _{\mathbf{U} \neq \mathbf{0}} \sup _{\mathbf{V} \neq \mathbf{0}} \frac{\left|\mathbf{V}^{T} \mathbf{Z U}\right|}{\|\mathbf{U}\|\|\mathbf{V}\|} \geq \gamma_{Z}>0
$$

where $\|\cdot\|$ stands for the Euclidean norm in $\mathbb{R}^{N}$.

Generalized saddle point problems such as (47) arise in the framework of stabilized Stokes systems $[92,93]$ or in mixed finite element approximations of boundary value problems for elliptic equations and systems [25]. We refer to [20, 32, 83] for basic results and to $[14,23,30,38,70]$ for efficient iterative solution techniques including multilevel preconditioning.

In the sequel, following [70, 93] we consider blockdiagonal preconditioners of the form

$$
\mathbf{P}^{-1}:=\left(\begin{array}{cc}
\tilde{\mathbf{A}} & \mathbf{0} \\
\mathbf{0} & \tilde{\mathbf{C}}
\end{array}\right)
$$

where we assume that $\tilde{\mathbf{A}} \in \mathbb{R}^{n \times n}$ and $\tilde{\mathbf{C}} \in \mathbb{R}^{m \times m}$ are symmetric, positive definite matrices satisfying

$$
\begin{gathered}
\tilde{\gamma}_{1} \mathbf{v}^{T} \mathbf{v} \leq \mathbf{v}^{T} \tilde{\mathbf{A}} \mathbf{v} \leq \tilde{\Gamma}_{1} \mathbf{v}^{T} \mathbf{v}, \mathbf{v} \in \mathbb{R}^{n} \\
\tilde{\gamma}_{2} \Phi^{T} \Phi \leq \Phi^{T} \tilde{\mathbf{C}} \Phi \leq \tilde{\Gamma}_{2} \Phi^{T} \Phi, \Phi \in \mathbb{R}^{m}
\end{gathered}
$$

with constants $0<\tilde{\gamma}_{i} \leq \tilde{\Gamma}_{i}, 1 \leq i \leq 2$.

As a consequence from (51) we deduce that $\mathbf{P}^{-1}$ is positive definite with

$$
\Gamma_{P}^{-1} \mathbf{z}^{T} \mathbf{z} \leq \mathbf{z}^{T} \mathbf{P}^{-1} \mathbf{z} \leq \gamma_{P}^{-1} \mathbf{z}^{T} \mathbf{z}, \quad \mathbf{z} \in \mathbb{R}^{N},
$$

where $\gamma_{P}^{-1}:=\max \left(\tilde{\Gamma}_{1}, \tilde{\Gamma}_{2}\right)$ and

$$
\Gamma_{P}^{-1}:=1 /\left(\min \left(\tilde{\gamma}_{1}, \tilde{\gamma}_{2}\right)\right) .
$$

In view of (49) and (52), lower and upper bounds for the spectrum of the preconditioned matrix $\mathbf{P}^{1 / 2} \mathbf{Z} \mathbf{P}^{1 / 2}$ follow readily.

Theorem 4.2 Under the assumptions (49) and (52), for $\mathbf{V} \in \mathbb{R}^{N}$ there holds

$$
\begin{aligned}
& \qquad \gamma_{P Z} \mathbf{V}^{T} \mathbf{V} \leq \mathbf{V}^{T} \mathbf{P}^{1 / 2} \mathbf{Z} \mathbf{P}^{1 / 2} \mathbf{V} \leq \Gamma_{P Z} \mathbf{V}^{T} \mathbf{V}, \\
& \text { where } \gamma_{P Z}:=\gamma_{P} \gamma_{Z} \text { and } \Gamma_{P Z}:=\Gamma_{P}\|\mathbf{Z}\| .
\end{aligned}
$$

Proof: By straightforward computation

$$
\begin{aligned}
& \inf _{\mathbf{W} \neq \mathbf{0}} \sup _{\mathbf{V} \neq \mathbf{0}} \frac{\left|\mathbf{V}^{T} \mathbf{P}^{1 / 2} \mathbf{Z} \mathbf{P}^{1 / 2} \mathbf{W}\right|}{\|\mathbf{V}\|\|\mathbf{W}\|} \\
& =\inf _{\tilde{\mathbf{W}} \neq \mathbf{0}} \sup _{\mathbf{\mathbf { V }} \neq \mathbf{0}} \frac{\left|\tilde{\mathbf{V}}^{T} \mathbf{Z} \tilde{\mathbf{W}}\right|}{\left(\tilde{\mathbf{V}}^{T} \mathbf{P}^{-1} \tilde{\mathbf{V}}\right)^{1 / 2}\left(\tilde{\mathbf{W}}^{T} \mathbf{P}^{-1} \tilde{\mathbf{W}}\right)^{1 / 2}} \\
& \geq \gamma_{P} \inf _{\tilde{\mathbf{W}} \neq \mathbf{0} \tilde{\mathbf{V}} \neq \mathbf{0}} \frac{\left|\tilde{\mathbf{V}} \tilde{\mathbf{V}}^{T} \mathbf{Z} \tilde{\mathbf{W}}\right|}{\|\tilde{\mathbf{V}}\|\|\tilde{\mathbf{W}}\|} \geq \gamma_{P} \gamma_{Z} .
\end{aligned}
$$

Similar arguments result in the upper bound in (53).

We allow the following inf-sup condition for the sesquilinear form $b(\cdot, \cdot)$ restricted to $\mathbf{V}_{h} \times W_{h}$ :

$$
\inf _{\mathbf{v}_{h} \in \mathbf{V}_{h}} \sup _{\varphi_{h} \in W_{h}} \frac{\left|b\left(\mathbf{v}_{h}, \varphi_{h}\right)\right|}{\left\|\mathbf{v}_{h}\right\| \mathbf{v}\left\|\varphi_{h}\right\|_{W}} \geq \beta_{h} \geq \beta_{\min } \geq 0,
$$

i.e., $\beta_{\min }=0$ is admitted. In this case, the associated matrix $\mathbf{B}$ may have a non-trivial kernel.

Lemma 4.3 Under the assumptions (48b) and (54), for $\mathbf{v} \in \mathbb{R}^{n}, \mathbf{v} \neq \mathbf{0}$ there holds

$$
\frac{\beta_{\min }^{2}}{\|\mathbf{C}\|} \leq \frac{\mathbf{v}^{T} \mathbf{B C}^{-1} \mathbf{B}^{T} \mathbf{v}}{\mathbf{v}^{T} \mathbf{v}} \leq \frac{\|\mathbf{B}\|^{2}}{\gamma_{2}} .
$$

Proof: The assertion follows from

$$
\begin{aligned}
& \mathbf{v}^{T} \mathbf{B} \mathbf{C}^{-1} \mathbf{B}^{T} \mathbf{v}=\sup _{\Phi \neq \mathbf{0}} \frac{\left(\Phi^{T} \mathbf{C}^{1 / 2} \mathbf{C}^{-1 / 2} \mathbf{B}^{T} \mathbf{v}\right)^{2}}{\Phi^{T} \mathbf{C} \Phi}, \\
& \geq \frac{1}{\|\mathbf{C}\|} \sup _{\Phi \neq \mathbf{0}} \frac{\left(\Phi^{T} \mathbf{B}^{T} \mathbf{v}\right)^{2}}{\Phi^{T} \Phi} \geq \frac{\beta_{\min }^{2}}{\|\mathbf{C}\|} \mathbf{v}^{T} \mathbf{v},
\end{aligned}
$$

and

$$
\mathbf{v}^{T} \mathbf{B} \mathbf{C}^{-1} \mathbf{B}^{T} \mathbf{v} \leq \frac{1}{\gamma_{2}}\left\|\mathbf{B}^{T} \mathbf{v}\right\|^{2} \leq \frac{\|\mathbf{B}\|^{2}}{\gamma_{2}} \mathbf{v}^{T} \mathbf{v} .
$$

The preconditioned saddle point system is given by

$$
\begin{aligned}
& \left(\begin{array}{cc}
\tilde{\mathbf{A}}^{-1} \mathbf{A} & \tilde{\mathbf{A}}^{-1} \mathbf{B} \\
\tilde{\mathbf{C}}^{-1} \mathbf{B}^{T} & -\tilde{\mathbf{C}}^{-1} \mathbf{C}
\end{array}\right)\left(\begin{array}{c}
\mathbf{u} \\
\Phi
\end{array}\right)= \\
& \left(\begin{array}{c}
\tilde{\mathbf{A}}^{-1} \mathbf{f} \\
\tilde{\mathbf{C}}^{-1} \mathbf{g}
\end{array}\right) .
\end{aligned}
$$

The Schur complement matrix of the preconditioned system reads $\tilde{\mathbf{S}}=\tilde{\mathbf{A}}^{-1} \mathbf{S}$. The spectrum of $\tilde{\mathbf{A}}^{-1} \mathbf{S}$ can be determined from the eigenvalues of $\tilde{\mathbf{A}}^{-1 / 2} \mathbf{S} \tilde{\mathbf{A}}^{-1 / 2}$. 
Theorem 4.4 The following lower and upper bounds apply

$$
\gamma_{P S} \leq \frac{\mathbf{v}^{T} \tilde{\mathbf{S}} \mathbf{v}}{\|\mathbf{v}\|^{2}} \leq \Gamma_{P S}
$$

where

$$
\begin{aligned}
\gamma_{P S} & :=\frac{1}{\|\tilde{\mathbf{A}}\|}\left(\gamma_{1}+\frac{\beta_{\min }^{2}}{\|\mathbf{C}\|}\right), \\
\Gamma_{P S} & :=\frac{1}{\tilde{\gamma}_{1}}\left(\|\mathbf{A}\|+\frac{\|\mathbf{B}\|^{2}}{\gamma_{2}}\right) .
\end{aligned}
$$

Proof: Setting $\mathbf{w}:=\tilde{\mathbf{A}}^{-1 / 2} \mathbf{v}$, we have

$$
\begin{aligned}
& \frac{\mathbf{v}^{T} \tilde{\mathbf{A}}^{-1 / 2} \mathbf{S} \tilde{\mathbf{A}}^{-1 / 2} \mathbf{v}}{\mathbf{v}^{T} \mathbf{v}} \\
= & \frac{\mathbf{v}^{T} \tilde{\mathbf{A}}^{-1 / 2} \mathbf{A} \tilde{\mathbf{A}}^{-1 / 2} \mathbf{v}}{\mathbf{v}^{T} \mathbf{v}} \\
& +\frac{\mathbf{v}^{T} \tilde{\mathbf{A}}^{-1 / 2} \mathbf{B} \mathbf{C}^{-1} \mathbf{B}^{T} \tilde{\mathbf{A}}^{-1 / 2} \mathbf{v}}{\mathbf{v}^{T} \mathbf{v}} \\
= & \frac{\mathbf{w}^{T} \mathbf{A} \mathbf{w}}{\mathbf{w}^{T} \tilde{\mathbf{A}} \mathbf{w}}+\frac{\mathbf{w}^{T} \mathbf{B} \mathbf{C}^{-1} \mathbf{B}^{T} \mathbf{w}}{\mathbf{w}^{T} \tilde{\mathbf{A}} \mathbf{w}} .
\end{aligned}
$$

The first term can be estimated by the ellipticity properties of $\mathbf{A}$ and $\tilde{\mathbf{A}}$,

$$
\frac{\gamma_{1}}{\|\tilde{\mathbf{A}}\|} \leq \frac{\mathbf{w}^{T} \mathbf{A} \mathbf{w}}{\mathbf{w}^{T} \tilde{\mathbf{A}} \mathbf{w}} \leq \frac{\|\mathbf{A}\|}{\tilde{\gamma}_{1}} .
$$

In view of Lemma 4.3 and the ellipticity of $\tilde{\mathbf{A}}$, for the second term we obtain

$$
\frac{\beta_{\min }^{2}}{\|\tilde{\mathbf{A}}\|\|\mathbf{C}\|} \leq \frac{\mathbf{w}^{T} \mathbf{B} \mathbf{C}^{-1} \mathbf{B}^{T} \mathbf{w}}{\mathbf{w}^{T} \tilde{\mathbf{A}} \mathbf{w}} \leq \frac{\|\mathbf{B}\|^{2}}{\tilde{\gamma}_{1} \gamma_{2}} .
$$

The assertion follows from the above estimates.

Particular interest is in such preconditioners where the lower and upper bounds $\gamma_{P Z}$ and $\Gamma_{P Z}$ for the spectrum of the preconditioned saddle point matrix PZ and the corresponding bounds $\gamma_{P S}$ and $\Gamma_{P S}$ for the spectrum of the preconditioned Schur complement $\tilde{\mathbf{A}}^{-1} \mathbf{S}$ as well as the bounds for the spectrum of $\tilde{\mathbf{C}}^{-1} \mathbf{C}$ are independent of the mesh size $h$. Such preconditioners are provided by multilevel preconditioners of PBX-type with respect to a nested hierarchy of simplicial triangulations of the computational domain $\Omega$ (cf., e.g., [24, 85]).

For the surface acoustic wave device we have used a reduced model in the $\left(x_{1}, x_{3}\right)$-plane assuming that all variables do not depend on $x_{2}$ and have no impact in the $x_{2}$-direction. The piezoelectric material is lithium niobate $\left(\mathrm{LiNbO}_{3}\right)$ with density $\rho=4630 \frac{\mathrm{kg}}{\mathrm{m}^{3}}$.
The used crystal cut is $\mathbf{Y X} l 128^{\circ} \mathrm{LiNbO}_{3}$. The length $\ell$ and the height $h$ of the SAW chip have been chosen according to $\ell=1.2 \mathrm{~mm}$ and $h=0.6 \mathrm{~mm}$ so that $\Omega=(0,1.2) \times(0,0.6)$. The Dirichlet and Neumann boundary conditions have been specified according to

$$
\begin{gathered}
\mathbf{u}_{\Gamma}(\mathbf{x}, t)=\mathbf{0} \quad \text { on } \Gamma_{u} \quad, \quad \sigma_{\mathbf{n}}(\mathbf{x}, t)=\mathbf{0} \quad \text { on } \Gamma_{\sigma}, \\
\Phi_{\Gamma}(\mathbf{x}, t)=\hat{\Phi} \sin \left(\frac{2 \pi}{\lambda_{I D T}}\right) \sin (\omega t) \quad \text { on } \Gamma_{\Phi}^{(1)}, \\
\Phi_{\Gamma}(\mathbf{x}, t)=0 \quad \text { on } \Gamma_{\Phi}^{(2)}, \quad D_{\mathbf{n}}(\mathbf{x}, t)=0 \quad \text { on } \Gamma_{D},
\end{gathered}
$$

where the Dirichlet boundaries are $\Gamma_{u}:=[0,1.2] \times$ $\{0\}, \Gamma_{\Phi}^{(1)}:=[0.2,0.4] \times\{1.2\}, \Gamma_{\Phi}^{(2)}:=[0,1.2] \times$ $\{0\}$, whereas $\Gamma_{\sigma}:=\partial \Omega \backslash \Gamma_{u}$ and $\Gamma_{D}=\partial \Omega \backslash\left(\Gamma_{\Phi}^{(1)} \cup\right.$ $\left.\Gamma_{\Phi}^{(2)}\right)$ stand for the Neumann boundaries.

Concerning the wavelength $\lambda_{I D T}$ of the interdigital transducer and its operating frequency $f$, we have made the realistic choice $\lambda_{I D T}=40 \mu \mathrm{m}$ and $f=$ $\frac{\omega}{2 \pi}=100 \mathrm{MHz}$.

The computational domain has been discretized by a nested hierarchy of simplicial triangulations of the computational domain $\Omega$ generated by uniform refinement of a given coarse mesh. It is well-known that for time-harmonic waves with increasing angular frequency $\omega=\frac{2 \pi f}{\lambda}$ the finite element error grows, even if we account for a condition on the meshsize like $h \lesssim \lambda$. A common choice is $h \lesssim \frac{\lambda}{2}$. However, an intrinsic analysis shows that an additional condition like $h \lesssim \sqrt{\lambda^{3}}$ is needed, if we want to control the finite element error (cf. [68]). Therefore, we have chosen the meshsize for the coarsest grid of the nested hierarchy accordingly.

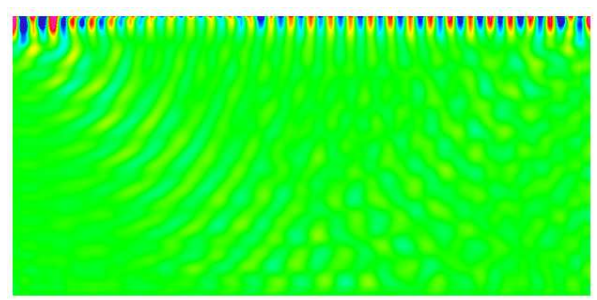

Fig. (9): Electric potential wave

Fig. (9)-(11) display the amplitudes of the electric potential and the polarized Rayleigh waves. The amplitudes of the displacement waves are in the region of nanometers. The SAWs are strictly confined to the surface of the substrate with a penetration depth in the range of one wavelength.

One of the most outstanding properties of surface 


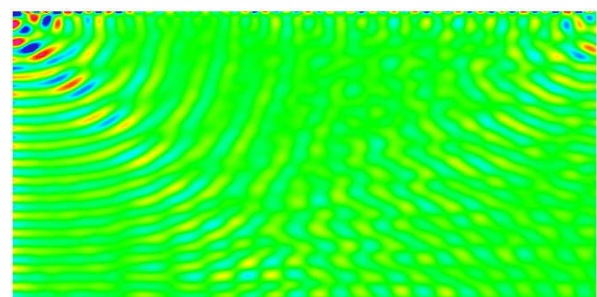

Fig. (10): Displacement wave amplitudes in $x_{1}$ direction

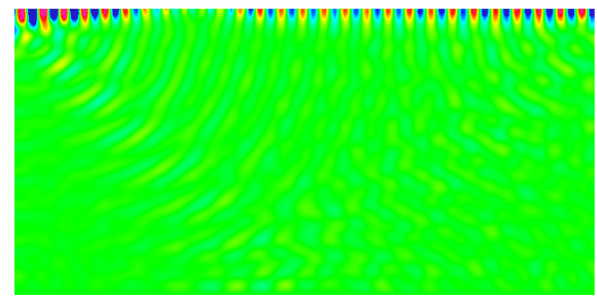

Fig. (11): Displacement wave amplitudes in $x_{2}$ direction

acoustic wave propagation on piezoelectric materials is that the velocity of the SAW is independent of the applied frequency. In case of $\mathbf{Y X} l 128^{\circ} \mathrm{LiNbO}_{3}$, the SAW velocity is given by $v=3992 \frac{\mathrm{m}}{\mathrm{s}}$, cf. [29]. Thus, for an excitation at frequency $f=100 \mathrm{MHz}$ the theoretical wavelength of the SAW is given by $\lambda=\frac{v}{f} \approx 40 \mu \mathrm{m}$. The computations show the same wavelength for the SAW. Fig. (12) also illustrates the piezoelectric wave for $f=50 \mathrm{MHz}$. The wavelength of the SAW for $f=100 \mathrm{MHz}$ is half of that for $f=50 \mathrm{MHz}$.

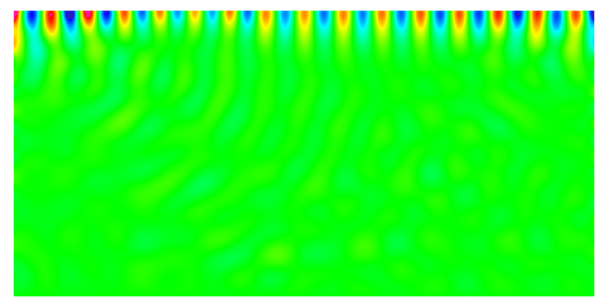

Fig. (12): Electric potential wave for $f=50 \mathrm{MHz}$

Remark 4.5 The wavelength of an occurring electro-magnetic wave is in the region of approximately $0.3 \mathrm{~m}$. Hence, the negligence of this electromagnetic wave in the modeling of piezoelectric SAW devices is justified.
Rayleigh surface waves characteristically show an elliptical displacement, i.e., the displacements in the $x_{1}$ - and $x_{2}$-direction are $90^{\circ}$ out of phase with one another. Additionally, the amplitude of the surface displacement in the $x_{2}$-direction is larger than that along the SAW propagation axis $x_{1}$. These observations are confirmed by the numerical computations as can be seen in Fig. (13) and (14). In Fig. (13), the displacements in the $x_{1}$ - and $x_{2}$-direction for a certain surface area are depicted. The $x_{2}$-displacements are flipped vertically for easier comparability. In

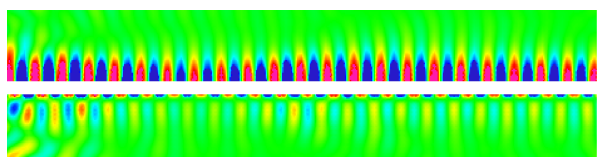

Fig. (13): Phaseshift of $x_{1}$ - and $x_{2}$ - (flipped) displacements

Fig. (14) a certain surface area is magnified and the vectors indicate the surface displacements.

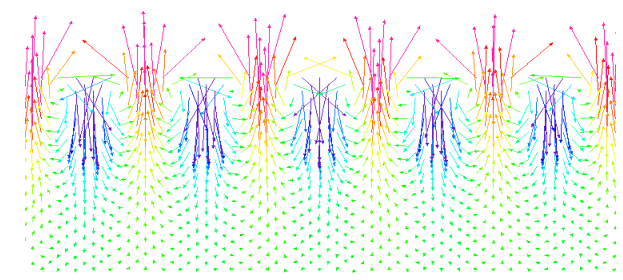

Fig. (14): Displacement vectors for the SAW

The excitation of an IDT on the surface of a piezoelectric material leads to the generation of bulk acoustic waves (BAWs) as well as surface acoustic waves. These bulk waves can also be observed in our simulations in Fig. (9)-(12).

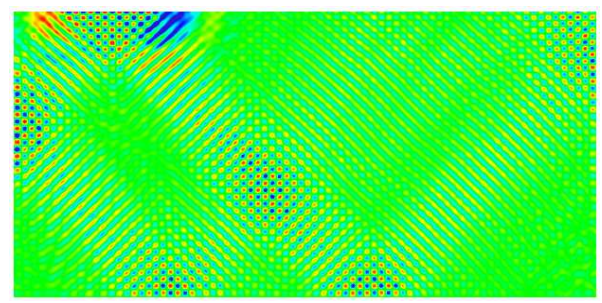

Fig. (15): Bulkwave Excitation

Technologically, they are desirably employed in solid-state circuits [29]. We refer to [39, 41, 57, 61, 
$62,76,99]$ for finite element approximations of surface acoustic wave propagation in signal processing. For the SAW devices under consideration, however, the occurrence of BAWs is unwanted, since the interference of BAWs with SAWs can lead to a complete loss of functionality of the device. The approach used here is sufficiently general to simulate every kind of piezoelectric resonator. In Fig. (15) we have used an $\mathbf{Y X} l 38^{\circ}$ cut of $\mathrm{LiNbO}_{3}$ to generate a strong bulk acoustic wave at a frequency $f=200$ MHz.

All numerical computations show relatively strong reflections from the boundaries of the SAW device. In real devices these reflections are usually avoided by attaching some adhesive material to the side boundaries. An easy way to model such a damping is to introduce an additional term (the socalled gyroscopic term) into the piezoelectric equations which now become

$$
\begin{aligned}
& \rho \frac{\partial^{2} u_{i}}{\partial t^{2}}-\frac{\partial \beta_{i}}{\partial x_{j}} \frac{\partial^{3} u_{i}}{\partial^{2} x_{j} \partial t}- \\
&-c_{i j k l} \frac{\partial^{2} u_{k}}{\partial x_{l} \partial x_{j}}-e_{k i j} \frac{\partial^{2} \Phi}{\partial x_{k} \partial x_{j}}=b_{i} \\
& e_{i k l} \frac{\partial^{2} u_{k}}{\partial x_{l} \partial x_{i}}-\varepsilon_{i j} \frac{\partial^{2} \Phi}{\partial x_{j} \partial x_{i}}=\beta_{i}
\end{aligned}
$$

cf. e.g. [15, 69]. Introducing such a damping at the boundaries of the bottom and left-hand side we indeed get less reflections and thus less disturbances for the SAW (compare the $x_{1}$-displacements in Fig. (10) (computations without damping) with the new computations with a damping term in Fig. (16)).

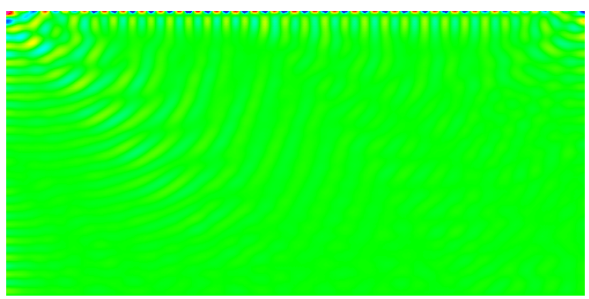

Fig. (16): $x_{1}$-displacements with damping

\subsection{Acoustic Streaming}

For the simulation of the SAW induced fluid flow in the microchannels of the biochip, we have numerically solved the two systems (16a)-(16e) and (18a)-(18e) obtained from the compressible NavierStokes equations (16a)-(16d) by the homogenization approach as described in subsection 2.2.
For discretization in space, we have used TaylorHood P2-P1 elements with respect to a geometrically conforming simplicial triangulation $\mathscr{T}_{h}\left(\Omega_{2}\right)$ of the computational domain $\Omega_{2}$ occupied by the fluid, i.e., we have chosen the finite element spaces

$\mathbf{V}_{h}:=\left\{\mathbf{v}_{h} \in C\left(\Omega_{2}\right)^{3}\left|\mathbf{v}_{h}\right|_{T} \in P_{2}(T)^{3}, T \in \mathscr{T}_{h}\left(\Omega_{2}\right)\right\}$, $W_{h}:=\left\{w_{h} \in C\left(\Omega_{2}\right)\left|w_{h}\right|_{T} \in P_{1}(T), T \in \mathscr{T}_{h}\left(\Omega_{2}\right)\right\}$,

and $\mathbf{V}_{\mathbf{g}_{h, i}}:=\mathbf{V}_{h} \cap \mathbf{V}_{\mathbf{g}_{h, i}}$ with $\mathbf{g}_{h, i}$ being a piecewise quadratic approximation of $\mathbf{g}_{i}, 1 \leq i \leq 2$ (cf., e.g., $[50,55])$. The boundary data have been chosen according to the computation of the displacements $\mathbf{u}_{h}$ obtained by the numerical solution of the piezoelectric equations as described in subsection 3.1. For discretization in time of (16a)-(16e), we have used the $\Theta$-scheme with respect to a uniform partitioning of the time interval of step size $k$ and a preferred choice of $\Theta=1$ (backward Euler scheme) or $\Theta=1 / 2$ (Crank-Nicolson). The chosen discretizations amount to the numerical solution of algebraic saddle point problems of the form

$$
\left(\begin{array}{cc}
\mathbf{A} & \mathbf{B} \\
\mathbf{B}^{T} & 0
\end{array}\right)\left(\begin{array}{l}
\mathbf{v} \\
\mathbf{p}
\end{array}\right)=\left(\begin{array}{l}
\mathbf{b}_{1} \\
\mathbf{b}_{2}
\end{array}\right)
$$

which has been done using a multilevel preconditioned inexact Uzawa algorithm [37] with respect to a hierarchy $\left\{\mathscr{T}_{h}\right\}_{i=0}^{\ell}$ of triangulations. The fluid in the microchannels has been assumed to be water with the relevant constants $\rho_{f}, \eta$, and $\xi$ as well as the sound velocity $c_{0}$ listed in Table 2 (cf. section 2).

For the simulations we have chosen $\Omega_{2}:=(0, L) \times$ $(0, W) \times(0, H)$ and $\Gamma_{2, D}:=\left[0, L_{1}\right] \times[0, W] \times\{0\}$ assuming a maximal displacement of the lower wall of $\varepsilon=1.0 \cdot 10^{-9} \mathrm{~m}$.
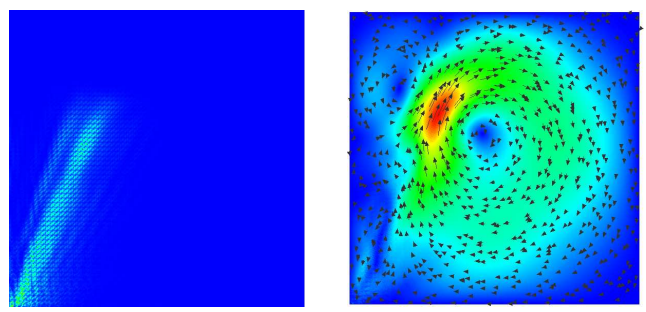

Fig. (17): Effective force (left) and associated velocity field (right)

For $L=W=H=2.5 \cdot 10^{2} \mu m$ and $L_{1}=0.25$. $10^{2} \mu \mathrm{m}$, Fig. (17) (left) shows the effective force which is the time-averaged sound velocity in the fluid, whereas Fig. (17) (right) displays the associated velocity field, both at the longitudinal section $[0, L] \times\{1.25\} \times[0, H]$. 
Fig. (18) illustrates the computed propagation of the SAWs in a microchannel where the SAWs penetrate the channel at its lower left end. The SAWs propagate from the left to the right and undergo a significant damping.

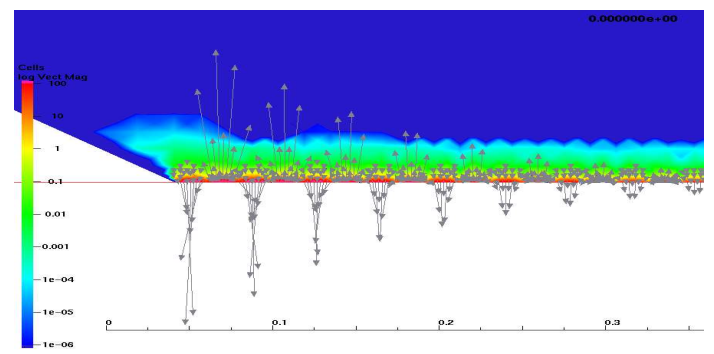

Fig. (18): Propagation and damping of SAWs in a microchannel

For model validation and verification of the computational results, we have compared the results of the numerical computations based on the numerical solution of our model equations with experimentally available data. The experimental setup consists of a part of a biochip with an IDT placed below its right lower corner (cf. Fig. (19) (left)). The SAW induced velocity field is visualized by tracer particles using a light microscope. Fig. (19) (right) displays the computed velocity field based on numerical computations for the discretized system (16a)-(16e) using the physical data underlying the experimental setup.
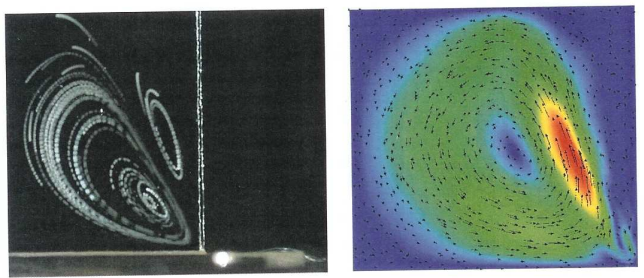

Fig. (19): Experimentally observed SAW in a microfluidic channel (left) and visualization of numerical computations (right).

\section{Optimization}

\subsection{Path-Following Barrier Methods}

Optimal design problems associated with fluid flow problems play an important role in a wide variety of engineering applications (cf., e.g., [81] and the references therein). A typical example is to design the geometry of the container of the fluid, e.g., a channel, a reservoir, or a network of channels and reservoirs, in such a way that a desired flow velocity and/or pressure profile is achieved. The solution of the problem amounts to the minimization of an objective functional that depends on the state variables (velocity, pressure) and on the design variables which determine the geometry. The state variables are supposed to satisfy the underlying fluid mechanical equations, and there are typically further constraints, e.g., bilateral constraints on the design variables which restrict the shape of the fluid filled domain.

Shape optimization problems have been extensively studied and are well documented in the literature (cf., e.g., the monographs $[1,16,31,33,58,59$, $81,87,94]$ ). The traditional approach relies on a separate treatment of the design objective and the state equation by an iterative cycle that starts from a given design, computes an approximate solution of the state equation for that design, invokes some sensitivity analysis for an update of the design, and continues this way until convergence is achieved. In contrast to this successive approximation, 'all-atonce methods' or 'one-shot methods' have attracted considerable attention in PDE constrained optimization whose characteristic feature is that the numerical solution of the state equation is an integral part of the optimization routine. In particular, it has been shown that this novel approach may lead to significant savings of computational time (see, e.g., $[21,22,64,65,66,91])$.

With the acoustic streaming subproblem (20a)-(20d) in mind, we focus on the optimal design of stationary fluid flow problems where for simplicity we consider the classical (incompressible) Stokes equations. The objective is to design the geometry of a channel or a particular geometric feature of a channel such that a desired profile of the velocity and/or the pressure can be achieved as closely as possible. The design variables are chosen as the Bézier control points of a globally continuous Bézier curve representation of the walls of the channel subject to bilateral constraints. We follow an 'all-at-once method' based on a barrier method where the Stokes system is coupled by Lagrange multipliers and the constraints on the design variables are taken care of by parameterized logarithmic barrier functions. This gives rise to a family of minimization subproblems parameterized by the barrier parameter. The optimality conditions represent a parameter dependent nonlinear system whose solution is the socalled barrier path (cf., e.g., [44, 106]). The numerical challenge is to follow the barrier path as the 
barrier parameter goes to zero. We use an adaptive continuation method with tangent continuation as a predictor and Newton's method as a corrector adopting ideas from [34].

In particular, we consider Stokes flow in a bounded domain $\Omega(\alpha) \subset \mathbb{R}^{2}$ with boundary

$$
\Gamma(\alpha)=\Gamma_{\text {in }}(\alpha) \cup \Gamma_{\text {lat }}(\alpha) \cup \Gamma_{\text {out }}(\alpha),
$$

depending on the design variable $\alpha=$ $\left(\alpha_{1}, \cdots, \alpha_{m}\right)^{T} \in \mathbb{R}^{m}$ which are chosen as the Bézier control points of a Bézier curve representation of the lateral boundaries of the domain. Denoting the viscosity of the fluid by $v$, the velocity by $\mathbf{u}$ and the pressure by $p$, we refer to

$$
\begin{aligned}
& J(v, p, \alpha):= \\
& \frac{\kappa_{1}}{2} \int_{\Omega(\alpha)}\left|v-v^{d}\right|^{2} d x+\frac{\kappa_{2}}{2} \int_{\Omega(\alpha)}\left|p-p^{d}\right|^{2} d x
\end{aligned}
$$

as the objective functional. Here, $v^{d}, p^{d}$ are desired velocity and pressure profiles, and $\kappa_{i}, 1 \leq i \leq 2$, stand for appropriately chosen weight factors. The shape optimization problem reads

$$
\text { minimize } J(v, p, \alpha)
$$

subject to the Stokes system (state equations)

$$
\begin{aligned}
-\nabla \cdot \sigma(v) & =0 \quad \text { in } \Omega(\alpha), \\
\nabla \cdot v & =0 \quad \text { in } \Omega(\alpha), \\
\sigma(v) & =-p \mathbf{I}+v \mathbf{D}(v), \\
\sigma(v) \cdot \mathbf{n} & =\mathbf{0} \quad \text { on } \Gamma_{\text {out }}(\alpha), \\
\mathbf{n} \cdot v & =\left\{\begin{array}{rl}
u_{\text {in }} & \text { on } \Gamma_{\text {in }}(\alpha) \\
0 & \text { on } \Gamma_{\text {lat }}(\alpha)
\end{array},\right. \\
\mathbf{t} \cdot v & =0 \quad \text { on } \Gamma(\alpha) .
\end{aligned}
$$

Here, $\Gamma_{\text {in }}(\alpha), \Gamma_{\text {out }}(\alpha), \Gamma_{\text {lat }}(\alpha)$ are the inflow, outflow, and lateral boundaries with $\mathbf{n}, \mathbf{t}$ denoting the exterior unit normal vector and the tangential unit vector, respectively. Moreover, we impose the bilateral constraints

$$
\alpha_{i}^{\min } \leq \alpha_{i} \leq \alpha_{i}^{\max }, 1 \leq i \leq m .
$$

It is well-known that the the weak formulation of (60a)-(60d) admits a unique solution (cf., e.g., [78]).

For the finite element approximation of (59)-(61) we choose $\hat{\alpha} \in K$ as a reference design and refer to $\hat{\Omega}:=\Omega(\hat{\alpha})$ as the associated reference domain. Then, the actual domain $\Omega(\alpha)$ can be obtained from the reference domain $\hat{\Omega}$ by means of a mapping
$\Omega(\alpha)=\Phi(\hat{\Omega} ; \alpha)$. The advantage of using the reference domain $\hat{\Omega}$ is that finite element approximations can be performed with respect to that fixed domain without being forced to remesh for every new set of the design variables.

For the discretization of the velocity $v$ and the pressure $p$, we use Taylor-Hood P2/P1 elements with respect to a shape regular family of simplicial triangulations of $\hat{\Omega}$.

We denote by $\mathbf{v} \in \mathbb{R}^{n_{1}}$ and $\mathbf{p} \in \mathbb{R}^{n_{2}}$ the vectors standing for the velocity components and the pressure in the nodal points associated with the Taylor-Hood finite element approximation of the Stokes system, and we refer to $\mathbf{J}(\mathbf{v}, \mathbf{p}, \alpha)$ as the discretized objective functional. Then, the discrete optimal design problem can be stated as follows:

$$
\operatorname{minimize} \mathbf{J}(\mathbf{v}, \mathbf{p}, \alpha)
$$

subject to the algebraic system (discretized Stokes equations)

$$
\begin{aligned}
& \mathbf{S}(\alpha) \mathbf{y}:= \\
& \left(\begin{array}{cc}
\mathbf{A}(\alpha) & \mathbf{B}^{T}(\alpha) \\
\mathbf{B}(\alpha) & 0
\end{array}\right)\left(\begin{array}{l}
\mathbf{v} \\
\mathbf{p}
\end{array}\right)=\mathbf{g},
\end{aligned}
$$

and subject to the inequality constraints (61).

Due to the nonlinear dependence on the design variables, (62), (63) and (61) represents an inequality constrained nonlinear programming problem. For its numerical solution we use a path-following barrier method as described below.

We couple the inequality constraints (61) by logarithmic barrier functions with a barrier parameter $\beta=1 / \mu>0, \mu \rightarrow \infty$, and the PDE constraint (60) by a Lagrange multiplier $\lambda=\left(\lambda_{\mathbf{v}}, \lambda_{\mathbf{p}}\right)^{T}$. This leads to the saddle point problem

$$
\inf _{\mathbf{y}, \alpha} \sup _{\lambda} L^{(\mu)}(\mathbf{y}, \lambda, \alpha)
$$

where $L^{(\mu)}$ stands for the Lagrangian

$$
L^{(\mu)}(\mathbf{y}, \lambda, \alpha)=B^{(\mu)}(\mathbf{y}, \alpha)+\langle S(\mathbf{y}, \alpha)-\mathbf{g}, \lambda\rangle,
$$

and $B^{(\mu)}(\mathbf{y}, \alpha)$ is the so-called barrier function as given by

$$
\begin{aligned}
& B^{(\mu)}(\mathbf{y}, \alpha):= \\
& J(\mathbf{y}, \alpha)-\frac{1}{\mu} \sum_{i=1}^{m}\left[\ln \left(\alpha_{i}-\alpha_{i}^{\min }\right)+\ln \left(\alpha_{i}^{\max }-\alpha_{i}\right)\right]
\end{aligned}
$$

(for details cf., e.g., [106]). The barrier path

$$
\mu \longmapsto \mathbf{x}(\mu):=(\mathbf{y}(\mu), \lambda(\mu), \alpha(\mu))^{T}
$$


is given as the solution of the nonlinear system

$$
F(\mathbf{x}(\mu), \mu)=\left(\begin{array}{c}
L_{\mathbf{y}}^{(\mu)}(\mathbf{y}, \lambda, \alpha) \\
L_{\lambda}^{(\mu)}(\mathbf{y}, \lambda, \alpha) \\
L_{\alpha}^{(\mu)}(\mathbf{y}, \lambda, \alpha)
\end{array}\right)=0
$$

Here, the subindices refer to the derivatives of the Lagrangian with respect to the primal, the dual, and the design variables. The choice of the barrier parameter strongly influences the performance of the method. There are static strategies with the FiaccoMcCormick approach as the most prominent one (cf. [43]), where the barrier parameter is fixed until an approximate solution of (64) has been obtained, and there is a variety of dynamic update strategies (cf. [12, 36, 48, 84, 96, 97, 98]). Convergence properties of the Fiacco-McCormick approach have been studied in [28] and [100], whereas a convergence analysis of dynamic update strategies has been addressed in [12, 36, 84, 97].

We consider the solution of (66) by an adaptive continuation method based on the affine invariant convergence theory of Newton-type methods.

The adaptive continuation method is a predictorcorrector method featuring an adaptively determined continuation step size in the predictor and Newton's method as a corrector. It relies on the affine invariant convergence theory of Newton and Newton-type methods (cf., e.g., [34]) and ensures that the iterates stay within a neighborhood of the barrier path so that convergence to a local minimum of the original minimization problem can be achieved (cf. Fig. (20)).

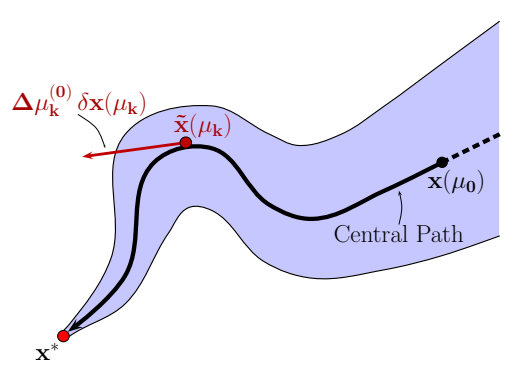

Fig. (20): Predictor step of the adaptive continuation method.

Predictor Step: The predictor step relies on tangent continuation along the trajectory of the Davidenko equation

$$
F_{\mathbf{x}}(\mathbf{x}(\mu), \mu) \mathbf{x}^{\prime}(\mu)=-F_{\mu}(\mathbf{x}(\mu), \mu) .
$$

It amounts to the implementation of an explicit Euler step: Given some approximation $\tilde{\mathbf{x}}\left(\mu_{k}\right)$ at $\mu_{k}>0$, compute $\tilde{\mathbf{x}}^{\left(j_{0}\right)}\left(\mu_{k+1}\right)$, where $\mu_{k+1}=\mu_{k}+\Delta \mu_{k}^{(j)}$, according to

$$
\begin{aligned}
& F_{\mathbf{x}}\left(\tilde{\mathbf{x}}\left(\mu_{k}\right), \mu_{k}\right) \delta \mathbf{x}\left(\mu_{k}\right)=-F_{\mu}\left(\tilde{\mathbf{x}}\left(\mu_{k}\right), \mu_{k}\right), \\
& \tilde{\mathbf{x}}^{\left(j_{0}\right)}\left(\mu_{k+1}\right)=\tilde{\mathbf{x}}\left(\mu_{k}\right)+\Delta \mu_{k}^{(j)} \delta \mathbf{x}\left(\mu_{k}\right),
\end{aligned}
$$

starting with $j=0(j \geq 1$ only if required by the correction step (see below)). We use $\Delta \mu_{0}^{(0)}=\Delta \mu_{0}$ for some given initial step size $\Delta \mu_{0}$, whereas for $k \geq$ 1 the predicted step size $\Delta \mu_{k}^{(0)}$ is chosen by

$$
\begin{aligned}
& \Delta \mu_{k}^{(0)}:= \\
& \left(\frac{\left\|\Delta \mathbf{x}^{\left(j_{0}\right)}\left(\mu_{k}\right)\right\|}{\left\|\tilde{\mathbf{x}}\left(\mu_{k}\right)-\tilde{\mathbf{x}}^{\left(j_{0}\right)}\left(\mu_{k}\right)\right\|} \frac{\sqrt{2}-1}{2 \Theta\left(\mu_{k}\right)}\right)^{1 / 2} \Delta \mu_{k-1}
\end{aligned}
$$

Here, $\Delta \mu_{k-1}$ is the computed continuation step size, $\Delta \mathbf{x}^{\left(j_{0}\right)}\left(\mu_{k}\right)$ is the first Newton correction (see below), and $\Theta\left(\mu_{k}\right)<1$ is the contraction factor associated with a successful previous continuation step.

Corrector step: As a corrector, we use Newton's method applied to $F\left(\mathbf{x}\left(\mu_{k+1}\right), \mu_{k+1}\right)=0$ with $\tilde{\mathbf{x}}^{\left(j_{0}\right)}\left(\mu_{k+1}\right)$ from (68b) as a start vector. In particular, for $\ell \geq 0$ (Newton iteration index) and $j_{\ell} \geq 0(j$ being the steplength correction index) we compute $\Delta \mathbf{x}^{\left(j_{\ell}\right)}\left(\mu_{k+1}\right)$ according to

$$
\begin{aligned}
& F_{\mathbf{x}}\left(\tilde{\mathbf{x}}^{\left(j_{\ell}\right)}\left(\mu_{k+1}\right), \mu_{k+1}\right) \Delta \mathbf{x}^{\left(j_{\ell}\right)}\left(\mu_{k+1}\right)=(70) \\
& -F\left(\tilde{\mathbf{x}}^{\left(j_{\ell}\right)}\left(\mu_{k+1}\right), \mu_{k+1}\right),
\end{aligned}
$$

update $\tilde{\mathbf{x}}^{\left(j_{\ell+1}\right)}\left(\mu_{k+1}\right):=\tilde{\mathbf{x}}^{\left(j_{\ell}\right)}\left(\mu_{k+1}\right)+\Delta \mathbf{x}^{\left(j_{\ell}\right)}\left(\mu_{k+1}\right)$ and compute $\overline{\Delta \mathbf{x}}^{\left(j_{\ell}\right)}\left(\mu_{k+1}\right)$ as the associated simplified Newton correction

$$
\begin{aligned}
& F_{\mathbf{x}}\left(\tilde{\mathbf{x}}^{\left(j_{\ell}\right)}\left(\mu_{k+1}\right), \mu_{k+1}\right) \overline{\Delta \mathbf{x}}^{\left(j_{\ell}\right)}\left(\mu_{k+1}\right)=(71) \\
& -F\left(\tilde{\mathbf{x}}^{\left(j_{\ell}\right)}\left(\mu_{k+1}\right)+\Delta \mathbf{x}^{\left(j_{\ell}\right)}\left(\mu_{k+1}\right), \mu_{k+1}\right) .
\end{aligned}
$$

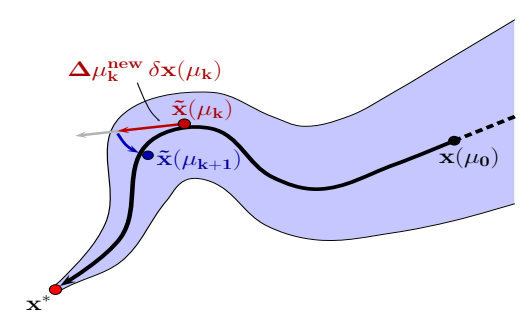

Fig. (21): Correction step of the adaptive continuation method.

Convergence of Newton's method is monitored by means of

$$
\Theta^{\left(j_{\ell}\right)}\left(\mu_{k+1}\right):=\left\|\overline{\Delta \mathbf{x}}^{\left(j_{\ell}\right)}\left(\mu_{k+1}\right)\right\| /\left\|\Delta \mathbf{x}^{\left(j_{\ell}\right)}\left(\mu_{k+1}\right)\right\| .
$$


In case of successful convergence, we set $\tilde{\mathbf{x}}\left(\mu_{k+1}\right):=\tilde{\mathbf{x}}^{\left(j_{\ell}\right)}\left(\mu_{k+1}\right)$ with $\ell$ being the current Newton iteration index, accept the current step size $\Delta \mu_{k}:=\Delta \mu_{k}^{(j)}$ with current steplength correction index $j$ and proceed with the next continuation step. However, if the monotonicity test

$$
\Theta^{\left(j_{\ell}\right)}\left(\mu_{k+1}\right)<1
$$

fails for some $j_{\ell} \geq 0$, the predicted steplength $\Delta \mu_{k}^{(j)}$ has been chosen too large so that the predicted solution $\tilde{\mathbf{x}}^{\left(j_{0}\right)}\left(\mu_{k+1}\right)$ is not situated within the Kantorovich neighborhood of $\mathbf{x}\left(\mu_{k+1}\right)$, i.e., it is outside the contraction tube around the barrier path (cf. Fig. (21)). In this case, we perform a correction of the steplength for the tangent direction $\delta \mathbf{x}\left(\mu_{k}\right)$ such that the new iterate stays within the contraction tube. To do so, the continuation step from (68b) has to be repeated with the reduced step size

$$
\begin{aligned}
\Delta \mu_{k}^{(j+1)} & :=\left(\frac{\sqrt{2}-1}{g\left(\Theta^{(j \ell)}\right)}\right)^{1 / 2} \Delta \mu_{k}^{(j)}, \\
g(\Theta) & :=\sqrt{\Theta+1}-1
\end{aligned}
$$

until we either achieve convergence or for some prespecified lower bound $\Delta \mu_{\text {min }}$ observe

$$
\Delta \mu_{k}^{(j+1)}<\Delta \mu_{\min }
$$

In the latter case, we stop the algorithm and report convergence failure.

The Newton steps are realized by an inexact Newton method featuring right-transforming iterations (cf., e.g., $[64,65])$. The derivatives occurring in the KKT conditions and the Hessians are computed by automatic differentiation (cf., e.g., [53]).

As mentioned before, one of the issues in the optimal design of the biochips is to make sure that the reservoir is filled with a very precise amount of the probe containing liquid. This is taken care of by a capillary barrier placed between a channel and the reservoir.

Since the acoustic streaming can be modeled by stationary Stokes flow, the optimal design of the capillary barriers fits the framework developed in this subsection.

As computational domain we have chosen part of a channel with a capillary barrier at its end and part of a reservoir connected with the channel by the capillary barrier. In the objective functional, we have chosen $\kappa_{1}=\kappa_{2}=1$ and $\mathbf{v}^{d}, \mathbf{p}^{d}$ based on information provided by the design engineers at the cooperating company producing the biochips. The problem has

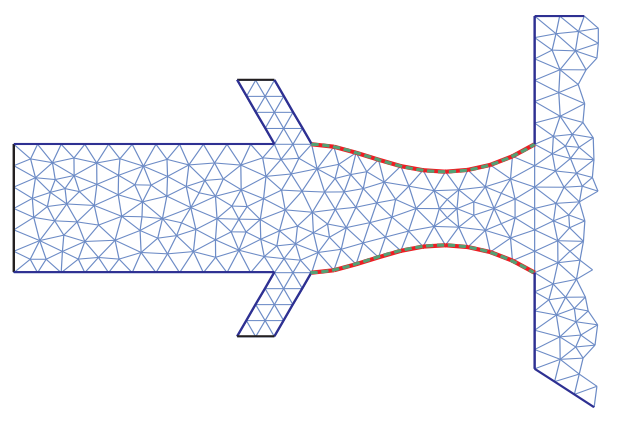

Fig. (22): Optimal shape of the capillary barrier and underlying finite element mesh

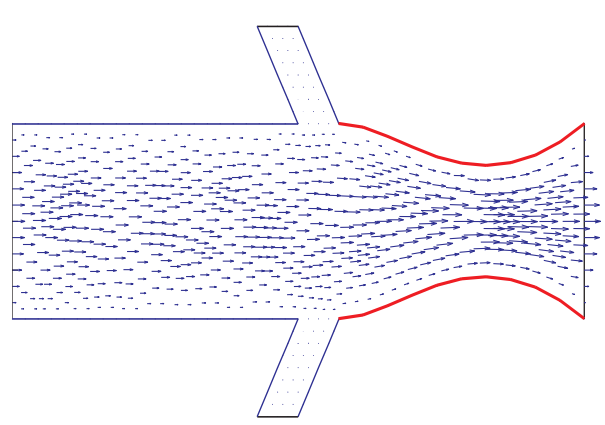

Fig. (23): Velocity field for the optimal configuration when the barrier is not in stopping mode

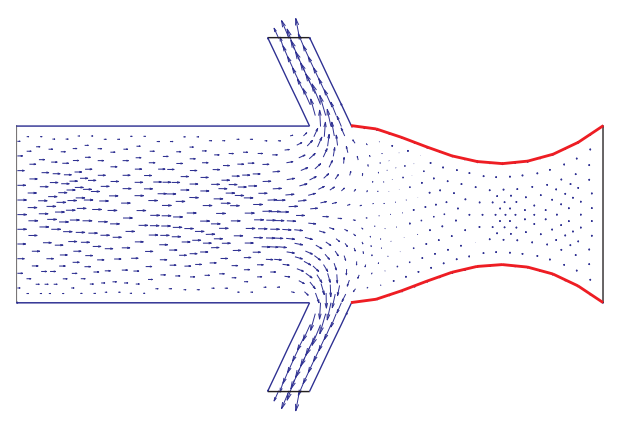

Fig. (24): Velocity field (back flow) for the optimal configuration when the barrier is in stopping mode 
been discretized by P2/P1 Taylor-Hood elements. Fig. (22) displays the computed optimal shape of the barrier together with an underlying finite element mesh. The channel additionally has passive outlet valves (cf. Fig. (22)) that are activated when the barrier operates in stopping mode and back flow occurs. Fig. (23) provides a visualization of the velocity field for the optimized channel under conditions of flow from the channel into the reservoir. Likewise, Fig. (24) displays the velocity field for the optimized channel under back flow conditions, i.e., when the capillary barrier operates in stopping mode.

Below we report the convergence history and execution time of the algorithm for a sufficiently fine finite element mesh with a total of $N_{d o f}=62916$ degrees of freedom. We used 16 Bézier control points for the Bézier curve representation of the capillary barrier as design variables and a tolerance $t o l=1.0 E-03$ as termination criterion. Table 3 displays the convergence history of the algorithm.

\begin{tabular}{|c|c|c|c|c|}
\hline$k$ & $\mu$ & $\Delta \mu$ & $\Delta J$ & Time \\
\hline 0 & $2.0 \mathrm{E}+02$ & $5.0 \mathrm{E}+02$ & - & \\
\hline 1 & $6.9 \mathrm{E}+02$ & $4.9 \mathrm{E}+02$ & $2.83 \mathrm{E}+00$ & \\
\hline 2 & $1.2 \mathrm{E}+03$ & $5.3 \mathrm{E}+02$ & $4.58 \mathrm{E}-05$ & 747 min \\
\hline
\end{tabular}

Table 3: Capillary barrier: convergence history of the adaptive continuation method

\subsection{Model Reduction}

The optimal design of structures and systems based on reduced order modeling techniques can be used to significantly decrease the computational complexity while maintaining the desired accuracy of the approximation. In particular, we consider a shape optimization problem for the time-dependent Stokes system where the design only affects a relatively small area of the computational domain. Within the context of this contribution, the optimal design of capillary barriers in SAW driven microfluidic biochips is a good example for such a scenario. We will use a combination of domain decomposition and balanced truncation model reduction (BTMR) for the reduced order modeling based optimal design.
We consider the shape optimization problem

$$
\inf _{\alpha \in K} J(\alpha):=\frac{1}{2} \int_{0}^{T} \int_{\Omega(\alpha)} \ell(v, p, x, t, \alpha) d x d t,
$$

subject to the Stokes flow

$$
\begin{array}{rlr}
\frac{\partial v}{\partial t}-v_{F} \Delta v+\nabla p=f & \text { in } Q(\alpha), \\
\nabla \cdot v=0 & \text { in } Q(\alpha),
\end{array}
$$

with appropriate boundary conditions and the initial condition

$$
v(\cdot, 0)=v^{(0)} \quad \text { in } \Omega(\alpha),
$$

where $\Omega(\alpha) \subset \mathbb{R}^{2}$ is a bounded domain depending on the design parameters $\alpha=\left(\alpha_{1}, \cdots, \alpha_{m}\right)^{T} \in K$, where $\alpha_{i}, 1 \leq i \leq m$, are the Bézier control points of a Bézier curve representation of the boundary $\partial \Omega(\alpha)$ and $K$ stands for the convex set

$$
K:=\left\{\alpha_{i} \in \mathbb{R} \mid \alpha_{i}^{\text {min }} \leq \alpha_{i} \leq \alpha_{i}^{\max }, 1 \leq i \leq m\right\},
$$

Moreover, $\ell$ is a given function of the state variables $v, p$, the independent variables $x, t$ and the design variables $\alpha$, and $v_{F}$ stands for the viscosity of the fluid.

Since balanced truncation [11, 19] applies to dynamical systems, we perform a semi-discretization in space of (74a)-(74d) by stable continuous elements such as the Taylor-Hood P2-P1 element with respect to a simplicial triangulation of the spatial domain. The semi-discrete optimization problem reads

$$
\inf _{\alpha \in K} \mathbf{J}(\alpha):=\int_{0}^{T} \ell(\mathbf{v}, \mathbf{p}, \alpha, t) d t,
$$

where the integrand $\ell$ in (75a) stems from the semidiscretization of the inner integral in (74a), and the pair $(\mathbf{v}, \mathbf{p})$ is assumed to solve the Hessenberg index 2 system

$$
\begin{aligned}
& \left(\begin{array}{cc}
\mathbf{M}(\alpha) & 0 \\
0 & 0
\end{array}\right) \frac{d}{d t}\left(\begin{array}{c}
\mathbf{v}(t) \\
\mathbf{p}(t)
\end{array}\right) \\
& =-\left(\begin{array}{cc}
\mathbf{A}(\alpha) & \mathbf{B}^{T}(\alpha) \\
\mathbf{B}(\alpha) & 0
\end{array}\right)\left(\begin{array}{l}
\mathbf{v}(t) \\
\mathbf{p}(t)
\end{array}\right) \\
& +\left(\begin{array}{c}
\mathbf{K}(\alpha) \\
\mathbf{L}(\alpha)
\end{array}\right) \mathbf{f}(t), \quad t \in(0, T], \\
& \mathbf{M}(\alpha) \mathbf{v}(0)=\mathbf{v}^{0} .
\end{aligned}
$$

Here, $\mathbf{M}(\alpha), \mathbf{A}(\alpha) \in \mathbb{R}^{n_{1} \times n_{1}}$ stand for the mass and stiffness matrices, $\mathbf{B}(\alpha) \in \mathbb{R}^{n_{2} \times n_{1}}, n_{2}<n_{1}$, refers 
to the discrete divergence operator, and $\mathbf{K}(\alpha) \in$ $\mathbb{R}^{n_{1} \times k}, \mathbf{L}(\alpha) \in \mathbb{R}^{n_{2} \times k}, \mathbf{f}(t) \in \mathbb{R}^{k}, t \in(0, T)$. A Hessenberg index 2 system is an index 2 differential algebraic system where the algebraic variable is absent from the algebraic equation.

We have the following stability result for the solution of (75b),(75c) (cf. Theorem 5.1.1 in [2]):

Theorem 5.1 Assume that $\mathbf{M} \in \mathbb{R}^{n_{1} \times n_{1}}$ is symmetric positive definite, $\mathbf{A} \in \mathbb{R}^{n_{1} \times n_{1}}$ is symmetric positive definite on $\operatorname{Ker} \mathbf{B}$, i.e.,

$$
\mathbf{v}^{T} \mathbf{A v} \geq \theta\|\mathbf{v}\|^{2} \quad, \quad \mathbf{v} \in \operatorname{Ker} \mathbf{B}
$$

$\mathbf{B} \in \mathbb{R}^{n_{2} \times n_{1}}$ has full row rank $n_{2}$ and $\mathbf{f}=\left(\mathbf{f}_{1}, \mathbf{f}_{2}\right)^{T}$. Then, there exist positive constants $C_{i}, 1 \leq i \leq 8$, such that

$$
\begin{aligned}
\|\mathbf{v}\|_{L^{2}} \leq & C_{1}\left(\left\|\mathbf{v}^{(0)}\right\|+C_{2}\left\|\mathbf{f}_{2}(0)\right\|\right)+C_{3}\left\|\mathbf{f}_{1}\right\|_{L^{2}} \\
& +C_{4}\left\|\mathbf{f}_{2}\right\|_{L^{2}} \\
\|\mathbf{p}\|_{L^{2}} \leq & C_{1} C_{5}\left(\left\|\mathbf{v}^{(0)}\right\|+C_{2}\left\|\mathbf{f}_{2}(0)\right\|\right)+C_{6}\left\|\mathbf{f}_{1}\right\|_{L^{2}} \\
& +C_{7}\left\|\mathbf{f}_{2}\right\|_{L^{2}}+C_{8}\left\|\frac{d}{d t} \mathbf{f}_{2}\right\|_{L^{2}}
\end{aligned}
$$

Here, the constants $C_{i}$ are given by

$$
\begin{aligned}
C_{1}= & \frac{\sqrt{2}\left\|\overline{\mathbf{M}}^{-1 / 2}\right\|\left\|\overline{\mathbf{M}}^{1 / 2}\right\|}{\sqrt{\theta}}, \\
C_{2}= & \left\|\mathbf{B}^{T}\left(\mathbf{B M}^{-1} \mathbf{B}^{T}\right)^{-1}\right\|, \quad C_{3}=\frac{2\left\|\overline{\mathbf{M}}^{-1}\right\|}{\theta}, \\
C_{4}= & \frac{2\left\|\overline{\mathbf{M}}^{-1}\right\|}{\theta}\left\|\mathbf{A} \mathbf{M}^{-1} \mathbf{B}^{T}\left(\mathbf{B} \mathbf{M}^{-1} \mathbf{B}^{T}\right)^{-1}\right\| \\
& +\left\|\mathbf{M}^{-1} \mathbf{B}^{T}\left(\mathbf{B} \mathbf{M}^{-1} \mathbf{B}^{T}\right)^{-1}\right\|, \\
C_{5}= & \left\|\left(\mathbf{B} \mathbf{M}^{-1} \mathbf{B}^{T}\right)^{-1} \mathbf{B} \mathbf{M}^{-1}\right\|\|\mathbf{A}\|, \\
C_{6}= & \left\|\left(\mathbf{B} \mathbf{M}^{-1} \mathbf{B}^{T}\right)^{-1} \mathbf{B} \mathbf{M}^{-1}\right\| \\
& \left(\frac{2}{\theta}\left\|\overline{\mathbf{M}}^{-1}\right\|\|\mathbf{A}\|+1\right), \\
C_{7}= & \left\|\left(\mathbf{B} \mathbf{M}^{-1} \mathbf{B}^{T}\right)^{-1} \mathbf{B} \mathbf{M}^{-1}\right\| \\
& \left\|\mathbf{A} \mathbf{M}^{-1} \mathbf{B}^{T}\left(\mathbf{B} \mathbf{M}^{-1} \mathbf{B}^{T}\right)^{-1}\right\| \\
& \left(\frac{2}{\theta}\left\|\overline{\mathbf{M}}^{-1}\right\|\|\mathbf{A}\|+1\right), \\
C_{8}= & \left\|\left(\mathbf{B} \mathbf{M}^{-1} \mathbf{B}^{T}\right)^{-1}\right\|,
\end{aligned}
$$

and $\overline{\mathbf{M}}=\Pi \mathbf{M} \Pi^{T}$, where $\Pi$ stands for the oblique projection

$$
\Pi:=\mathbf{I}-\mathbf{B}^{T}\left(\mathbf{B M}^{-1} \mathbf{B}^{T}\right)^{-1} \mathbf{B} \mathbf{M}^{-1}
$$

onto Ker $\mathbf{B}^{T}$ along $\operatorname{Im} \mathbf{B}$.
We assume that the integrand $\ell$ in (75a) is of the form

$$
\begin{aligned}
& \ell(\mathbf{v}, \mathbf{p}, \alpha, t):= \\
& \frac{1}{2}|\mathbf{C}(\alpha) \mathbf{v}(t)+\mathbf{D}(\alpha) \mathbf{p}(t)+\mathbf{F}(\alpha) \mathbf{f}(t)-\mathbf{d}(t)|^{2},
\end{aligned}
$$

where $\mathbf{C}(\alpha) \in \mathbb{R}^{q \times n_{1}}$ and $\mathbf{D}(\alpha) \in \mathbb{R}^{q \times n_{2}}$ are observation matrices, $\mathbf{F}(\alpha) \in \mathbb{R}^{q \times k}$ is a feedthrough matrix, and $\mathbf{d}(t) \in \mathbb{R}^{q}, t \in(0, T)$. For ease of notation, we drop the dependence on $\alpha$. The semi-discretized Stokes optimality system consists of the state equations

$$
\begin{aligned}
& \left(\begin{array}{cc}
\mathbf{M} & \mathbf{0} \\
\mathbf{0} & \mathbf{0}
\end{array}\right) \frac{d}{d t}\left(\begin{array}{c}
\mathbf{v}(t) \\
\mathbf{p}(t)
\end{array}\right) \\
& =-\left(\begin{array}{cc}
\mathbf{A} & \mathbf{B}^{T} \\
\mathbf{B} & \mathbf{0}
\end{array}\right)\left(\begin{array}{c}
\mathbf{v}(t) \\
\mathbf{p}(t)
\end{array}\right)+\left(\begin{array}{c}
\mathbf{K} \\
\mathbf{L}
\end{array}\right) \mathbf{f}(t) \\
& \mathbf{z}(t)=\mathbf{C v}(t)+\mathbf{D} \mathbf{p}(t)+\mathbf{F f}(t) \\
& \mathbf{M v}(0)=\mathbf{v}^{0}
\end{aligned}
$$

and the adjoint equations

$$
\begin{aligned}
& -\left(\begin{array}{cc}
\mathbf{M} & \mathbf{0} \\
\mathbf{0} & \mathbf{0}
\end{array}\right) \frac{d}{d t}\left(\begin{array}{c}
\lambda(t) \\
\kappa(t)
\end{array}\right) \\
& =-\left(\begin{array}{cc}
\mathbf{A} & \mathbf{B}^{T} \\
\mathbf{B} & \mathbf{0}
\end{array}\right)\left(\begin{array}{c}
\lambda(t) \\
\kappa(t)
\end{array}\right)+\left(\begin{array}{c}
\mathbf{C}^{T} \\
\mathbf{D}^{T}
\end{array}\right) \mathbf{z}(t) \\
& \mathbf{q}(t)=\mathbf{K}^{T} \lambda(t)+\mathbf{L}^{T} \kappa(t)+\mathbf{F}^{T} \mathbf{z}(t) \\
& \mathbf{M} \lambda(T)=\lambda^{(T)}
\end{aligned}
$$

For the realization of the balanced truncation, we compute the controllability and observability Gramians $\mathbf{P}, \mathbf{Q} \in \mathbb{R}^{n_{1} \times n_{1}}$ as the solutions of the matrix Lyapunov equations

$$
\begin{gathered}
\overline{\mathbf{A}} \mathbf{P} \overline{\mathbf{M}}+\overline{\mathbf{M}} \mathbf{P} \overline{\mathbf{A}}+\overline{\mathbf{K}} \overline{\mathbf{K}}^{T}=0, \\
\overline{\mathbf{A}} \mathbf{Q} \overline{\mathbf{M}}+\overline{\mathbf{M}} \mathbf{Q} \overline{\mathbf{A}}+\overline{\mathbf{C}}^{T} \overline{\mathbf{C}}=0,
\end{gathered}
$$

where

$$
\begin{aligned}
& \overline{\mathbf{A}}:=-\Pi \mathbf{A} \Pi^{T}, \quad \overline{\mathbf{M}}:=\Pi \mathbf{M} \Pi^{T}, \quad \overline{\mathbf{K}}:=\Pi \widetilde{\mathbf{K}} \\
& \overline{\mathbf{C}}:=\Pi \widetilde{\mathbf{C}}, \quad \widetilde{\mathbf{C}}:=\mathbf{C}-\mathbf{D}\left(\mathbf{B} \mathbf{M}^{-1} \mathbf{B}^{T}\right)^{-1} \mathbf{B} \mathbf{M}^{-1} \mathbf{A} \\
& \widetilde{\mathbf{K}}:=\mathbf{K}-\mathbf{A} \mathbf{M}^{-1} \mathbf{B}^{T}\left(\mathbf{B} \mathbf{M}^{-1} \mathbf{B}^{T}\right)^{-1} \mathbf{L} .
\end{aligned}
$$

The Lyapunov equations (83a),(83b) can be solved approximately by multishift ADI techniques (cf., e.g., $[18,54,77,86])$. We factorize $\mathbf{P}=\mathbf{U U}^{T}, \mathbf{Q}=$ $\mathbf{E E}^{T}$ and perform the singular value decomposition

$$
\mathbf{U}^{T} \mathbf{M E}=\mathbf{Z S}_{n_{1}} \mathbf{Y}^{T}, \mathbf{S}_{n_{1}}:=\operatorname{diag}\left(\sigma_{1}, \cdots, \sigma_{n_{1}}\right),
$$


where $\sigma_{i}>\sigma_{i+1}, 1 \leq i \leq n_{1}-1$. We compute $\mathbf{V}, \mathbf{W}$ according to

$$
\mathbf{V}:=\mathbf{U} \mathbf{Z}_{p} \mathbf{S}_{p}^{-1 / 2} \quad, \quad \mathbf{W}:=\mathbf{E} \mathbf{Y}_{p} \mathbf{S}_{p}^{-1 / 2}
$$

where $1 \leq p \leq n_{1}$ is chosen such that $\sigma_{p+1}<\tau \sigma_{1}$ for some threshold $\tau>0$ and $\mathbf{Y}_{p}, \mathbf{Z}_{p}$ are the matrices built by the leading $p$ columns of $\mathbf{Y}, \mathbf{Z}$.

The projection matrices satisfy

$$
\mathbf{V}=\Pi^{T} \mathbf{V} \quad, \quad \mathbf{W}=\Pi^{T} \mathbf{W} \quad, \quad \mathbf{W}^{T} \mathbf{M V}=\mathbf{I} .
$$

Multiplying the state equations by $\mathbf{W}^{T}$ and the adjoint equations by $\mathbf{V}^{T}$ results in a reduced order optimality system, where the reduced order state equations turn out to be

$$
\begin{aligned}
& \frac{d}{d t} \widehat{\mathbf{v}}_{H}(t)=-\widehat{\mathbf{A}} \widehat{\mathbf{v}}_{H}(t)+\widehat{\mathbf{K}} \mathbf{f}(t), \\
& \widehat{\mathbf{z}}(t)=\widehat{\mathbf{C}} \widehat{\mathbf{v}}_{H}(t)+\widetilde{\mathbf{F}} \mathbf{f}(t)-\widetilde{\mathbf{H}} \frac{d}{d t} \mathbf{f}(t), \\
& \widehat{\mathbf{v}}_{H}(0)=\widehat{\mathbf{v}}_{H}^{0},
\end{aligned}
$$

whereas the reduced order adjoint state equations are given according to

$$
\begin{aligned}
& -\frac{d}{d t} \widehat{\lambda}_{H}(t)=-\widehat{\mathbf{A}}^{T} \widehat{\lambda}_{H}(t)+\widehat{\mathbf{C}}^{T} \widehat{\mathbf{z}}(t), \\
& \widehat{\mathbf{q}}(t)=\widehat{\mathbf{K}}^{T} \widehat{\lambda}_{H}(t)+\widetilde{\mathbf{F}}^{T} \widehat{\mathbf{z}}(t)+\widetilde{\mathbf{H}}^{T} \frac{d}{d t} \widehat{\mathbf{z}}(t), \\
& \widehat{\lambda}_{H}(T)=\widehat{\lambda}^{(T)}
\end{aligned}
$$

with appropriately defined $\widehat{\mathbf{A}}, \widehat{\mathbf{C}}, \widetilde{\mathbf{G}}, \widetilde{\mathbf{H}}$, and $\widehat{\mathbf{K}}$. Due to the stability of $\mathbf{W}^{T} \mathbf{A V}$, the classical BTMR estimate for the error in the observations and the outputs can be shown to hold true.

Theorem 5.2 Let $\mathbf{z}(t), \mathbf{q}(t), t \in[0, T]$, and $\widehat{\mathbf{z}}(t)$, $\widehat{\mathbf{q}}(t), t \in[0, T]$, be the observations and outputs of the full order and the reduced order optimality system as given by (81b),(82b) and (86b),(87b), and let $\sigma_{i}, 1 \leq i \leq n_{1}$, be the Hankel singular values from the singular value decomposition (84). Moreover, suppose that $\mathbf{v}_{H}(0)=0$ and $\lambda_{H}(T)=0$. Then, there holds

$$
\begin{gathered}
\|\mathbf{z}-\widehat{\mathbf{z}}\|_{L^{2}} \leq 2\|\mathbf{f}\|_{L^{2}}\left(\sigma_{p+1}+\cdots+\sigma_{n_{1}}\right), \\
\|\mathbf{q}-\widehat{\mathbf{q}}\|_{L^{2}} \leq 2\|\widehat{\mathbf{z}}\|_{L^{2}}\left(\sigma_{p+1}+\cdots+\sigma_{n_{1}}\right) .
\end{gathered}
$$

Proof: We refer to section 7 in [60].

Let us now consider a domain $\Omega(\alpha)$ such that

$$
\begin{aligned}
& \overline{\Omega(\alpha)}=\bar{\Omega}_{1} \cup \overline{\Omega_{2}(\alpha)}, \quad \Omega_{1} \cap \Omega_{2}(\alpha)=\emptyset, \\
& \Gamma(\alpha):=\bar{\Omega}_{1} \cap \overline{\Omega_{2}(\alpha)},
\end{aligned}
$$

where the local area of interest is $\Omega_{2}(\alpha)$, whereas the design variables $\alpha$ do not apply to the rest $\Omega_{1}$ of the computational domain. Since the design only affects $\Omega_{2}(\alpha)$ which is assumed to be relatively small, the nonlinearity is thus restricted to that part and motivates to consider a combination of domain decomposition and BTMR. We suppose that the finescale model results from a spatial discretization by P2-P1 Taylor-Hood elements with respect to a simplicial triangulation of the computational domain which aligns with the decomposition of the spatial domain. In order to ensure that the solutions of the Stokes subdomain problems associated with $\Omega_{1}$ and $\Omega_{2}(\alpha)$ are the restrictions of the solution of the global problem to the subdomains, the subdomain pressures $\mathbf{p}_{i}, 1 \leq i \leq 2$, are split into a constant $\mathbf{p}_{0, i}$ and a pressure with zero spatial average. The latter is uniquely determined as the solution of the subdomain problem, whereas $\mathbf{p}_{0}=\left(\mathbf{p}_{0,1}, \mathbf{p}_{0,2}\right)^{T}$ is determined through the coupling of the subdomain problems via the interface. The fine-scale model is used only in the local area of interest. The rest of the domain is taken care of by a reduced order model based on balanced truncation. The objective functional

$$
\begin{aligned}
& \mathbf{J}(\mathbf{v}, \mathbf{p}, \alpha):=\mathbf{J}_{1}(\mathbf{v}, \mathbf{p})+\mathbf{J}_{2}(\mathbf{v}, \mathbf{p}, \alpha) \\
& =\int_{0}^{T}\left|\mathbf{C}_{1} \mathbf{v}_{1}(t)+\mathbf{D}_{1} \mathbf{p}_{1}(t)+\mathbf{F}_{1} \mathbf{f}(t)-\mathbf{d}(t)\right|^{2} d t \\
& \int_{0}^{T} \ell\left(\mathbf{v}_{2}(t), \mathbf{p}_{2}(t), \mathbf{v}_{\Gamma}(t), \mathbf{p}_{0}(t), t, \alpha\right) d t
\end{aligned}
$$

is assumed to consist of an objective functional $\mathbf{J}_{1}$ of tracking type for subdomain $\Omega_{1}$ and an objective functional $\mathbf{J}_{2}$ for subdomain $\Omega_{2}(\alpha)$.

Grouping the state variables according to $\mathbf{x}_{i}:=$ $\left(\mathbf{v}_{i}, \mathbf{p}_{i}\right)^{T}, 1 \leq i \leq 2$, and $\mathbf{x}_{\Gamma}:=\left(\mathbf{v}_{\Gamma}, \mathbf{p}_{0}\right)^{T}$, the semidiscretized domain decomposed Stokes system can be written in block structured form according to

$$
\begin{aligned}
& \left(\begin{array}{c}
\mathbf{P}_{1} \mathbf{x}_{1} \\
\mathbf{P}_{2}(\alpha) \mathbf{x}_{2} \\
\mathbf{P}_{\Gamma}(\alpha) \mathbf{x}_{\Gamma}
\end{array}\right):= \\
& \left(\begin{array}{ccc}
\mathbf{E}_{1} & \mathbf{0} & \mathbf{0} \\
\mathbf{0} & \mathbf{E}_{2}(\alpha) & \mathbf{0} \\
\mathbf{0} & \mathbf{0} & \mathbf{E}_{\Gamma}(\alpha)
\end{array}\right) \frac{d}{d t}\left(\begin{array}{l}
\mathbf{x}_{1} \\
\mathbf{x}_{2} \\
\mathbf{x}_{\Gamma}
\end{array}\right) \\
& +\left(\begin{array}{ccc}
\mathbf{S}_{11} & \mathbf{0} & \mathbf{S}_{1 \Gamma} \\
\mathbf{0} & \mathbf{S}_{22}(\alpha) & \mathbf{S}_{2 \Gamma}(\alpha) \\
\mathbf{S}_{1 \Gamma}^{T} & \mathbf{S}_{2 \Gamma}^{T}(\alpha) & \mathbf{S}_{\Gamma \Gamma}(\alpha)
\end{array}\right)\left(\begin{array}{l}
\mathbf{x}_{1} \\
\mathbf{x}_{2} \\
\mathbf{x}_{\Gamma}
\end{array}\right)
\end{aligned}
$$




$$
=\left(\begin{array}{c}
\mathbf{N}_{1} \\
\mathbf{N}_{2}(\alpha) \\
\mathbf{N}_{\Gamma}(\alpha)
\end{array}\right) \mathbf{f} .
$$

Here, the singular block matrices $\mathbf{E}_{1}, \mathbf{E}_{2}(\alpha)$ and $\mathbf{E}_{\Gamma}(\alpha)$ are given by

$$
\begin{aligned}
& \mathbf{E}_{1}:=\left(\begin{array}{cc}
\mathbf{M}_{1} & \mathbf{0} \\
\mathbf{0} & \mathbf{0}
\end{array}\right), \mathbf{E}_{2}(\alpha):=\left(\begin{array}{cc}
\mathbf{M}_{2}(\alpha) & \mathbf{0} \\
\mathbf{0} & \mathbf{0}
\end{array}\right), \\
& \mathbf{E}_{\Gamma}(\alpha):=\left(\begin{array}{cc}
\mathbf{M}_{\Gamma}(\alpha) & \mathbf{0} \\
\mathbf{0} & \mathbf{0}
\end{array}\right),
\end{aligned}
$$

whereas $\mathbf{S}_{11}, \mathbf{S}_{22}(\alpha)$ and $\mathbf{S}_{\Gamma \Gamma}(\alpha)$ are the Stokes matrices associated with the subdomains $\Omega_{1}, \Omega_{2}(\alpha)$ and the interface $\Gamma(\alpha)$

$$
\begin{aligned}
\mathbf{S}_{11} & :=\left(\begin{array}{cc}
\mathbf{A}_{11} & \mathbf{B}_{11}^{T} \\
\mathbf{B}_{11} & \mathbf{0}
\end{array}\right) \\
\mathbf{S}_{22}(\alpha) & :=\left(\begin{array}{cc}
\mathbf{A}_{22}(\alpha) & \mathbf{B}_{22}^{T}(\alpha) \\
\mathbf{B}_{22}(\alpha) & \mathbf{0}
\end{array}\right), \\
\mathbf{S}_{\Gamma \Gamma}(\alpha) & :=\left(\begin{array}{cc}
\mathbf{A}_{\Gamma \Gamma}(\alpha) & \mathbf{B}_{0}^{T} \\
\mathbf{B}_{0} & \mathbf{0}
\end{array}\right),
\end{aligned}
$$

and $\mathbf{S}_{1 \Gamma}, \mathbf{S}_{2 \Gamma}(\alpha)$ are of the form

$$
\begin{aligned}
\mathbf{S}_{1 \Gamma} & :=\left(\begin{array}{ll}
\mathbf{A}_{1 \Gamma} & \mathbf{0} \\
\mathbf{B}_{1 \Gamma} & \mathbf{0}
\end{array}\right), \\
\mathbf{S}_{2 \Gamma}(\alpha) & :=\left(\begin{array}{ll}
\mathbf{A}_{2 \Gamma}(\alpha) & \mathbf{0} \\
\mathbf{B}_{2 \Gamma}(\alpha) & \mathbf{0}
\end{array}\right) .
\end{aligned}
$$

Finally, $\mathbf{N}_{1}, \mathbf{N}_{2}(\alpha)$ and $\mathbf{N}_{\Gamma}(\alpha)$ are given by

$$
\begin{aligned}
& \mathbf{N}_{1}:=\left(\begin{array}{c}
\mathbf{K}_{1} \\
\mathbf{L}_{1}
\end{array}\right), \quad \mathbf{N}_{2}(\alpha):=\left(\begin{array}{c}
\mathbf{K}_{2}(\alpha) \\
\mathbf{L}_{2}(\alpha)
\end{array}\right), \\
& \mathbf{N}_{\Gamma}(\alpha):=\left(\begin{array}{c}
\mathbf{K}_{\Gamma}(\alpha) \\
\mathbf{L}_{0}(\alpha)
\end{array}\right) .
\end{aligned}
$$

Introducing Lagrange multipliers

$$
\lambda_{1}(t), \lambda_{2}(t), \lambda_{\Gamma}(t) \text { and } \kappa_{1}(t), \kappa_{2}(t), \kappa_{0}(t)
$$

and partitioning them by means of $\mu_{i}(t):=$ $\left(\lambda_{i}(t), \kappa_{i}(t)\right)^{T}, 1 \leq i \leq 2, \mu_{\Gamma}(t):=\left(\lambda_{\Gamma}(t), \kappa_{0}(t)\right)^{T}$, the Lagrangian associated with (91),(92) is given by

$$
\begin{aligned}
& \mathscr{L}(\mathbf{x}, \mu, \alpha):=\mathbf{J}(\mathbf{v}, \mathbf{p}, \alpha) \\
& +\int_{0}^{T}\left(\begin{array}{c}
\mu_{1}(t) \\
\mu_{2}(t) \\
\mu_{\Gamma}(t)
\end{array}\right) \cdot\left(\begin{array}{c}
\mathbf{P}_{1} \mathbf{x}_{1}(t)-\mathbf{N}_{1} \mathbf{f} \\
\mathbf{P}_{2}(\alpha) \mathbf{x}_{2}(t)-\mathbf{N}_{2}(\alpha) \mathbf{f} \\
\mathbf{P}_{\Gamma}(\alpha) \mathbf{x}_{\Gamma}(t)-\mathbf{N}_{\Gamma}(\alpha) \mathbf{f}
\end{array}\right) d t .
\end{aligned}
$$

The optimality conditions for the domain decomposed problem can be derived directly in terms of the derivatives of the Lagrangian $\mathscr{L}$. The optimality system with respect to the subdomain $\Omega_{1}$ is amenable to the application of BTMR. In particular, there exist projection matrices $\mathbf{V}_{1}, \mathbf{W}_{1}$ such that the reduced state equations associated with $\Omega_{1}$ are of the form

$$
\begin{aligned}
& \frac{d}{d t} \widehat{\mathbf{v}}_{1}(t)= \\
& -\mathbf{W}_{1}^{T} \mathbf{A}_{11} \mathbf{V}_{1} \widehat{\mathbf{v}}_{1}(t)+\mathbf{W}_{1}^{T} \widetilde{\mathbf{K}}_{1}\left(\begin{array}{c}
\widehat{\mathbf{v}}_{\Gamma}(t) \\
\widehat{\mathbf{p}}_{0}(t) \\
\mathbf{f}(t)
\end{array}\right) \\
& \left(\begin{array}{c}
\widehat{\mathbf{z}}_{v, \Gamma}(t) \\
\widehat{\mathbf{z}}_{p, \Gamma}(t) \\
\widehat{\mathbf{z}}_{1}(t)
\end{array}\right)=\widetilde{\mathbf{C}}_{1} \mathbf{V}_{1} \widehat{\mathbf{v}}_{1}(t) \\
& +\widetilde{\mathbf{F}}_{1}\left(\begin{array}{c}
\widehat{\mathbf{v}}_{\Gamma}(t) \\
\widehat{\mathbf{p}}_{0}(t) \\
\mathbf{f}(t)
\end{array}\right)-\widetilde{\mathbf{H}}_{1} \frac{d}{d t}\left(\begin{array}{c}
\widehat{\mathbf{v}}_{\Gamma}(t) \\
\widehat{\mathbf{p}}_{0}(t) \\
\mathbf{f}(t)
\end{array}\right)
\end{aligned}
$$

whereas the reduced adjoint state equations are given by

$$
\begin{aligned}
& -\frac{d}{d t} \widehat{\lambda}_{1}(t)= \\
& -\mathbf{V}_{1}^{T} \mathbf{A}_{11} \mathbf{W}_{1} \widehat{\lambda}_{1}(t)+\mathbf{V}_{1}^{T} \widetilde{\mathbf{C}}_{1}\left(\begin{array}{c}
\widehat{\lambda}_{1}(t) \\
\widehat{\kappa}_{0}(t) \\
-\widehat{\mathbf{z}}_{1}(t)
\end{array}\right), \\
& \left(\begin{array}{c}
\widehat{\mathbf{q}}_{v, \Gamma}(t) \\
\widehat{\mathbf{q}}_{p, \Gamma}(t) \\
\widehat{\mathbf{q}}_{1}(t)
\end{array}\right)=-\widetilde{\mathbf{B}}_{1}^{T} \mathbf{W}_{1} \widehat{\lambda}_{1}(t) \\
& +\widetilde{\mathbf{F}}_{1}^{T}\left(\begin{array}{c}
\widehat{\lambda}_{1}(t) \\
\widehat{\kappa}_{0}(t) \\
-\widehat{\mathbf{z}}_{1}(t)
\end{array}\right)+\widetilde{\mathbf{H}}_{1}^{T} \frac{d}{d t}\left(\begin{array}{c}
\widehat{\lambda}_{1}(t) \\
\widehat{\kappa}_{0}(t) \\
-\widehat{\mathbf{z}}_{1}(t)
\end{array}\right) .
\end{aligned}
$$

Since we neither apply BTMR to subdomain $\Omega_{2}(\theta)$ nor to the interface $\Gamma(\alpha)$, the corresponding state and adjoint state equations can be derived in a straightforward way. The state and the adjoint state equations associated with the subdomain $\Omega_{2}(\alpha)$ read as follows:

$$
\begin{aligned}
& \mathbf{E}_{2}(\alpha) \frac{d}{d t}\left(\begin{array}{c}
\widehat{\mathbf{v}}_{2} \\
\widehat{\mathbf{p}}_{2}
\end{array}\right)=-\mathbf{S}_{22}(\alpha)\left(\begin{array}{c}
\widehat{\mathbf{v}}_{2} \\
\widehat{\mathbf{p}}_{2}
\end{array}\right) \\
& -\mathbf{S}_{2 \Gamma}(\alpha)\left(\begin{array}{c}
\widehat{\mathbf{v}}_{\Gamma} \\
\widehat{\mathbf{p}}_{0}
\end{array}\right)+\mathbf{N}_{2}(\alpha) \mathbf{f}(t), \\
& -\mathbf{E}_{2}(\alpha) \frac{d}{d t}\left(\begin{array}{c}
\widehat{\lambda}_{2} \\
\widehat{\kappa}_{2}
\end{array}\right)= \\
& -\mathbf{S}_{22}(\alpha)\left(\begin{array}{c}
\widehat{\lambda}_{2} \\
\widehat{\kappa}_{2}
\end{array}\right)-\mathbf{S}_{2 \Gamma}(\alpha)\left(\begin{array}{c}
\widehat{\lambda}_{\Gamma} \\
\widehat{\kappa}_{0}
\end{array}\right) \\
& -\left(\begin{array}{c}
\nabla_{\widehat{\mathbf{v}}_{2}} \ell\left(\widehat{\mathbf{v}}_{2}, \widehat{\mathbf{p}}_{2}, \widehat{\mathbf{v}}_{\Gamma}, \widehat{\mathbf{p}}_{0}, t, \alpha\right) \\
\nabla_{\widehat{\mathbf{p}}_{2}} \ell\left(\widehat{\mathbf{v}}_{2}, \widehat{\mathbf{p}}_{2}, \widehat{\mathbf{v}}_{\Gamma}, \widehat{\mathbf{p}}_{0}, t, \alpha\right)
\end{array}\right) .
\end{aligned}
$$

The state and the adjoint state equations associated 
with the interface $\Gamma(\alpha)$ are given by

$$
\begin{aligned}
& \mathbf{E}_{\Gamma}(\alpha) \frac{d}{d t}\left(\begin{array}{c}
\widehat{\mathbf{v}}_{\Gamma} \\
\widehat{\mathbf{p}}_{0}
\end{array}\right)=-\mathbf{S}_{\Gamma \Gamma}(\alpha)\left(\begin{array}{c}
\widehat{\mathbf{v}}_{\Gamma} \\
\widehat{\mathbf{p}}_{0}
\end{array}\right) \\
& +\left(\begin{array}{c}
\widehat{\mathbf{z}}_{v, \Gamma} \\
\widehat{\mathbf{z}}_{p, \Gamma}
\end{array}\right)-\mathbf{S}_{2 \Gamma}^{T}(\alpha)\left(\begin{array}{c}
\widehat{\mathbf{v}}_{2} \\
\widehat{\mathbf{p}}_{2}
\end{array}\right)+\mathbf{N}_{\Gamma}(\alpha) \mathbf{f}(t), \\
& -\mathbf{E}_{\Gamma}(\alpha) \frac{d}{d t}\left(\begin{array}{c}
\widehat{\lambda}_{\Gamma} \\
\widehat{\kappa}_{0}
\end{array}\right)=-\mathbf{S}_{\Gamma \Gamma}(\alpha)\left(\begin{array}{c}
\widehat{\lambda}_{\Gamma} \\
\widehat{\kappa}_{0}
\end{array}\right) \\
& +\left(\begin{array}{c}
\widehat{\mathbf{q}}_{\mathbf{v}, \Gamma} \\
\widehat{\mathbf{q}}_{\mathbf{p}, \Gamma}
\end{array}\right)-\mathbf{S}_{2 \Gamma}^{T}(\alpha)\left(\begin{array}{c}
\widehat{\lambda}_{2}(t) \\
\widehat{\kappa}_{2}(t)
\end{array}\right) \\
& -\left(\begin{array}{c}
\nabla_{\widehat{\mathbf{v}}_{\Gamma}} \ell\left(\widehat{\mathbf{v}}_{2}, \widehat{\mathbf{p}}_{2}, \widehat{\mathbf{v}}_{\Gamma}, \widehat{\mathbf{p}}_{0}, t, \alpha\right) \\
\nabla_{\widehat{\mathbf{p}}_{0}} \ell\left(\widehat{\mathbf{v}}_{2}, \widehat{\mathbf{p}}_{2}, \widehat{\mathbf{v}}_{\Gamma}, \widehat{\mathbf{p}}_{0}, t, \alpha\right)
\end{array}\right) .
\end{aligned}
$$

The equations (96a),(96b) and (97a),(97b) are complemented by the variational inequality

$$
\begin{aligned}
& \int_{0}^{T} \nabla_{\alpha} \ell\left(\mathbf{v}_{2}, \mathbf{p}_{2}, \mathbf{v}_{\Gamma}, \mathbf{p}_{0}, t, \alpha\right) d t \\
& +\int_{0}^{T}\left(\begin{array}{c}
\widehat{\mu}_{2}(t) \\
\widehat{\mu}_{\Gamma}(t)
\end{array}\right)^{T}\left(\begin{array}{c}
\left(\mathbf{D}_{\alpha} \mathbf{P}_{2}(\alpha)(\tilde{\alpha}-\alpha)\right) \widehat{\mathbf{x}}_{2}(t) \\
\left(\mathbf{D}_{\alpha} \mathbf{P}_{\Gamma}(\alpha)(\tilde{\alpha}-\alpha)\right) \widehat{\mathbf{x}}_{\Gamma}(t)
\end{array}\right. \\
& \left.-\begin{array}{l}
\left(\mathbf{D}_{\alpha} \mathbf{N}_{2}(\alpha)(\tilde{\alpha}-\alpha)\right) \mathbf{f}(t) \\
\left(\mathbf{D}_{\alpha} \mathbf{N}_{\Gamma}(\alpha)(\tilde{\alpha}-\alpha)\right) \mathbf{f}(t)
\end{array}\right) d t \geq 0
\end{aligned}
$$

which is supposed to hold true for all $\tilde{\alpha} \in K$. Here, $\widehat{\mathbf{x}}_{2}:=\left(\widehat{\mathbf{v}}_{2}, \widehat{\mathbf{p}}_{2}\right), \widehat{\mathbf{x}}_{\Gamma}:=\left(\widehat{\mathbf{v}}_{\Gamma}, \widehat{\mathbf{p}}_{0}\right)$. Moreover, $\mathbf{N}_{2}(\alpha), \mathbf{N}_{\Gamma}(\alpha)$ and $\mathbf{P}_{2}(\alpha), \mathbf{P}_{\Gamma}(\alpha)$ are given by as in (92).

Under some assumptions on the data of the shape optimization problem under consideration we can establish an upper bound for the error in the optimal design between the full order and the reduced order model.

(A) There exists a constant $\gamma>0$ such that for all $\widehat{\alpha}, \alpha \in K$ there holds

$$
(\nabla \widehat{\mathbf{J}}(\widehat{\alpha})-\nabla \mathbf{J}(\alpha))^{T}(\widehat{\alpha}-\alpha) \geq \gamma\|\widehat{\alpha}-\alpha\|^{2} .
$$

(A $\left.\mathbf{A}_{2}\right)$ The objective functional $\mathbf{J}_{1}$ does not explicitly depend on the pressure, i.e., it is supposed to be of the form

$$
\mathbf{J}_{1}\left(\mathbf{v}_{1}\right)=\frac{1}{2} \int_{0}^{T}\left|\mathbf{C}_{1} \mathbf{v}_{1}(t)+\mathbf{F}_{1} \mathbf{f}(t)-\mathbf{d}(t)\right|^{2} d t .
$$

(A) Treating the states $\mathbf{x}_{2}:=\left(\mathbf{v}_{2}, \mathbf{p}_{2}\right)^{T}$ and $\mathbf{x}_{\Gamma}:=$ $\left(\mathbf{v}_{\Gamma}, \mathbf{p}_{0}\right)^{T}$ in the integrand $\ell$ of the objective functional

$$
\mathbf{J}_{2}\left(\mathbf{x}_{2}, \mathbf{x}_{\Gamma}, \alpha\right)=\frac{1}{2} \int_{0}^{T} \ell\left(\mathbf{x}_{2}, \mathbf{x}_{\Gamma}, t, \alpha\right) d t,
$$

as implicit functions of $\alpha$, we assume that for some positive constant $L_{1}$ the Lipschitz condition

$$
\begin{aligned}
& \left\|\nabla_{\alpha} \ell\left(\mathbf{x}_{2}, \mathbf{x}_{\Gamma}, t, \alpha\right)-\nabla_{\alpha} \ell\left(\mathbf{x}_{2}^{\prime}, \mathbf{x}_{\Gamma}^{\prime}, t, \alpha\right)\right\| \leq \\
& L_{1}\left(\left\|\mathbf{x}_{2}-\mathbf{x}_{2}^{\prime}\right\|^{2}+\left\|\mathbf{x}_{\Gamma}-\mathbf{x}_{\Gamma}^{\prime}\right\|^{2}\right)^{1 / 2}
\end{aligned}
$$

is satisfied uniformly in $\alpha \in K$ and $t \in[0, T]$.

(A) There exists a constant $L_{2}>0$ such that for all $\alpha \in K$ and all $\alpha^{\prime}$ with $\left\|\alpha^{\prime}\right\| \leq 1$ there holds

$$
\begin{aligned}
& \max \left\{\left\|\mathbf{D}_{\alpha} \mathbf{P}_{2}(\alpha) \alpha^{\prime}\right\|,\left\|\mathbf{D}_{\alpha} \mathbf{P}_{\Gamma}(\alpha) \alpha^{\prime}\right\|,\right. \\
& \left.\left\|\mathbf{D}_{\alpha} \mathbf{N}_{2}(\alpha) \alpha^{\prime}\right\|,\left\|\mathbf{D}_{\alpha} \mathbf{N}_{\Gamma}(\alpha) \alpha^{\prime}\right\|\right\} \leq L_{2}
\end{aligned}
$$

(A5) The matrix $\mathbf{A}(\alpha) \in \mathbb{R}^{n_{1} \times n_{1}}$ is symmetric positive definite and $\mathbf{B}(\alpha) \in \mathbb{R}^{n_{2} \times n_{1}}$ has rank $n_{2}$. The generalized eigenvalues of $(\mathbf{A}(\alpha), \mathbf{M}(\alpha))$ have positive real part. $\mathbf{A}_{11}(\alpha) \in \mathbb{R}^{n_{11} \times n_{11}}$ is symmetric positive definite and $\mathbf{B}_{11}(\alpha) \in \mathbb{R}^{n_{21} \times n_{11}}$ has rank $n_{21}$. The generalized eigenvalues of $\left(\mathbf{A}_{11}(\alpha), \mathbf{M}_{11}(\alpha)\right)$ have positive real part.

Theorem 5.3 Under assumptions $\left(\mathbf{A}_{\mathbf{1}}\right)-\left(\mathbf{A}_{5}\right)$ let $\alpha^{*}$ and $\widehat{\alpha}^{*}$ be the optimal designs obtained by the solution of the full order and the reduced order optimization problem. Then, there exists $C>0$ such that

$$
\left\|\alpha^{*}-\widehat{\alpha}^{*}\right\| \leq \frac{C}{\gamma}\left(\sigma_{p+1}+\cdots+\sigma_{n_{1}}\right),
$$

where

$$
\begin{aligned}
& C=\left\{T L_{1}+\gamma\left\|\left(\begin{array}{c}
\widehat{\mu}_{2} \\
\widehat{\mu}_{\Gamma}
\end{array}\right)\right\|\right. \\
& \left.+L_{2}\left(c_{\lambda}+c_{\kappa}\right)\left(\sigma_{p+1}+\cdots+\sigma_{n_{1}}\right)\right\}\left(c_{v}+c_{p}\right) \\
& +L_{2}\left(c_{\lambda}+c_{\kappa}\right)\left\{\left\|\left(\begin{array}{c}
\widehat{\mathbf{x}}_{2} \\
\widehat{\mathbf{x}}_{\Gamma}
\end{array}\right)\right\|+\|\mathbf{f}\|\right\} .
\end{aligned}
$$

Here, $c_{\nu}, c_{p}, c_{\lambda}, c_{\kappa}$ are positive constants.

Proof: The assertion follows from Theorem 5.1 and Theorem 5.2 in conjunction with the assumptions $\left(\mathbf{A}_{\mathbf{1}}\right)-\left(\mathbf{\mathbf { A } _ { 5 }}\right)$. For details we refer to Lemma 5.5.1-2, Theorem 5.5.3 and Corollary 5.5.4 in [2].

Remark 5.4 In general, we cannot expect the strict convexity assumption $\left(\mathbf{A}_{\mathbf{1}}\right)$ to hold true globally, since the dependence of the objective functional on 
the design variable $\alpha$ typically is nonconvex such that there is a wide variety of critical points. However, we may assume the validity of $\left(\mathbf{A}_{\mathbf{1}}\right)$ in some neighborhood $\mathscr{U}\left(\alpha^{*}\right)$ of a local minimum $\alpha^{*} \in K$. In this sense, the result of Theorem 5.3 has to be understood as a local convergence result.

Remark 5.5 The constant $C$ in Theorem 5.3 depends on quantities like $\theta$ in (76), the derivative of $\mathbf{A}(\alpha)$ with respect to $\alpha$, etc., which in turn depends on the viscosity $v_{F}$ of the fluid. Therefore, one might have to choose a smaller truncation level $\tau>0$ for the balanced truncation model reduction $\sigma_{p+1}<\tau \sigma_{1}$.
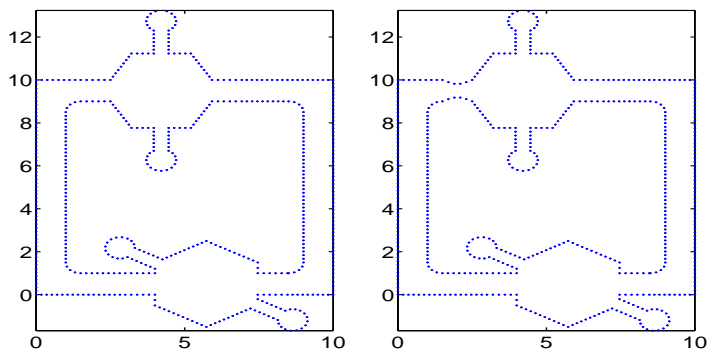

Fig. (25): Reference domain $\Omega_{\text {ref }}$ (left) and optimal domain (right).

We have considered Stokes flow in a network of microchannels and reservoirs on top of a microfluidic biochip with capillary barriers between the channels and the reservoirs to guarantee a precise filling of the reservoirs with the DNA or protein probes. The objective was twofold: Firstly, we wanted to design the walls of the barriers in such a way that a desired velocity profile $\mathbf{v}^{d}$ is attained and secondly, we wanted to minimize the vorticity $\nabla \wedge \mathbf{v}$ in subdomain $\Omega_{\text {obs }}$ of the network. The chosen computational domain $\Omega \subset \mathbb{R}^{2}$ is displayed in Figure (25) (left). It has been decomposed into subdomains $\Omega_{1}=\Omega \backslash \Omega_{2}$, and $\Omega_{2}=(1.5,2.5) \times(9,10)$ and boundaries $\Gamma_{\text {in }}=\{0\} \times(9,10), \Gamma_{\text {out }}=\{10\} \times(0,1)$, and $\Gamma_{\text {lat }}=\partial \Omega \backslash\left(\Gamma_{\text {in }} \cup \Gamma_{\text {out }}\right)$ (units in $\left.10^{-3}\right)$. The data of the problem have been chosen according to $\mathbf{f}=0$ in $\Omega \times(0, T)$, a Poiseuille velocity profile $\mathbf{v}_{\text {in }}\left(\left(x_{1}, x_{2}\right), t\right)=4\left(x_{2}-9\right)\left(10-x_{2}\right)\left(1-\frac{0.8}{15} t\right) \sin (\mathrm{t})$ on $\Gamma_{\text {in }} \times(0, T)$, outflow boundary conditions on $\Gamma_{\text {out }} \times(0, T)$, and no-slip conditions on $\Gamma_{\text {lat }} \times(0, T)$. We have used a parametrization $\Omega_{2}(\alpha)$ of $\Omega_{2}$ by means of Bézier control points $\alpha \in \mathbb{R}^{m}, m=m_{T}+$ $m_{B}$, of Bézier curve representations of $\Gamma_{2, T}$ and $\Gamma_{2, B}$, where $m_{T}$ and $m_{B}$ refer to the number of control points for $\Gamma_{2, T}$ and $\Gamma_{2, B}$, respectively. The follow- ing shape optimization problem had to be solved

$$
\begin{aligned}
J(\alpha)= & \int_{0}^{T} \int_{\Omega_{\mathrm{obs}}}|\nabla \wedge \mathbf{v}(x, t)|^{2} d x d t \\
& +\int_{0}^{T} \int_{\Omega_{2}(\alpha)}\left|\mathbf{v}(x, t)-\mathbf{v}^{d}(x, t)\right|^{2} d x d t,
\end{aligned}
$$

subject to the Stokes equations (74b)-(74d) with $T=15, v=1 / 50, \mathbf{v}^{(0)}=\mathbf{0}$ and the constraints $\alpha_{i}^{\text {min }} \leq \alpha_{i} \leq \alpha_{i}^{\text {max }}, 1 \leq i \leq m$, where the bounds $\alpha_{i}^{\min }, \alpha_{i}^{\max }$ on the design variables have been chosen such that the constraints were never active in this example. We have selected $m_{T}=6, m_{B}=$ 6 Bézier control points for the top and the bottom boundary of $\Omega_{2}(\alpha)$ with the respective first and last control points being fixed. The desired velocity $\mathbf{v}^{d}$ has been chosen as the restriction to $\Omega_{2}(\alpha)$ of the velocity computed on $\Omega(\widetilde{\alpha})$ for $\widetilde{\alpha}=$ $(9.9,9.75,9.75,9.9,9.1,9.25,9.25,9.1)$. For spatial discretization, we have used P2-P1 Taylor-Hood elements with respect to simplicial triangulations $\mathscr{T}_{h}(\Omega)$ of different granularity aligning with the decomposition into $\Omega_{1}$ and $\Omega_{2}$. The Lyapunov equations have been solved by a multishift ADI method with four shifts that have been computed as in [60]. For the model reduction, we have selected all Hankel singular values $\sigma_{j}$ with $\sigma_{j} \geq 10^{-3} \sigma_{1}$. Further, we have used automatic differentiation [53] to compute the derivatives with respect to the design variables $\alpha$ and we have solved the discretized optimization problems by a projected BFGS method with Armijo line search. The optimization algorithm has been terminated when the norm of the projected gradient was less than $10^{-4}$.

The results in Figure (25)(right) and Figure (26)(27) and Table 5 have been generated with respect to the finest mesh with a total of $N_{\mathbf{v}, \text { dof }}=16806 \mathrm{de}$ grees of freedom. In particular, the computed optimal domain $\Omega\left(\alpha^{*}\right)$ is shown in Figure (25) (right). We note that the optimal shape $\alpha^{*}$ is close, but not equal to $\widetilde{\alpha}$, since the objective $J(\alpha)$ also includes the term $\int_{\Omega_{\mathrm{obs}}}|\nabla \wedge \mathbf{v}(x, t)|^{2} d x$.

Fig. (26) (left) shows the convergence history of the multishift ADI in terms of the normalized residuals for the approximate solution of the controllability and observability Lyapunov equations, whereas Fig. (26) (right) displays the largest Hankel singular values with respect to the threshold $10^{-3} \sigma_{1}$ (indicated by the solid line). In this case, twenty-nine Hankel singular values and corresponding singular vectors have been chosen to determine the reduced 

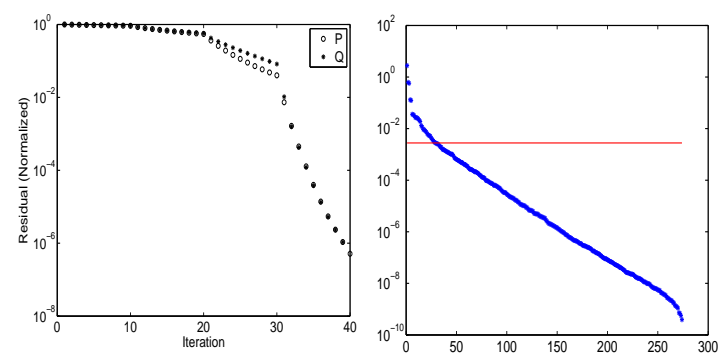

Fig. (26): Normalized residuals generated by the multishift ADI for the approximate solution of the controllability Lyapunov equation $(o)$ and of the observability Lyapunov equation $(*)$ (left). Largest Hankel singular values and the threshold $10^{-3} \sigma_{1}$ (right).

order model.
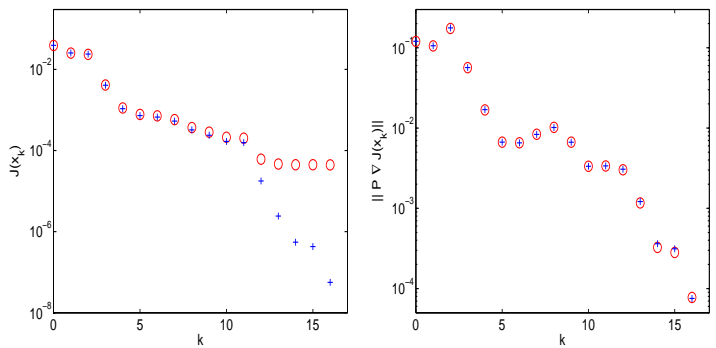

Fig. (27): Convergence history of the objective functionals for the full (+) and reduced (o) order model (left) and convergence history of the projected gradients for the full (+) and reduced (o) order model (right).

The convergence histories of the projected BFGS algorithm are shown in Fig. (27). We note that except for the final iterations, the convergence behavior of the optimization algorithm is nearly identical. This can be explained as follows: The DDBTMR approach approximates the objective function as well as the gradient of the full order problem with an absolute error that is proportional to the sum of the truncated singular values. The objective function values begin to differ when the full order objective function value drops below $10^{-4}$. However, we have stopped the iteration when the gradient norms were less than $10^{-4}$. If we had required a stricter stopping criteria, then the gradient norms between the full and the reduced order model would have started to differ as well.

Table 4 displays the sizes of the reduced and the full order problems (in Degrees of Freedom (DoF)) for an initial coarse grid and three levels of refine-

\begin{tabular}{|c||c|c|c|c|c|}
\hline $\begin{array}{c}\text { grid } \\
\text { number }\end{array}$ & $q$ & $N_{\mathbf{v}, \text { dof }}^{(1)}$ & $N_{\widehat{\mathbf{v}}, \text { dof }}^{(1)}$ & $N_{\mathbf{v}, \text { dof }}$ & $N_{\widehat{\mathbf{v}}, \text { dof }}$ \\
\hline 1 & 149 & 4752 & 23 & 4862 & 133 \\
2 & 313 & 7410 & 25 & 7568 & 183 \\
3 & 361 & 11474 & 26 & 11700 & 252 \\
4 & 537 & 16472 & 29 & 16806 & 363 \\
\hline
\end{tabular}

Table 4: The number $q$ of observations in $\Omega_{1}$, the numbers $N_{\mathbf{v}, \text { dof }}^{(1)}, N_{\widehat{\mathbf{v}}, \text { dof }}^{(1)}$ of velocity DoF in $\Omega_{1}$ (full and reduced order), and the numbers $N_{\mathbf{v}, \text { dof }}, N_{\widehat{\mathbf{v}}}$,dof of velocity DoF in $\Omega$ (full and reduced order) for four meshes.

\begin{tabular}{|c|c|c|c|}
\hline$\alpha^{*}$ & $\widehat{\alpha}^{*}$ & $\alpha^{*}$ & $\widehat{\alpha}^{*}$ \\
\hline 9.8987 & 9.9026 & 9.7510 & 9.7498 \\
9.7496 & 9.7484 & 9.8994 & 9.9021 \\
9.0991 & 9.0940 & 9.2499 & 9.2514 \\
9.2504 & 9.2511 & 9.0989 & 9.0956 \\
\hline
\end{tabular}

Table 5: Optimal shape parameters $\alpha^{*}$ and $\widehat{\alpha}^{*}$ (rounded to 5 digits) computed by minimizing the full and the reduced order model

ment. We observe that the size of the reduced order model is nearly independent of the grid size and that the model reduction achieved by BTMR on the linear subproblem corresponding to $\Omega_{1}$ is substantial. Finally, the optimal shapes $\alpha^{*}$ and $\widehat{\alpha}^{*}$ for the full and the reduced order model are shown in Table 5. For the finest grid problem, the error between the full and the reduced order model solution is $\left\|\alpha^{*}-\widehat{\alpha}^{*}\right\|_{2}=8.0751 \cdot 10^{-3}$.

Acknowledgments. The authors acknowledge support by the NSF under Grant No. DMS-0707602, DMS-0810176, DMS-0914788, by the German National Science Foundation within the Priority Programs SPP 1253, SPP 1506, by the German Federal Ministry for Education and Research (BMBF) within the projects BMBF-FROPT and BMBFMeFreSim, and by the European Science Foundation (ESF) within the ESF Program OPTPDE. The second author also wants to express his sincere thanks to the IMA, University of Minnesota, for its hospitality during his stay in Fall 2010 on occasion of the IMA Special Year 'Simulating our complex world: modeling, computation, and analysis. 


\section{REFERENCES}

[1] G. Allaire. Shape Optimization by the Homogenization Method. Springer, Berlin-Heidelberg-New York, 2002

[2] H. Antil. Optimization and Model Reduction of Time Dependent PDE-Constrained Optimization Problems: Applications to Acoustic Wave Drivern Microfluidic Biochips. Ph.D. Thesis, Department of Mathematics, University of Houston, Houston, TX, 2009.

[3] H. Antil, A. Gantner, R.H.W. Hoppe, D. Köster, K.G. Siebert, and A. Wixforth. Modeling and simulation of piezoelectrically agitated acoustic streaming on microfluidic biochips. In: Proc. 17th Int. Conf. on Domain Decomposition Methods (Langer, U. et al.; eds.), Lecture Notes in Computational Science and Engineering, Vol. 60, pp. 305-312, Springer, Berlin Heidelberg-New York, 2007.

[4] H. Antil, R. Glowinski, R.H.W. Hoppe, C. Linsenmann, T.W. Pan, and A. Wixforth. Modeling, simulation, and optimization of surface acoustic wave driven microfluidic biochips. J. Comp. Math 28, 149-169, 2010

[5] H. Antil, R.H.W. Hoppe, and C. Linsenmann. Path-following primal-dual interior-point methods for shape optimization of stationary flow problems. J. Numer. Math., 11, 81-100, 2007.

[6] H. Antil, M. Heinkenschloss, and R. H. W. Hoppe. Domain decomposition and balanced truncation model reduction for shape optimization of the Stokes system. Optimization Methods \& Software, DOI: 10.1080/10556781003767904, 2010.

[7] H. Antil, M. Heinkenschloss, R.H.W. Hoppe, and D. Sorensen. Domain decomposition and model reduction for the numerical solution of PDE constrained optimization problems with localized optimization variables. to appear in Comp. Visual. Sci., 2010.

[8] H. Antil, M. Heinkenschloss, R. H.W. Hoppe, C. Linsenmann, and A. Wixforth. Reduced order modeling based shape optimization of surface acoustic wave driven microfluidic biochips. submitted to Math. Comput. Simul., 2010.

[9] H. Antil, R.H.W. Hoppe, and C. Linsenmann. Optimal design of stationary flow problems by path-following interior-point methods. Control \& Cybernetics, 37, 771-796, 2008.

[10] H. Antil, R.H.W. Hoppe, and C. Linsenmann. Adaptive multilevel interior-point methods in PDE constrained optimization. In Proc. 18th Int. Conf. on Domain Decomposition Methods and Applications (Bercovier, M. et al.; eds.), pp. 15-26, Lecture Notes in Computational Science and Engineering, Vol. 70, Springer, Berlin Heidelberg-New York, 2009.

[11] A. C. Antoulas. Approximation of Large-Scale Systems. SIAM Philadelphia, 2005.

[12] P. Armand, J. Benoist, and D. Orban. Dynamic updates of the barrier parameter in primal-dual methods for nonlinear programming. Computational Optimization and Applications, 41, 1-25, 2008.

[13] Z. Bai, P. Dewilde, and R. Freund. Reduced order modeling. In: Handbook of Numerical Analysis, Vol. XIII (W. Schilder and E.J.W. ter Maten; eds.), pp. 825-895, North-Holland/Elsevier, Amsterdam, 2005

[14] R.E. Bank, B.D. Welfert, and H. Yserentant. A class of iterative methods for solving saddle point problems. Numer. Math., bf 56 645-666, 1990.

[15] K.J. Bathe and E. Wilson. Numerical Methods in Finite Element Analysis. Prentice Hall, Englewood Cliffs, 1976.

[16] M.P. Bendsøe. Optimization of Structural Topology, Shape, and Material. Springer, Berlin-Heidelberg-New York, 1995.

[17] P. Benner, R.W. Freund, D. Sorensen, and A. Varga (eds.). Special issue on 'order reduction of large-scale systems'. Linear Algebra and its Applications $\mathbf{4 1 5}(2-3), 2006$.

[18] P. Benner, J.-R. Li, and T. Penzl. Numerical solution of large Lyapunov equations, Riccati equations, and linear-quadratic control problems. Numer. Lin. Alg. Appl., 15, 755-777, 2008.

[19] P. Benner, V. Mehrmann, and D. C. Sorensen (eds.). Dimension Reduction of Large-Scale Systems. Lecture Notes in Computational Science and Engineering, Vol. 45, Springer, BerlinHeidelberg-New York, 2005

[20] M. Benzi and G.H. Golub. A preconditioner for generalized saddle point problems. SIAM J. Matrix Anal. Appl., 26, 20-41, 2004.

[21] G. Biros and O. Ghattas. Parallel Lagrange-Newton-Krylov-Schur methods for PDE-constrained optimization. part i: the KrylovSchur solver. SIAM J. Sci. Comp., 27, 687-713, 2005.

[22] G. Biros and O. Ghattas. Parallel Lagrange-Newton-Krylov-Schur methods for PDE-constrained optimization. part ii: the LagrangeNewton solver and its application to optimal control of steady viscous flows. SIAM J. Sci. Comp., 27, 714-739, 2005.

[23] J.H. Bramble, J.E. Pasciak, and A.T. Vassilev. Analysis of the inexact Uzawa algorithm for saddle point problems. SIAM J. Numer. Anal., 34, 1072-1092, 1997.
[24] J.H. Bramble, J.E. Pasciak, and J. Xu. Parallel multilevel preconditioners. Math. Comp., 55, 1-22, 1990.

[25] F. Brezzi and M. Fortin. Mixed and Hybrid Finite Element Methods. Springer, Berlin-Heidelberg-New York, 1991.

[26] T. Bui-Thanh, K. Willcox, and O. Ghattas. Model reduction for large-scale systems with high-dimensional parametric input space. SIAM J. Sci. Comp. 30, 3270-3288, 2008.

[27] T. Bui-Thanh, K. Willcox, O. Ghattas, and B. van Bloemen Wanders. Goal-oriented, model-constrained optimization for reduction of large-scale systems. J. Comp. Phys. 224, 880-896, 2007.

[28] R.H. Byrd, J.C. Gilbert, and J. Nocedal. A trust region method based on interior point techniques for nonlinear programming. Math. Programming, 89, 149-185, 2000.

[29] C. Campbell. Surface Acoustic Wave Devices and Their Signal Processing Applications. Academic Press, San Diego, 1989.

[30] Z.H. Cao. Fast Uzawa algorithm for generalized saddle point problems. Appl. Numer. Math., 46, 157-171, 2003.

[31] A. Cherkaev. Variational Methods for Structural Optimization. Springer, New York, 2000.

[32] P. Ciarlet, Jr., J. Huang, and J. Zou. Some observations on generalized saddle-point problems. SIAM J. Matrix Anal. Appl., 25, 224-236, 2003.

[33] M.C. Delfour and J.P. Zolesio. Shapes and Geometries: Analysis, Differential Calculus and Optimization. SIAM, Philadelphia, 2001.

[34] P. Deuflhard. Newton Methods for Nonlinear Problems. Affine Invariance and Adaptive Algorithms. Springer, Berlin-HeidlebergNew York, 2004.

[35] G. E. Dullerud and F. Paganini. A Course in Robust Control Theory. Texts in Applied Mathematics, Vol. 36, Springer, BerlinHeidelberg-New York, 2000.

[36] A.S. El-Bakry, R.A. Tapia, T. Tsuchiya, and Y. Zhang. On the formulation and theory of the Newton interior-point method for nonlinear programming. Journal of Optimization Theory and Applications, 89, 507-541, 1996.

[37] H.C. Elman. Preconditioners for saddle point problems arising in computational fluid dynamics. Appl. Numer. Math. 43, 75-89, 2002.

[38] H.C. Elman and G.H. Golub. Inexact and preconditioned Uzawa algorithms for saddle point problems. SIAM J. Numer. Anal., 6 , 1645-1661, 1994.

[39] G. Endoh, K. Hashimoto, and M. Yamaguchi. Surface acoustic wave propagation characterization by finite-element method and spectral domain analysis. Jpn. J. Appl. Phys. 34, 5B, 2632-2637, 1995.

[40] A.C. Eringen and G.A. Maugin. Electrodynamics of Continua I. Foundations and Solid Media. Springer, Berlin-Heidelberg-New York, 1990.

[41] J. Fagerholm. Wave propagation in diffractive optical elements and surface-acoustic wave devices. CSC Research Report R07/96, Center for Scientific Computing, Helsinki University of Technology, Espoo, 1996

[42] M. Fahl and E. Sachs. Reduced order modelling approaches to PDE-constrained optimization based on proper orthogonal decomposition. In: Large-Scale PDE-Constrained Optimization (L.T. Biegler et al.; eds.), Lect. Notes in Comput. Sci. Engrg., Vol. 30, Springer, Berlin-Heidelberg-New York, 2003.

[43] A.V. Fiacco and G.P. McCormick. Nonlinear Programming: Sequential Unconstrained Minimization Techniques. SIAM, Philadelphia, 1990.

[44] A. Forsgren, Ph.E. Gill, and M.H. Wright. Interior methods for nonlinear optimization. SIAM Rev. 44, 522-597, 2002

[45] T. Franke and A. Wixforth. Microfluidics for miniaturized laboratories on a chip. ChemPhysChem, 9, 2140-2156, 2008.

[46] R.W. Freund. Model reduction methods based on Krylov subspaces. Acta Numerica, 267-319, 2003.

[47] A. Gantner, R.H.W. Hoppe, D. Köster, K.G. Siebert, and A. Wixforth. Numerical simulation of piezoelectrically agitated surface acoustic waves on microfluidic biochips. Comp. Visual. Sci., 10, 145-161, 2007

[48] D.M. Gay, M.I. Overton, and M.H. Wright. Primal-dual interior method for nonconvex nonlinear programming. In: Advances in Nonlinear Programming (Y. Yuan; ed.), pp. 31-56, Kluwer, Dordrecht, 1998.

[49] K. Glover. All optimal Hankel-norm approximations of linear multivariable systems and their $L^{\infty}$-error bounds. Internat. J. Control, 39, 1115-1193, 1984.

[50] R. Glowinski. Handbook of Numerical Analysis: Numerical Methods for Fluids. Elsevier, Amsterdam, 2004 
[51] M.A. Grepl and A.T. Patera. A posteriori error bounds for reducedbasis approximations of parametrized parabolic partial differential equations. ESAIM: M2AN, 39, 157-181, 2005.

[52] M.A. Grepl, Y. Maday, N.C. Nguyen, and A.T. Patera. Efficient reduced-basis treatment of nonaffine and nonlinear partial differential equations. ESAIM: M2AN, 41, 575-605, 2007.

[53] A. Griewank and A. Walther. Evaluating Derivatives. Principles and Techniques of Algorithmic Differentiation. 2nd Edition. SIAM, Philadelphia, 2008.

[54] S. Gugercin, D.C. Sorensen, and A.C. Antoulas. A modified lowrank Smith method for large scale Lyapunov equations. Numer. Algorithms, 32, 27-ï ; 55, 2003.

[55] M.D. Gunzburger. Perspectives in Flow Control and Optimization. SIAM, Philadelphia, 2003

[56] Z. Guttenberg, H. Müller, H. Habermüller, A. Geisbauer, J. Pipper, J. Felbel, M. Kilepinski, J. Scriba, and A. Wixforth. Plana chip device for PCR and hybridization with surface acoustic wave pump. Lab on a Chip, 5, 308-317, 2005

[57] K. Hasegawa and M. Koshiba. Finite-element solution of Rayleigh-wave scattering from reflective gratings on a piezoelectric substrate. IEEE Trans. Ultrason. Ferroelectr. Freq. Contr. 37, 99-105, 1990

[58] J. Haslinger and R.A.E. Mäkinen. Introduction to Shape Optimization: Theory, Approximation, and Computation. SIAM, Philadelphia, 2004.

[59] J. Haslinger and P. Neittaanmäki. Finite Element Approximation for Optimal Shape Design: Theory and Applications. John Wiley \& Sons, Chichester, 1988

[60] M. Heinkenschloss, D. C. Sorensen, and K. Sun. Balanced truncation model reduction for a class of descriptor systems with application to the Oseen equations. SIAM Journal on Scientific Computing, 30, 1038-1063, 2008.

[61] M. Hofer, M. Jungwirth, R. Lerch, and R. Weigel. Accurate and efficient modeling of SAW structures. Frequenz, 55, 64-72, 2001

[62] M. Hofer, R. Lerch, N. Finger, G. Kovacs, J. Schöberl, and U. Langer. Finite element calculations of wave propagation and excitation in periodic piezoelectric systems. In: Proc. WCCM V, 5th World Congr. on Comput. Mech., Vienna, 2002.

[63] R.H.W. Hoppe, C. Linsenmann, and H. Antil. Adaptive path following primal dual interior point methods for shape optimization of linear and nonlinear Stokes flow problems. In: Lecture Notes in Computer Science, Vol. 4818, pp. 259-266, Springer, BerlinHeidelberg-New York, 2008.

[64] R.H.W. Hoppe, C. Linsenmann, and S.I. Petrova. Primal-dual Newton methods in structural optimization. Comp. Visual. Sci., 9, 71-87, 2006

[65] R.H.W. Hoppe and S.I. Petrova. Primal-dual Newton interior-point methods in shape and topology optimization. Numer. Linear Algebra Appl., 11, 413-429, 2004.

[66] R.H.W. Hoppe, S.I. Petrova, and V. Schulz. A primal-dual Newtontype interior-point method for topology optimization. Journal of Optimization: Theory and Applications, 114, 545-571, 2002.

[67] Institute of Electrical and Electronics Engineers. Standard on piezoelectricity. IEEE Transactions on Sonics and Ultrasonics, 3, 1978.

[68] F. Ihlenburg. Finite Element Analysis of Acoustic Scattering. Springer, New York, 1998.

[69] H. Kardestuncer (ed.). Finite Element Handbook. McGraw-Hill, New York, 1987.

[70] A. Klawonn. An optimal preconditioner for a class of saddle point problems with a penalty term. SIAM J. Sci. Comput., 19, 540-552, 1998

[71] D. Köster. Numerical simulation of acoustic streaming on SAWdriven biochips. SIAM J. Comp. Sci., 29, 2352-2380, 2007.

[72] K. Kunisch and S. Volkwein. Galerkin proper orthogonal decomposition methods for parabolic problems. Numer. Math. 90, 117 148,2001

[73] K. Kunisch and S. Volkwein. Galerkin proper orthogonal decomposition methods for a general equation in fluid dynamics. SIAM J. Numer. Anal. 40, 492-515, 2002.

[74] K. Kunisch and S. Volkwein. Proper orthogonal decomposition for optimality systems. M2AN, 42, 1-23, 2008.

[75] S. Lall, J. E. Marsden, and S. Glavaški. A subspace approach to balanced truncation for model reduction of nonlinear control systems. Internat. J. Robust Nonlinear Control, 12, 519-535, 2002.

[76] R. Lerch. Simulation of piezoelectric devices by two- and threedimensional finite elements. IEEE Trans. Ultrasonics, Ferroelectrics and Frequency Control, 37, 233-247, 1990.

[77] J.-R. Li and J. White. Low rank solution of Lyapunov equations. SIAM J. Matrix Anal. Appl., 24, 260-280, 2002.
[78] W.G. Litvinov. Optimization in elliptic problems with applications to mechanics of deformable bodies and fluid mechanics. Birkhäuser, Basel, 2000

[79] G.A. Maugin. Continuum Mechanics of Electromagnetic Solids. North-Holland, Amsterdam, 1987

[80] V. Mehrmann and T. Stykel. Balanced truncation model reduction for large-scale systems in descriptor form. In: Dimension Reduction of Large-Scale Systems (P. Benner, V. Mehrmann, and D. C. Sorensen, eds.), pp. 83-115, Lecture Notes in Computational Science and Engineering, Vol. 45, Springer, Berlin-Heidelberg-New York, 2005.

[81] B. Mohammadi and O. Pironneau. Applied Shape Optimization for Fluids. Oxford University Press, Oxford, 2001

[82] B. C. Moore. Principal component analysis in linear systems: controllability, observability, and model reduction. IEEE Trans. Automat. Control, 26, 17-32, 1981.

[83] R.A. Nicolaides. Existence, uniqueness and approximation for generalized saddle point problems. SIAM J. Numer. Anal., 19, 349-357, 1982.

[84] J. Nocedal, A. Wächter, and R.A. Waltz. Adaptive barrier update strategies for nonlinear interior methods. Research Report RC 23563, IBM T. J. Watson Research Center, Yorktown, 2006.

[85] P. Oswald. Multilevel Finite Element Approximation: Theory and Applications. Teubner, Stuttgart, 1994.

[86] T. Penzl. Eigenvalue decay bounds for solutions of Lyapunov equations: the symmetric case. Syst. Control Lett., 40, 139-144, 2000.

[87] O. Pironneau. Optimal Shape Design for Elliptic Systems. Springer, Berlin-Heidelberg-New York, 1984.

[88] J. Pollard and B. Castrodale. Outlook for DNA microarrays: Emerging applications and insights on optimizing microarray studies. Report. Cambridge Health Institute, Cambridge, 2003.

[89] C. W. Rowley. Model reduction for fluids, using balanced proper orthogonal decomposition. Int. J. on Bifurcation and Chaos, 15, 997-1013, 2005.

[90] H.M. Shapiro. Practical flow cytometry. Wiley-Liss, New York, 2003.

[91] A.R. Shenoy, M. Heinkenschloss, and E.M. Cliff. Airfoil design by an all-at-once approach. Int. J. Comput. Fluid Mechanics, 11, 3-25, 1998.

[92] D. Silvester and A. Wathen. Fast iterative solution of stabilised Stokes systems part I: Using simple diagonal preconditioners. SIAM J. Numer. Anal., 30, 630-649, 1993.

[93] D. Silvester and A. Wathen. Fast iterative solution of stabilised Stokes systems part II: Using general block preconditioners. SIAM J. Numer. Anal., 31, 1352-1367, 1994.

[94] J. Sokolowski and J.P. Zolesio. Introduction to Shape Optimization. Springer, Berlin-Heidelberg-New York, 1992.

[95] L. Tartar. Introduction to Sobolev Spaces and Interpolation Theory. Springer, Berlin-Heidelberg-New York, 2007.

[96] A.L. Tits, A. Wächter, S. Bakhtiari, T.J. Urban, and C.T. Lawrence. A primal-dual interior-point method for nonlinear programming with strong global and local convergence properties. SIAM J. on Optimization, 14, 173-199, 2003.

[97] M. Ulbrich, S. Ulbrich, and L. Vicente. A globally convergent primal-dual interior point filter method for nonconvex nonlinear programming. Math. Programming, 100, 379-410, 2004.

[98] R.J. Vanderbei and D.F. Shanno. An interior point algorithm for nonconvex nonlinear programming. Computational Optimization and Applications, 13, 231-252, 1999

[99] P. Ventura, J.M. Hodé, J. Desbois, and M. Solal. Combined FEM and Green's function analysis of periodic SAW strucutre, application to the calculation of reflection and scattering parameters. IEEE Trans. Ultrasonics, Ferroelectrics and Frequency Control, 48, 1449-1461, 2001

[100] A. Wächter and L.T. Biegler. Line search filter methods for nonlinear programming: motivation and global convergence. SIAM J. on Optimization, 16, 1-31, 2005.

[101] G.M. Whitesides. The origins and the future of microfluidics. Nature, 44, 368, 2006

[102] K. Willcox and J. Peraire. Balanced model reduction via the proper orthogonal decomposition. AIAA 40, 2323-2330, 2002.

[103] G. Wittum. On the convergence of multigrid iterations with transforming smoothers. Theory with applications to the Navier-Stokes equations. Numer. Math., 57, 15-38, 1989.

[104] A. Wixforth. Acoustically driven programmable microfluidics for biological and chemical applications. JALA, 11, 399-405, 2006.

[105] A. Wixforth, J. Scriba, and G. Gauer. Flatland fluidics. Mst News, 5, 42-43, 2002 
[106] M.H. Wright. Interior methods for constrained optimization. Acta Numerica, 1, 341-407, 1992.

[107] S.J. Wright. Primal-Dual Interior-Point Methods. SIAM, Philadelphia, 1997.

[108] L.Y. Yeo and J.R. Friend. Ultrafast microfluidics using surface acoustic waves. Biomicrofluidics, 3, 012002-012023, 2009.

[109] E. Zeidler. Nonlinear Functional Analysis and Its Applications. II/A: Linear Monotone Operators. Springer, Berlin-HeidelbergNew York, 1990.

[110] J. Zelenka. Piezoelectric Resonators and Their Applications. Elsevier, Amsterdam, 1986

[111] K. Zhou, J. C. Doyle, and K. Glover. Robust and Optimal Control. Prentice Hall, Englewood Cliffs, 1996. 